$$
\begin{aligned}
& \text { UNIVERSIDADE DE SÃO PAULO } \\
& \text { INSTITUTO DE GEOCIENCIAS }
\end{aligned}
$$

\title{
AVALIAÇÃO DE DIFERENTES MEDIDAS DE SIMILARIDADE NO SIMDISPAT - NOVO ALGORITMO DE SIMULAÇÃO DE MÚLTIPLOS PONTOS
}

\section{KARLA XIMENA MORALES RODRIGUEZ}

Orientador: Prof. Dr. Marcelo Monteiro da Rocha

$$
\text { Dissertação de Mestrado }
$$

№ 871

COMISSÃO JULGADORA

Dr. Marcelo Monteiro da Rocha

Dra. Michelle Chaves Kuroda Avansi

Dr. Cleyton de Carvalho Carneiro

SÃO PAULO

2021 


\section{UNIVERSIDADE DE SÃO PAULO \\ INSTITUTO DE GEOCIENCIAS}

\section{AVALIAÇÃO DE DIFERENTES MEDIDAS DE SIMILARIDADE NO SIMDISPAT - NOVO ALGORITMO DE SIMULAÇÃO DE MÚLTIPLOS PONTOS}

KARLA XIMENA MORALES RODRIGUEZ

Dissertação apresentada ao programa de Geociências Recursos Minerais e Hidrogeologia para a obtenção do titulo de Mestre em Ciências

Área de concentração: Recursos Minerais e Meio Ambiente

Orientador: Prof. Dr. Marcelo Monteiro da Rocha 
Autorizo a reprodução e divulgação total ou parcial deste trabalho, por qualquer meio convencional ou eletrônico, para fins de estudo e pesquisa, desde que citada a fonte.

Serviço de Biblioteca e Documentaçäo do IGc/USP

Ficha catalográfica gerada automaticamente com dados fornecidos pelo(a) autor(a)

via programa desenvolvido pela Seçäo Técnica de Informática do ICMCNSPP

Bibliotecários responsáveis pela estrutura de catalogação da publicação: Sonia Regina Yole Guerra - CRB-8/4208 | Anderson de Santana - CRB-8/6858

Morales Rodriguez, Karla Ximena

AVALIACX̃O DE DIPRRENTES MEDIDAS DE SIMILARIDADB NO STMDISPAT NOVO ALGORITMO DE STMULACǨO DE

MÚLTIPLOS PONTOS / Karla Ximena Moralea Rodriguez; orientador Marcelo Monteiro da Rocha. -- Säo Paulo, 2021

$107 \mathrm{p}$

Dianertação (Mentrado - Programa de P6a-Graduação en Recurson Ninerain e Hidrogeologia) -- Instituto de Geociencias, Univeraidade de São Paulo, 2021.

1. Imagem de Treinamento. 2. Template. 3. Banco de dadoa de Padröen. 4. Batatiatican de mliltiplon ponton (MPS) - 5. Punç̄̃o de conectividade. I.

Monteiro da Rocha, Narcelo, orient. II. Titulo. 


\section{AGRADECIMENTOS}

Quero começar por agradecer ao meu orientador, Professor Marcelo Monteiro da Rocha, que me deu seu apoio desde o primeiro dia, seu tempo, seus conselhos e compartilhou comigo sua sabedoria e experiência, os quais foram imprescindíveis para a conclusão deste trabalho. Agradeço também pela paciência, dedicação e pelo tempo livre que compartilhou com todos seus alunos na sala 105 .

Ao mesmo tempo, agradeço ao instituto de geociências pela formação acadêmica recebida e à universidade de são Paulo por todos os benefícios adquiridos e por permitir-me pertencer a uma universidade tão prestigiosa.

À CAPES pelo auxílio concedido na forma de bolsa de estudos durantes estes dois anos de pesquisa.

Aos meus pais que são o pilar da minha vida, meu pai Julio que sempre com sua tranquilidade, amor e trabalho incansável pela família me ensinou a ser persistente em qualquer circunstância da vida. Minha mãe Mery, uma mulher de caráter tranquilo e sincero, me ensinou a amar e respeitar o próximo, um exemplo de mãe e de mulher que, independentemente das circunstâncias da vida, são sempre seus filhos em primeiro lugar.

Ao meu esposo Santiago, companheiro, amigo, cúmplice que está sempre ao meu lado fornecendo-me apoio, dedicação, amor e respeito. Obrigada por me acompanhar nessa etapa da minha vida, pelos dias e noites de estudo que compartilhamos, por estar nesses dias de angústia e estresse. Você é parte dessa conquista.

A meu irmão Cesar e minha irmã Diana, que me ensinaram o valor do trabalho árduo, a amizade, e de a unidade entre irmãos. E finalmente meus seis sobrinhos que sempre me deixam feliz cada vez que brinco com eles. 


\section{RESUMO}

As técnicas Geoestatísticas implementadas na modelagem de fenômenos espaciais, inicialmente foram baseadas em funções de probabilidades construídas a partir da estatística de dois pontos. No entanto, durante o decorrer dos anos, diferentes limitações foram encontradas, principalmente na reprodução de estruturas e características geológicas complexas. Na procura de uma solução, diferentes pesquisadores usaram estatísticas de mais do que dois pontos, denominada de simulação de multipontos (MPS). MPS emprega o conceito de Imagem de Treinamento (TI), que é um modelo geológico conceitual, que representa a continuidade espacial. O objetivo deste trabalho é desenvolver um novo algoritmo de simulação baseado nas MPS. O programa proposto é denominado SIMDISPAT, construído com base em conceitos do algoritmo do SNESIM (Single Normal Equations Simulation) e em conceitos do algoritmo do SIMPAT (Simulation with Patterns). O algoritmo foi escrito na linguagem de programação R. O método SIMDISPAT (Simulations with Distance and Pattern) simula imagens com características geológicas complexas, seja em $2 D$ ou $3 D$. Para verificar a eficiência do algoritmo, são utilizados quatro bancos de dados sintéticos descritos na literatura (três bidimensionais e um tridimensional). Além disso, quatro distâncias de similaridade são testadas, duas amplamente aplicadas nos MPS - a distância de Manhattan e a distância Euclidiana - as outras duas distancias são comuns em diferentes áreas das ciências - a distância de Lorentz e a distância de Cosseno. Para comparar os resultados obtidos pelo algoritmo, utilizou-se a análise de conectividade para verificar qual das distâncias reproduz melhor as características de cada uma das $\boldsymbol{T I}$, além disso, foi utilizado um método visual denominado escalonamento multidimensional (MDS) para explorar a estrutura de dados de similaridade. O SIMDISPAT é capaz de efetivamente reproduzir efetivamente as diferentes características das $\boldsymbol{T I}$, com as distâncias de Manhattan, Euclidiana e Lorentz, porém à distância Cosseno apresenta problemas ao reproduzir os padrões das $\boldsymbol{T I}$. Destaca-se que a distância Lorentz, que não é utilizada no MPS, reproduz satisfatoriamente as diferentes características das $\boldsymbol{T I}$. 


\section{ABSTRACT}

The Geostatistics techniques implemented in the modeling of spatial phenomena were initially based on probability functions built from two-point statistics. However, over the years, different limitations were found, mainly in the reproduction of structures and complex geological features. In search of a solution, different researchers used statistics of more than two points, called multipoint simulation (MPS). MPS employs the concept of Training Image (TI), which is a conceptual geological model that represents spatial continuity. The objective of this work is to develop a new simulation algorithm based on MPS. The proposed program is called SIMDISPAT, based on concepts from the SNESIM algorithm (Single Normal Equations Simulation) and on concepts from the SIMPAT algorithm (Simulation with Patterns). The algorithm was written in the $R$ programming language. The SIMDISPAT (Simulations with Distance and Pattern) method simulates images with complex geological characteristics, either in $\mathbf{2 D}$ or $\mathbf{3 D}$. To verify the algorithm's efficiency, four synthetic databases described in the literature are used (three two-dimensional and one threedimensional). In addition, four similarity distances are tested, two widely applied in MPS - the Manhattan distance and the Euclidean distance - the other two distances are common in different areas of the sciences - the Lorentz distance and the Cosine distance. To compare the results obtained by the algorithm, connectivity analysis was used to verify which of the distances best reproduces the characteristics of each $\boldsymbol{T I}$, in addition, a visual method called multidimensional scaling (MDS) was used to explore the structure of similarity data. SIMDISPAT is able to effectively reproduce the different characteristics of the $\boldsymbol{T I}$, with the distances of Manhattan, Euclidean and Lorentz, but at the Coseno distance it presents problems when reproducing the TI patterns. It is noteworthy that the Lorentz distance, which is not used in the MPS, satisfactorily reproduces the different characteristics of $\boldsymbol{T I}$. 


\section{SUMARIO}

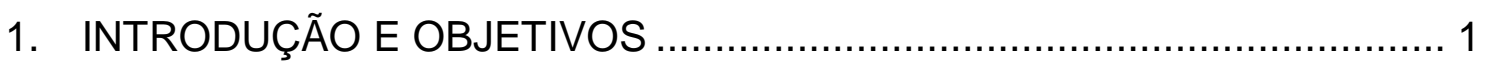



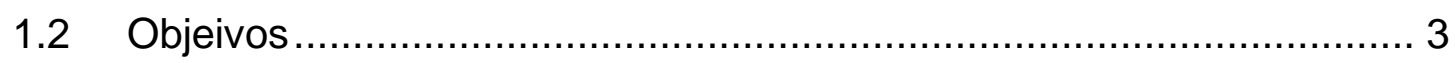

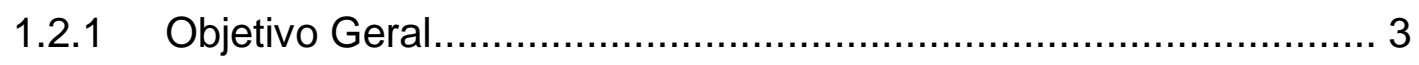

1.2.2 Objetivos Específicos ........................................................... 3

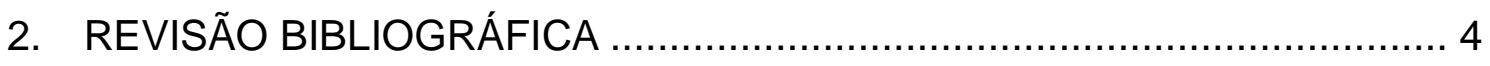

2.1 Imagem de Treinamento .......................................................... 9

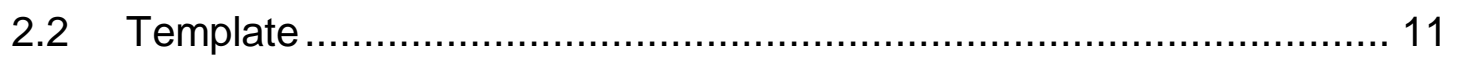

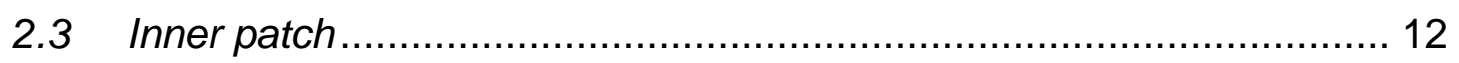





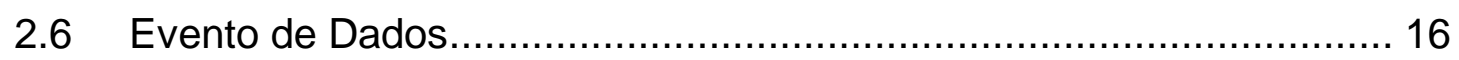



2.7.1 Distância de Manhattan ....................................................... 18

2.7.2 Distância Euclidiana .................................................................. 19

2.7.3 Distância Lorentz ................................................................. 21

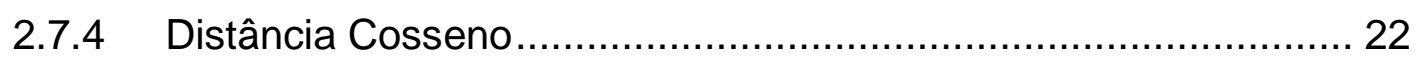

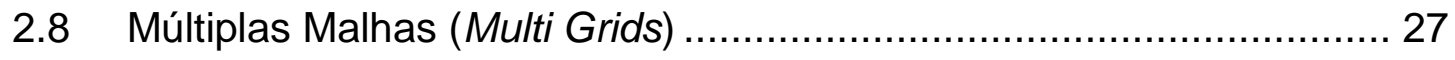

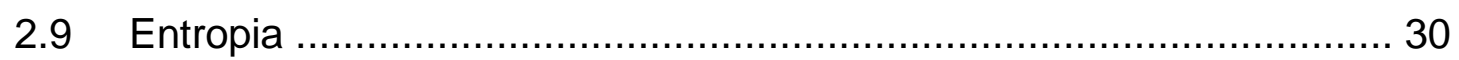

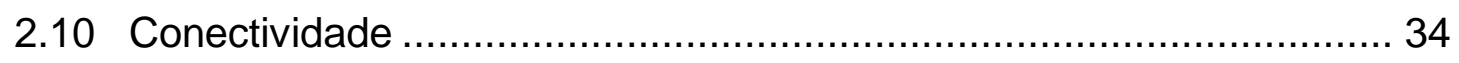

2.10.1 Descrição da Função CONNEC3D .......................................... 34

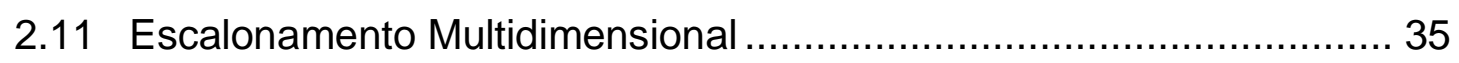









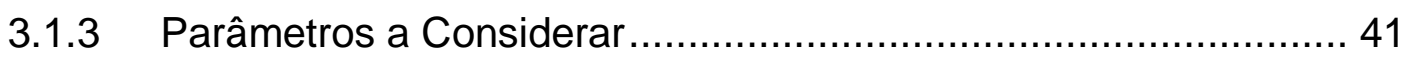

3.1.4 Parâmetros da Conectividade.................................................... 44



4. APRESENTAÇÃO DOS RESULTADOS …………............................. 49




4.2 Modelo de Canal Binário Bidimensional ......................................... 49

4.3 MODELO CATEGÓRICO DE QUATRO CATEGORIAS EM 2D ......... 52

4.4 Modelo Contínuo Bidimensional ........................................................ 56



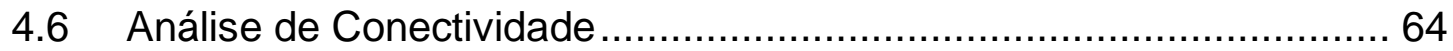

4.7 Análise por Escalonamento Multidimensional (MDS) ….................... 67

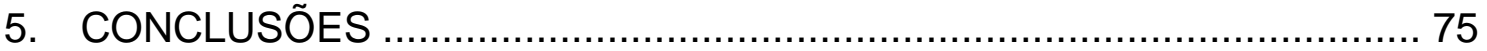

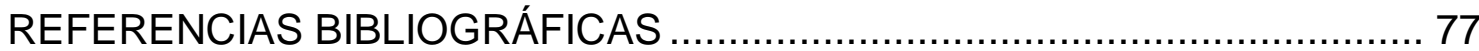



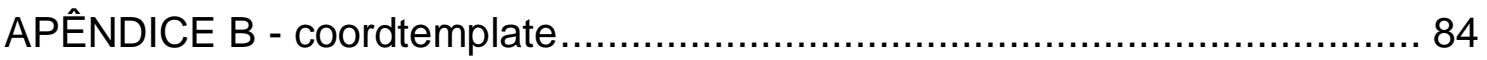

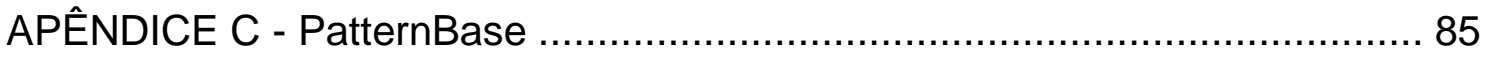

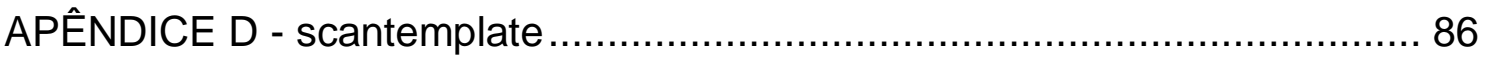













APÊNDICE K - pastepattern ............................................................... 92 


\section{LISTA DE FIGURAS}

Figura 1.1 Complexidade geológica. $\boldsymbol{A}$ ) Alvuial Leque, $\boldsymbol{B}$ ) Rio meandrante, $\boldsymbol{c}$ ) Sistema Deltaico e $\boldsymbol{D}$ ) Recife de Coral (Fonte: CAERS, 2011)....................... 2

Figura 2.1 Árvore de busca criada pela varredura de uma imagem de treinamento (TI) binária, que armazena as probabilidades condicionadas derivadas das respectivas estruturas, capturadas pelo template T. (Fonte:

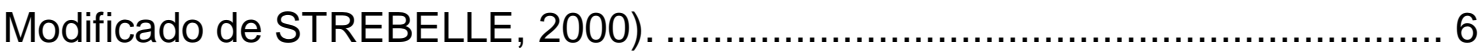

Figura 2.2 Conjunto de filtros (Fonte: modificada de ZHANG 2006) ................. 7 Figura 2.3 Transformação de kernel. (FONTE: HONARKHAH, E CAERS 2010).

\section{8}

Figura 2.4 Regiões de sobreposição são mostradas pela cor cinza sombreada (Fonte: TAHMASEBI et al. 2012).

Figura 2.5 Exemplos de imagens de treinamento para simulações bidimensional e tridimensional. a) dados exaustivos de Walker Lake categorizados; b) Modelagem não estacionária; c) Malha 3D que representa as hidrofácies em um aqüífero aluvial no vale de Maules Creek, Austrália. (Fonte: MARIETHOZ E CAERS, 2014). ............................................................ 11

Figura 2.6 Representação de um Template bidimensional $\mathbf{5} \times \mathbf{5}$. O vetor $\mathbf{h 1}=\mathbf{0}$ identifica a localização central $\boldsymbol{u}$ do $\boldsymbol{T}$ em uma malha com dimensões $\mathbf{1 1} \times \mathbf{1 1}$

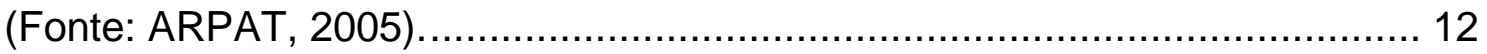

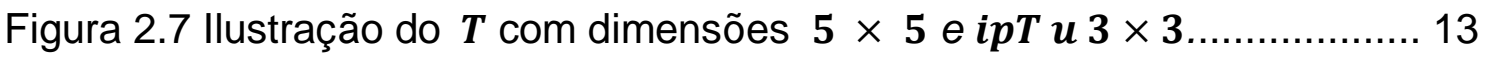

Figura 2.8 Varredura da imagem de treinamento para a construção do banco de dados de padrões através do $T$ bidimensional de tamanho 3x3. (Fonte:

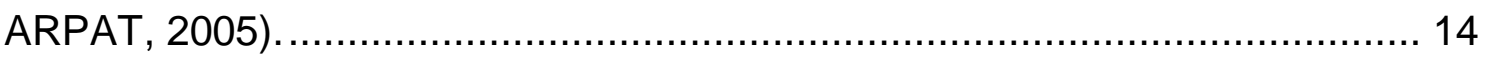

Figura 2.9 Cálculo de similaridade de padrões binários, aplicando a distância de Manhattan (Fonte: ARPAT, 2005)...................................................... 19

Figura 2.10 Cálculo da similaridade de padrões binários, fazendo uso da

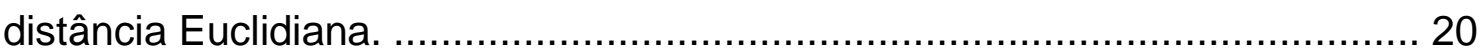

Figura 2.11 Cálculo de similaridade de padrões binários, aplicando a distância

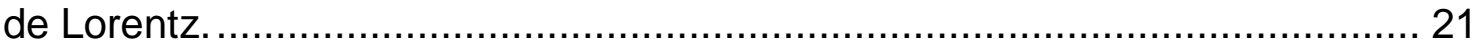

Figura 2.12 Distância Cosseno, utilizando a amostras de padrões binários. ... 23

Figura 2.13 Caminho aleatório de todos os nós do Gre................................ 24

Figura 2.14 Passo a passo do algoritmo, usando a TI e o banco de dados de padrões da Figura 2.8 e um template $\mathbf{5} \times \mathbf{5}$, além disso, são utilizados dados amostrados em uma área de estudo .................................................... 26

Figura 2.15 Continuação da Figura 2.14. Aplicando o algoritmo passo a passo até que uma realização seja obtida......................................................... 27 Figura 2.16 llustração de uma $\boldsymbol{T I}$, usando três multi-grids de tamanho $\mathbf{5} \times \mathbf{5 . 2 9}$ 
Figura 2.17 Imagem de treinamento 2D, representando canais sinuosos (Fonte: MARIETHOZ E CAERS, 2014) ............................................................ 32 Figura 2.18 A curva de entropia média (Fig. 2.18a) e a probabilidade de registro do perfil que indica que o melhor template possui um tamanho de $13 \times 13$ (Fig.2.18b) 33

Figura 3.1 Imagens de treinamento. (a) Imagem de treinamento do canal (STREBELLE 2002), (b) TI de uma variável categórica, deposição de rios com quatro categorias (HONARKHAH \& CAERS, 2010), (c) Imagem de treinamento de muro de pedra (ZHANG, 2006)...................................................... 37

Figura 3.2 TI binaria em 3D (Fonte: HONARKHAH \& CAERS, 2010)............ 37

Figura 3.3 Mapa de localização das amostras selecionadas pelo método da amostragem simples (porcentagem de 1\%). (a) Primeira amostra do Banco de dados do canal (b) Segunda amostra do banco de dados de deposição fluvial com quatro categorias e (c) Terceira amostra do banco de dados de muro de pedra

Figura 3.4 Distribuição espacial dos dados condicionantes pelo método de amostragem simples, para o banco de dados em 3D ................................ 40

Figura 3.5 A entropia de Shannon gera o tamanho do ótimo do template para cada banco de dados 2D. A Figura mostra a TI (esquerda), a curva de entropia média (meio) e o perfil de probabilidade (direita). ......................................... 43 Figura 3.6 Resultado da função CoordTemplate. Valores iguais a um representam os nós congelados durante a simulação.................................. 45 Figura 3.7 Obtenção de padrões, através de amostragem simples, para a construção do banco de dados a partir de um template de tamanho $\mathbf{5} \times \mathbf{5}$ (Fonte: modificado de ARPAT, 2005)................................................... 46

Figura 3.8 Fluxograma do algoritmo SIMDISPAT ..................................... 48

Figura 3.9 Representação do método de análise de conectividade................. 48

Figura 4.1 Resultados das simulações obtidas pelo algoritmo SIMDISPAT, para cada uma das quatro distâncias propostas, aplicado ao BD1 $(\boldsymbol{a}),(\boldsymbol{b})$ e (c) são

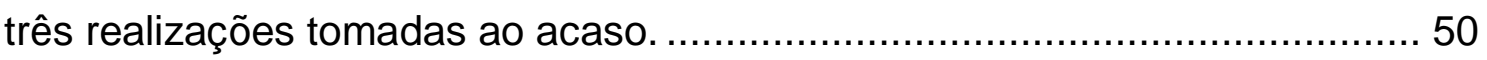
Figura 4.2 Os dados de condicionamento para o modelo do canal binário (à esquerda). O E-type das cem simulações geradas com 0 algoritmo SIMDISPAT, para cada uma das quatro distâncias (à direita)........................ 52 Figura 4.3 Resultados das simulações obtidas pelo algoritmo SIMDISPAT, para cada uma das quatro distâncias propostas, aplicado ao BD2 ......................... 54 Figura 4.4 Os dados de condicionamento para o modelo de quatro categorias (à esquerda). O E-type das 100 simulações geradas com o algoritmo SIMDISPAT, para cada uma das 4 distâncias selecionadas (à direita). .......... 56 Figura 4.5 Resultados das simulações obtidas pelo algoritmo SIMDISPAT, para cada uma das quatro distâncias propostas, aplicado ao BD3. 58 
Figura 4.6 Os dados de condicionamento para o modelo contínuo bidimensional (à esquerda). É observada o E-type das cem simulações geradas com o algoritmo SIMDISPAT, para cada uma das 4 distâncias selecionadas (à direita) 60

Figura 4.7 Resultados das simulações obtidas pelo algoritmo SIMDISPAT, para cada uma das três distâncias, aplicada ao BD4.

Figura 4.8 E-type das cem simulações geradas com o algoritmo SIMDISPAT, para cada uma das três distâncias selecionadas (BD4). 63

Figura 4.9 Função de conectividade para as realizações geradas por SIMDISPAT. As curvas coloridas tracejadas representam o resultado obtido para cada uma das cem simulações calculadas com cada uma das distâncias e a curva sólida preta é a conectividade obtida na $\boldsymbol{T I}$. 65 Figura 4.10 A função de conectividade calculada para as realizações geradas da figura 4.3. As curvas coloridas representam as realizações de cada uma das distâncias e a TI é mostrado de cor preto. 66 Figura 4.11 A função de conectividade calculada para as realizações geradas. 66

Figura 4.12 a) Representação do mapa de MDS, para demonstrar a variabilidade espacial de cada uma das simulações geradas pelo modelo binário $\mathbf{2 D}$. Como pode ser visto, a $\boldsymbol{D C}$ é azul, a $\boldsymbol{D E}$ é de cor verde, a $\boldsymbol{D} \boldsymbol{L}$ tem cor vermelha e em amarelo a $\boldsymbol{D} \boldsymbol{M}$. Por último a $\boldsymbol{T I}$ é representada por um ponto de cor preta. $\boldsymbol{b}$ ) A média do MDS 68

Figura 4.13 a) O gráfico MDS para demonstrar a variabilidade nas realizações geradas pelo $\boldsymbol{B D 2}$. $\boldsymbol{b}$ ) A média do MDS. 70 Figura 4.14 a) Representação do mapa MDS, para demonstrar a variabilidade espacial de cada uma das realizações geradas pelo modelo continuo.b) A média do MDS. ......................................................................... 72

Figura 4.15 a) Um mapa MDS para demonstrar a variabilidade espacial de cada uma das realizações geradas pelo modelo binário $3 \boldsymbol{D}$. b) A média do MDS. 74 


\section{LISTA DE TABLAS}

Tabela 3.1 Proporção do tipo de litologia a partir da $\boldsymbol{T I}$ do canal (Primeira $\boldsymbol{T I}$ ).

Tabela 3.2 Proporção do tipo de litologia a partir da $\boldsymbol{T I}$ do canal (Segunda $\boldsymbol{T I}$ ). 38

Tabela 3.3 Resumo estatístico da terceira TI do muro de pedra (Terceira $\boldsymbol{T I}$ ). 38

Tabela 3.4 Proporção do tipo de litologia a partir da TI em 3D. 38

Tabela 3.5 Proporção do tipo de litologia a partir dos dados amostrados (Primeira amostra do Banco de dados do canal binário 2D). 40

Tabela 3.6 Proporção do tipo de litologia a partir dos dados amostrados (segunda amostra do banco de dados). 40

Tabela 3.7 Resumo das estatísticas descritivas da terceira amostra do banco

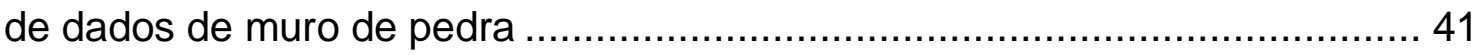
Tabela 3.8 Proporção do tipo de litologia a partir do banco de dados em 3D.. 41 Tabela 3.9 Parâmetros usados no algoritmo SIMDISPAT, para gerar as

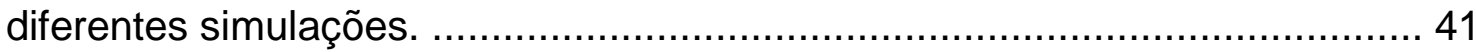
Tabela 3.10 Parâmetros utilizados na função CONNEC3D para a análises de



Tabela 4.1 Proporção do tipo de litologia para as simulações selecionadas DL. 51

Tabela 4.2 Proporção do tipo de litologia para as simulações selecionadas DE. 51

Tabela 4.3 Proporção do tipo de litologia para as simulações selecionadas DM. 51

Tabela 4.4 Proporção do tipo de litologia para as simulações selecionadas DC. 51

Tabela 4.5 Proporção do tipo de litologia para as simulações selecionadas DL. 55

Tabela 4.6 Proporção do tipo de litologia para as simulações selecionadas DE. 55

Tabela 4.7 Proporção do tipo de litologia para as simulações selecionadas DM. 55

Tabela 4.8 Proporção do tipo de litologia para as simulações selecionadas DC. 56

Tabela 4.9 Estatísticas descritivas para as realizações selecionadas da Figura

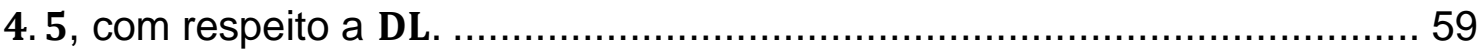


Tabela 4.10 Estatísticas descritivas para as realizações selecionadas da Figura 4.5, com respeito à $\mathbf{D E}$. 59

Tabela 4.11 Estatísticas descritivas para as realizações selecionadas da Figura 4. 5, com respeito a DM. 59

Tabela 4.12 Estatísticas descritivas para as realizações selecionadas da Figura 4. 5, com respeito a DC. 59

Tabela 4.13 Proporção do tipo de litologia para as simulações selecionadas DL. 62

Tabela 4.14 Proporção do tipo de litologia para as simulações selecionadas DE 62

Tabela 4.15 Proporção do tipo de litologia para as simulações selecionadas DM. 62 


\section{LISTA DE QUADROS}

Quadro 2.1 Descrição das funções PatterBase e ScanTemplate. ................... 15

Quadro 2.2 Descrição da função getDataEvent. ....................................... 24

Quadro 2.3 Descrição das funções similarPattern, disDistance e pastePattern.

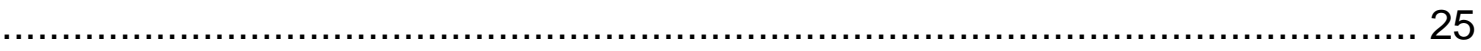

Quadro 2.4 Resumo da aplicação do método multi-grid............................... 29

Quadro 2.5 O algoritmo de entropia de Shannon para obter o $\boldsymbol{T}$ "ótimo"......... 32

Quadro 2.6 Dados de entrada para a função CONNEC3D. ......................... 35

Quadro 3.1 Descrição da função CoordTemplate do algortimo SIMDISPAT... 45

Quadro 3.2 Descrição da função PatternBase do algoritmo SIMDISPAT....... 47 


\section{LISTA DE SÍMBOLOS}

\begin{tabular}{|c|c|}
\hline SÍMBOLO & SIGNIFICADO \\
\hline $\bar{u}$ & Coordenada espacial $(x, y$ e $z)$ \\
\hline$T I$ & Imagem de treinamento \\
\hline $\operatorname{ti}(u)$ & Valor da imagem de treinamento no ponto $u$ \\
\hline$t i_{T}(u)$ & Vetor de múltiplos pontos dentro de um template \\
\hline$T$ & Template ou janela de procura \\
\hline$i p_{T}(u)$ & Inner patch \\
\hline$n_{T}$ & Tamanho do template \\
\hline $\boldsymbol{n}_{\text {pat }}$ & Número total de padrões \\
\hline $\boldsymbol{h}$ & Vetor distância \\
\hline $\operatorname{patd}_{T}$ & Base de dados de padrões \\
\hline $\boldsymbol{p a t}_{T}^{k}$ & Vetor único de padrões \\
\hline dbtol & Tolerância da partição do banco de dados \\
\hline patpercent & Porcentagem do banco de dados \\
\hline$G_{t i}$ & Malha regular cartesiana \\
\hline $\boldsymbol{r e}(\boldsymbol{u})$ & n-ésima realização \\
\hline $\operatorname{dev}_{T}(u)$ & $\begin{array}{l}\text { Vetor de sequência (dados amostrais e nós previamente } \\
\text { simulados) }\end{array}$ \\
\hline$w_{i}$ & Pesos relacionados a cada tipo de evento de dados \\
\hline$G_{r e}$ & Malha regular cartesiana da realização $\boldsymbol{r} \boldsymbol{e}$ \\
\hline$d<$ & $\begin{array}{l}\text { Função distancia (distância euclidiana, Manhattan, Lorentz ou } \\
\text { Cosseno) }\end{array}$ \\
\hline$s<$. $>$ & Função de similaridade \\
\hline$G^{g}$ & Malha cascata \\
\hline$T^{g}$ & Template grosso \\
\hline
\end{tabular}




\section{INTRODUÇÃO E OBJETIVOS}

\subsection{Introdução}

A simulação estocástica geoestatística ganhou popularidade como uma ferramenta quantitativa para gerar vários modelos, que honram determinada estrutura estatística e vários tipos de dados, medidos ou interpretados, na reprodução de variáveis regionalizadas contínuas ou categóricas. A simulação estocástica visa criar múltiplas representações realistas, chamadas realizações, do fenômeno espacial em estudo, que são equiprováveis (DEUTSCH \& JOURNEL, 1992; OLEA, 1999; CHILÈS \& DELFINER, 1999). A simulação estocástica é dita condicional se os resultados das realizações honrarem os pontos amostrais nos respectivos locais (DEUTSCH \& JOURNEL, 1992).

Existem duas abordagens importantes tradicionalmente utilizadas na simulação de um atributo não amostrado: a primeira é baseada em células, por exemplo: simulação sequencial gaussiana (SGS), simulação sequencial indicadora (SIS), simulação por bandas rotativas entre outras, fundamentadas na correlação espacial representada pelo variograma (DEUTSCH \& JOURNEL, 1992; GOOVAERTS, 1997; CHILÈS \& DELFINER, 1999; OLEA, 1999). A segunda abordagem é baseada em objetos, subdivididos em duas classes: modelos Booleanos e modelos de pontos de Poisson (HALDORSEN \& CHANG, 1986; DEUTSCH \& WANG, 1996; CHILÈS \& DELFINER, 1999). Ambas as aproximações, apesar de serem amplamente aplicadas, na prática têm limitações na reprodução de modelos geológicos realistas, especialmente a modelagem baseada em células, uma vez que ela não consegue reproduzir complexidades geológicas como as exemplificadas na (Figura 1.1), ou seja, geometrias curvilíneas presentes em reservatórios fluviais, em razão de serem limitados à estatística de dois pontos (GUARDIANO \& SRIVASTAVA, 1993; STREBELLE, 2002; ARPAT \& CAERS, 2007). 
A simulação espacial da continuidade de um fenômeno pode ser realizada através de técnicas que usam as estatísticas de ordem da distribuição de múltiplos pontos (GUARDIANO \& SRIVASTAVA, 1993 e STREBELLE, 2000), dado que conseguem reproduzir padrões geológicos complexos (por exemplo, canais fluvias). A simulação estatística de múltiplos pontos emprega o conceito de imagem de treinamento (TI) (GUARDIANO \& SRIVASTAVA, 1993, STREBELLE, 2000; ARPAT \& CAERS, 2007). A imagem de treinamento é um modelo geológico conceitual, que representa a continuidade espacial do fenômeno explicitamente, em 2D ou 3D (ARPAT \& CAERS, 2007). Nesse sentido, a TI reflete as diferentes heterogeneidades da área ou depósito e pode vir de dados exaustivos, bem como de afloramentos, que são representativos da área em estudo. Além disso, um único resultado da simulação baseada em objetos, não condicional, é uma alternativa para obter uma imagem de treinamento (ARPAT \& CAERS, 2007).

A)



C)

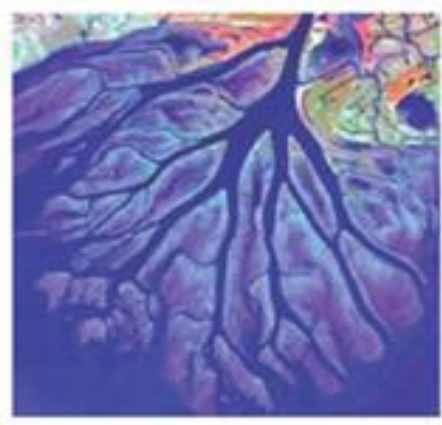

B)

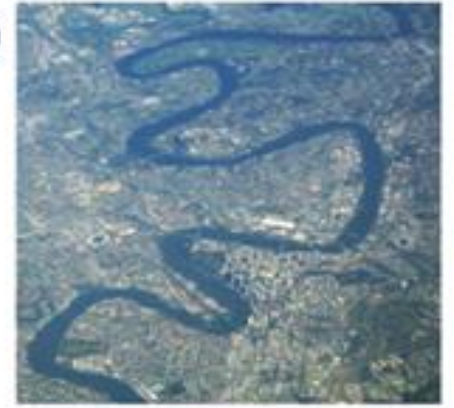

D)

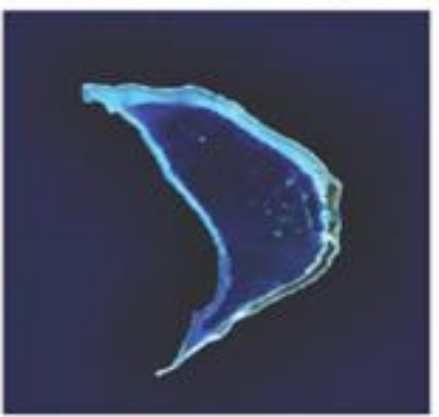

Figura 1.1 Complexidade geológica. A) Alvuial Leque, B) Rio meandrante, $\boldsymbol{c}$ ) Sistema Deltaico e D) Recife de Coral (Fonte: CAERS, 2011).

Strebelle (2002) propõe o primeiro algoritmo de simulação por múltiplos pontos computacionalmente práticos na geoestatística denominado SNESIM (Single Normal Equation Simulation), baseado na ideia original de Guardiano \& Srivastava (1993). A técnica de simulação de múltiplos pontos desenvolvida por 
Strebelle é um algoritmo sequencial, não iterativo, que armazena em uma árvore de busca as probabilidades condicionais calculadas por meio de uma única varredura da imagem de treinamento por um template pré-definido. Neste método cada ponto não amostrado é visitado uma única vez, estimado pelas equações normais simples e, finalmente, atribuído a nó da grid a partir da cdf pelo método de simulação de Monte Carlo. Neste trabalho é apresentado um algoritmo denominado SIMDISPAT, que foi utilizado para modelar padrões complexos em $\mathbf{2 D}$ e $\mathbf{3 D}$, fundamentados no reconhecimento de padrões presentes na $\boldsymbol{T I}$, onde foram utilizados quatro distâncias de similaridade para 0 relacionamento dos padrões.

\subsection{Objeivos}

\subsubsection{Objetivo Geral}

O objetivo geral deste trabalho é propor e desenvolver um algoritmo computacional, para a modelagem dos métodos de múltiplos pontos, que permita simular sistemas de deposição bidimensionais e tridimensionais.

\subsubsection{Objetivos Específicos}

- Avaliar a utilização de diferentes distâncias de similaridade para o reconhecimento de padrões, presentes na imagem de treinamento, para a simulação.

- Comparar os resultados das simulações obtidas do algoritmo SIMDISPAT, usando análise de conectividade e um escalonamento multidimensional (MDS), uma análise visual, permitindo interpretar e selecionar qual distância reproduz melhor o fenômeno. 


\section{REVISÃO BIBLIOGRÁFICA}

Esta seção descreve os conceitos fundamentais da teoria da estatística de múltiplos pontos.

De acordo com, Tahmasebi et al. (2012) as aplicações geoestatísticas são baseadas em estimadores lineares e modelos paramétricos. Métodos tradicionais, como a simulação sequência indicadora-SIS baseado em células, bem como o modelo baseado em objetos-OBM, apresentam aspectos inconvenientes diferentes: o primeiro por sua incapacidade para reproduzir características complexas e curvilíneas e o segundo tem a limitação de apresentar dificuldade em honrar os dados condicionais vindo de poços ou de furos de sonda.

Devido a essas limitações surgiu a necessidade do desenvolvimento de técnicas capazes de aproximar maiores complexidades. Desta forma, surge simulação de múltiplos pontos (MPS), que segundo Boucher (2011) foi inicialmente desenvolvida na Universidade de Stanford. A simulação de múltiplos pontos, fundamentada no conceito de imagens de treinamento, combina a habilidade de modelos baseados em objetos (para reproduzir formas) com a capacidade da simulação indicadora de condicionar as realizações aos dados. A TI consiste em um modelo conceitual da estrutura das fácies, por exemplo, que dada sua densidade de informação, permite a determinação de estatísticas de alta ordem ou estatísticas de múltiplos pontos onde é possível a substituição do modelo paramétrico tradicional baseado em variogramas (TAHMASEBI et al., 2018).

Segundo Strebelle (2002), no final dos anos 80 e início da década 90 do século passado, desenvolveu-se o conceito de métodos MPS cujas primeiras bases foram consolidadas por Farmer (1988), Deutsch (1992) Guardiano e Sirivastava (1993), apresentaram pela primeira vez a teoria matemática dos métodos de múltiplos pontos e um algoritmo denominado Extended Normal Equation Simulation (ENESIM). A ENESIM consiste em uma SIS que usa uma ou várias imagens de treinamento (TI) (GUARDIANO \& SIRIVASTAVA, 1993). 
O método apresentou uma grande desvantagem computacional (grande demanda de CPU), uma vez que a TI deveria ser varrida cada vez que um novo nó não amostrado era visitado (STREBELLE, 2002)

O algoritmo proposto por Strebelle (2002) conhecido como SNESIM (Single Normal Equation Simulation) é um dos métodos probabilísticos mais eficientes para a simulação de múltiplos pontos. De acordo com Strebelle (2002), o primeiro passo no SNESIM é a definição de um template $\boldsymbol{T}$, que varra toda a imagem de treinamento definindo padrões que são armazenados em uma árvore de busca utilizada para calcular as probabilidades condicionais em cada ponto a simular, conforme ilustrado na Figura 2.1. Tahmasebi et al. (2012) destacam que a eficiência computacional do SNESIM fica comprometida quando geometrias complexas são usadas, por exemplo, multi-facies e/ou imagens tridimensionais ou quando o processo de simulação é aplicado a milhões de células. Porém para Strebelle \& Cavelius (2014), isso não significa que $o$ algoritmo não possa ser usado para simular modelos tridimensionais.

Métodos alternativos, para melhorar o algoritmo SNESIM, são propostos por diferentes autores baseados, principalmente no conceito de similaridade. Um dos primeiros algoritmos é apresentado por Arpat \& Caers (2004) denominado: SIMPAT (Stochastic simulation with Patterns). Esse método tem a capacidade de conservar a continuidade e configuração da imagem de treinamento (TAHMASEBI, 2018), dado que simula padrões, ou seja, conjuntos de pixels, em vez de simular pixel por pixel como no algoritmo SNESIM. Da mesma forma, o SIMPAT substitui as representações probabilísticas tradicionais usadas no SNESIM e esses ajustes reduzem o tempo de processamento além de melhorarem a continuidade dos padrões. No entanto, 0 SIMPAT mostra que o tempo de uso de CPU é muito prologando, principalmente, quando são utilizados em simulações $3 D$ (TAHMASEBI, 2018). 


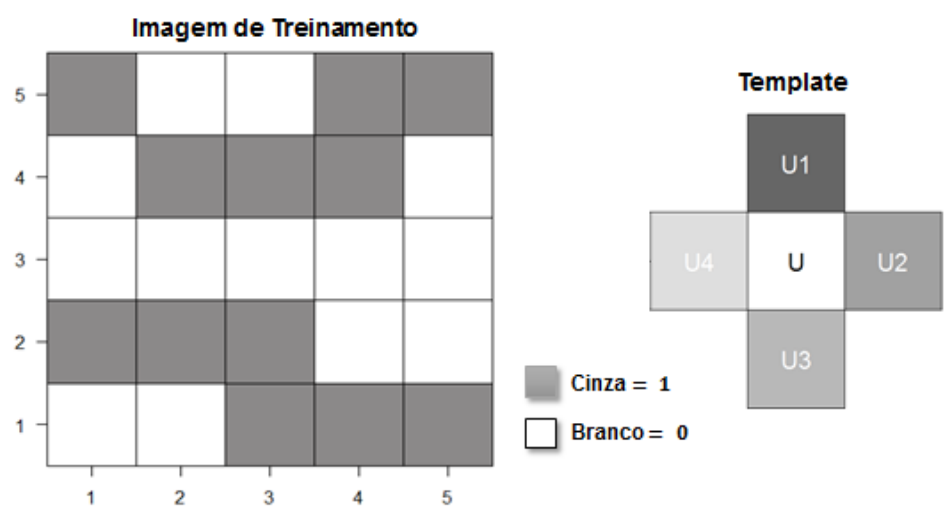

Árvore de Procura

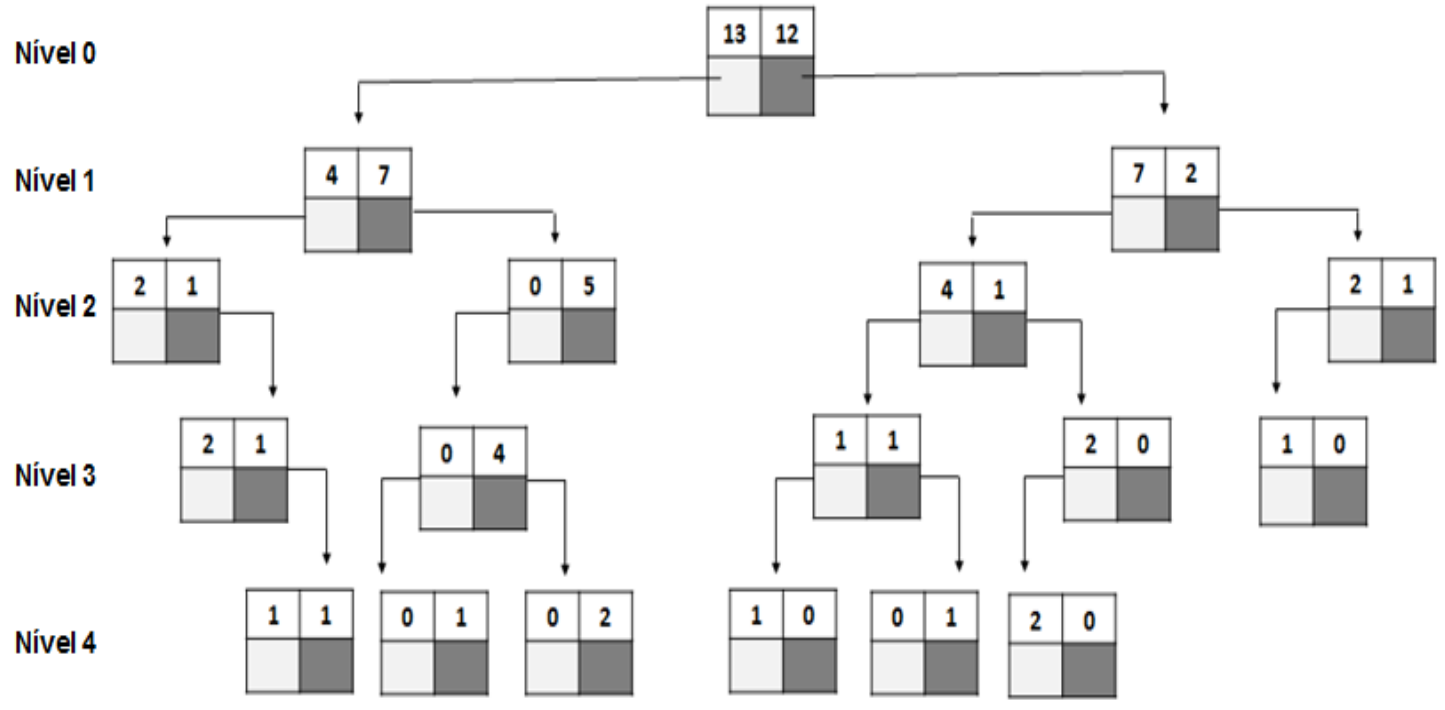

Figura 2.1 Árvore de busca criada pela varredura de uma imagem de treinamento (TI) binária, que armazena as probabilidades condicionadas derivadas das respectivas estruturas, capturadas pelo template T. (Fonte: Modificado de STREBELLE, 2000).

Zhang et al., (2006) propõem outro método baseado em padrões para contornar os problemas observados no SIMPAT. Denominado FILTERSIM (Filter-Based Simulation). A técnica usa filtros lineares (em 2D, 6 filtros, ver Figura 2.2), para classificar padrões da imagem de treinamento, reduzindo suas dimensões, para assim, convertê-los em um pequeno conjunto de pontuações de filtro (score space) (REMY et al., 2009). Essa conversão acelera o processo de busca e reduz o tempo computacional, dado o número limitado de filtros de execução, em comparação com SIMPAT. Sua aplicação é possível tanto para variáveis discretas quanto para variáveis contínuas. Deve-se considerar que a seleção de filtros apropriados depende do usuário, o que resulta em um problema da técnica FILTERSIM (TAHMASEBI, 2018). 

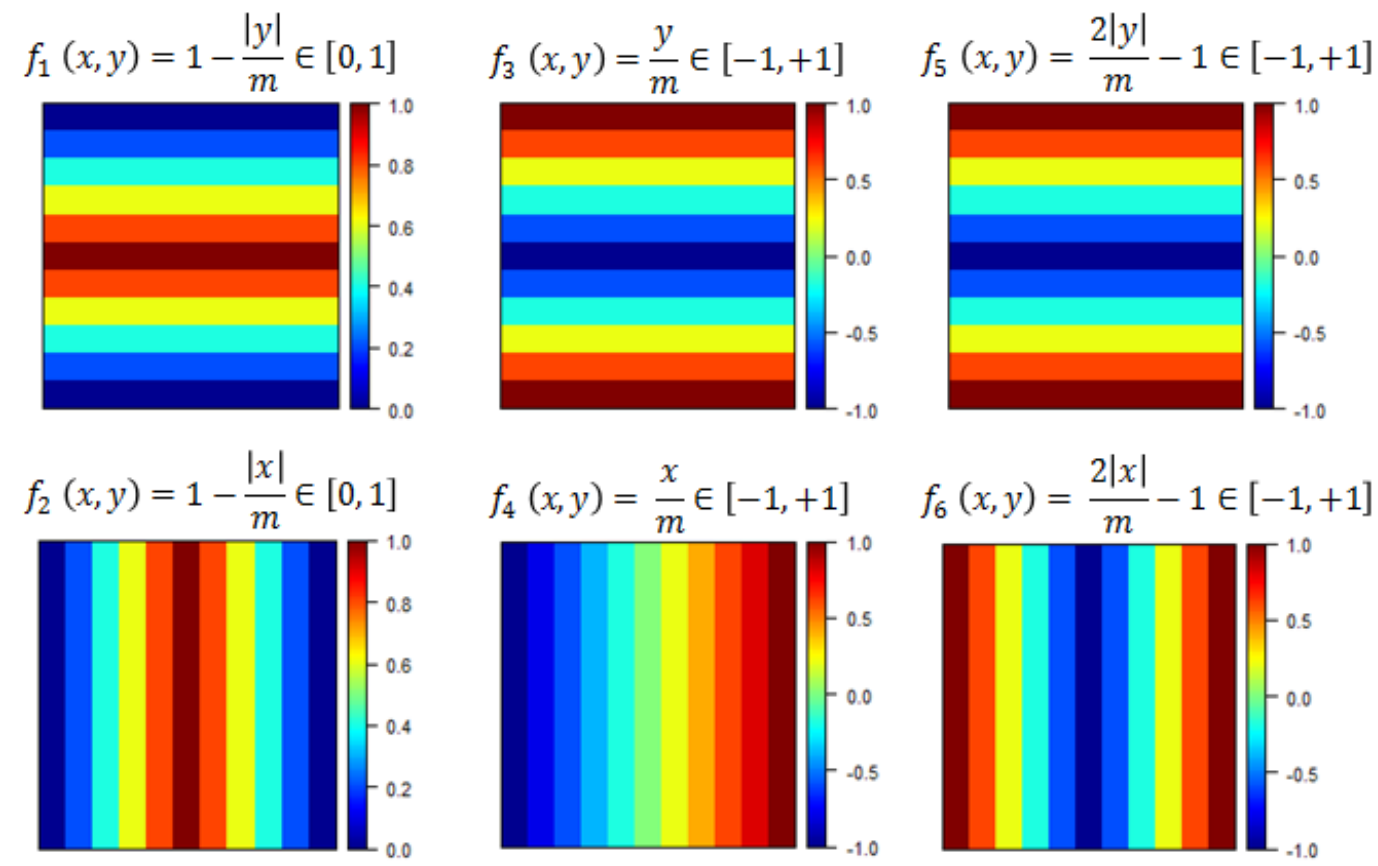

Figura 2.2 Conjunto de filtros (Fonte: modificada de ZHANG 2006).

Honarkhah \& Caers (2010) propuseram um algoritmo de simulação de padrões, baseado na distância e o mapeamento do Kernel chamado DISPAT (Distance- based Pattern modeling) (Figura 2.3). O algoritmo DISPAT é similar ao FILTERSIM, exceto que os filtros usados para classificação de padrões são substituídos por um núcleo $k$-means aplicado em uma transformação MDS dos padrões de TI (HONARKHAH \& CAERS, 2010). Esta técnica usa um método de escalonamento multidimensional (MDS), que reduz a dimensão dos padrões (espaço cartesiano), para aplicar a transformação do Kernel, com o objetivo de diferenciar e agrupar por K-means, da melhor maneira possível, os padrões espaciais ou espaço de características (HONARKHAH \& CAERS, 2010). 

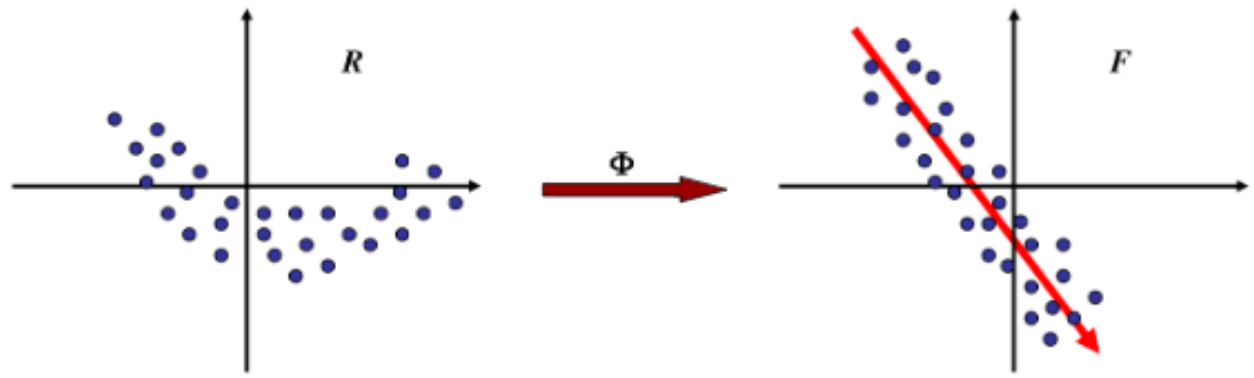

Figura 2.3 Transformação de kernel. (FONTE: HONARKHAH, E CAERS 2010).

Outro método de simulação baseado em padrões, introduzida por Tahmasebi et al. (2012), é a simulação baseada em correlação cruzada ao longo de um caminho de varredura (CCSIM Cross-Correlation based Simulation). Computacionalmente, este método é mais eficiente do que os métodos de MPS anteriores, uma vez que, o algoritmo não avança por padrões completos, mas ponto por ponto. Em contraste com os métodos de multiponto que usam eventos de dados de maior dimensão na identificação do padrão mais similar, o CCSIM utiliza o conceito de superposição, que acaba sendo menor que o evento de dados (Figura 2.4) e desta forma, simula padrões de maneira mais eficaz, reduzindo o tempo de CPU (TAHMASEBI et al., 2012).

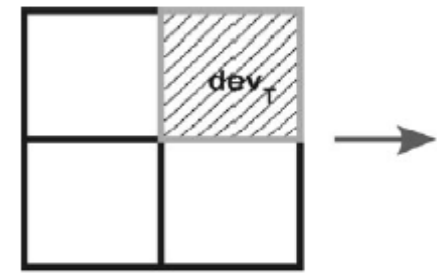

(a)

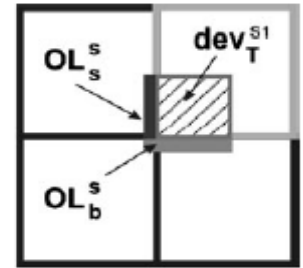

(b)

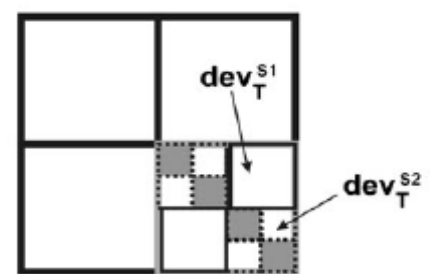

(c)

Figura 2.4 Regiões de sobreposição são mostradas pela cor cinza sombreada (Fonte: TAHMASEBI et al. 2012). 
Existem outros algoritmos de MPS descritos na literatura, dentre os quais se destacam: Simulação por amostragem direta (Direct sampling simulation -DS) (MARIETHOZ et al., 2010), simulação baseada em cumulantes (High Order Simulation-HOSIM) (MUSTAPHA E DIMITRAKOPOULOS, 2010), Impala (STRAUBHAAR et al., 2011 e STRAUBHAAR et al., 2013), simulação baseada em wavelets (Wavelets Simulation-WAVESIM) (CHATTERJEE et al., 2012), simulação por decomposição da função de distribuição acumulada (decomposition of cumulative distribution function simulation - CDFSIM) (MUSTAPHA et al., 2014), simulação híbrida baseada em pixels e padrões (Hybrid Pixel and Pattern-based Simulation-HYPPS) (TAHMASEBI, 2017).

\subsection{Imagem de Treinamento}

De acordo com Journel \& Zhang (2006), o controle de padrões estruturais ou imagens de treinamento (TI), são a representação de um modelo conceitual do fenômeno geológico, que gera a estrutura espacial da variável sejam variáveis contínuas ou categóricas que é considerado como um conhecimento a priori da continuidade espacial (ARPAT, 2005). Segundo Journel (2005), a imagem de treinamento substitui o variograma, superando as limitações para descrever a continuidade geológica, especialmente no caso de estruturas curvilíneas ou geometrias complexas bastante comuns na natureza (GUARDIANO \& SRIVASTAVA, 1993). ATI, é um requisito importante para a aplicação de técnicas de múltiplos pontos (MPS), tendo em conta que permite reproduzir de maneira mais realista os padrões geológicos contidos na TI (GUARDIANO \& SRIVASTAVA, 1993). Cabe ressaltar, que a TI deve conseguir caracterizar o comportamento local e global das estruturas.

A TI é usada somente para representar heterogeneidades geológicas, tal como a sinuosidade de um canal em um reservatório de petróleo (STREBELLE, 2000), ou relações espaciais complexas de múltiplas características na sub superfície. Além disso, a imagem de treinamento não é condicionada a pontos amostrais e não contém informações especificas do 
fenômeno em estudo, tais como localização (STREBELLE 2002; APART, 2005).

De acordo com Arpat (2005), as imagens de treinamento podem resultar da caracterização de dados de afloramentos, de um esboço geológico devidamente digitalizado (em duas ou três dimensões), bem como de realizações não condicionais da simulação baseada em objetos, ou simplesmente da interpretação digitalizada, realizada por um geólogo. Além disso, segundo Tahmasebi (2018), a TI também pode ser construída por métodos estatísticos tradicionais.

Com relação à construção das $T I$ para a simulação MPS vários métodos têm sido propostos, tais como: métodos baseados em objetos ou booleanos, uma opção para a construção de modelos categóricos (BOUCHER et al., 2010; PYRCZ et al., 2008). Essa técnica é caracterizada pela parametrização geométrica dos objetos (tamanho, direção e sinuosidade) e é uma das fontes mais aceitas e difundidas para a criação de uma $\boldsymbol{T I}$. Outra das tecnicas destacadas em Tahmasebi (2018) é conhecida como afloramento análogo que fornece imagens de geometria e continuidade espacial em $2 D$ ou $3 D$. Além disso, este modelo proporciona uma imagem única das características geológicas. Os recentes avanços nos métodos de mapeamento de afloramentos são digitais, incluindo fotogrametria, que permite a rápida aquisição de modelos $3 D$ de afloramentos virtuais de alta resolução (WANG et al., 2018). Finalmente, a terceira forma de construir TI apresentada em Tahmasebi (2018), são métodos baseados em processos, que apresentam uma maneira de representar uma heterogeneidade geológica realista em modelos de subsolo, que pretendem desenvolver modelos $3 D$ de um meio poroso. No entanto, esta técnica tem um alto custo computacional e requer cálculos consideráveis. Alguns exemplos em $2 D$ e $3 D$ de imagens de treinamento são ilustradas na Figura 2.5 . 

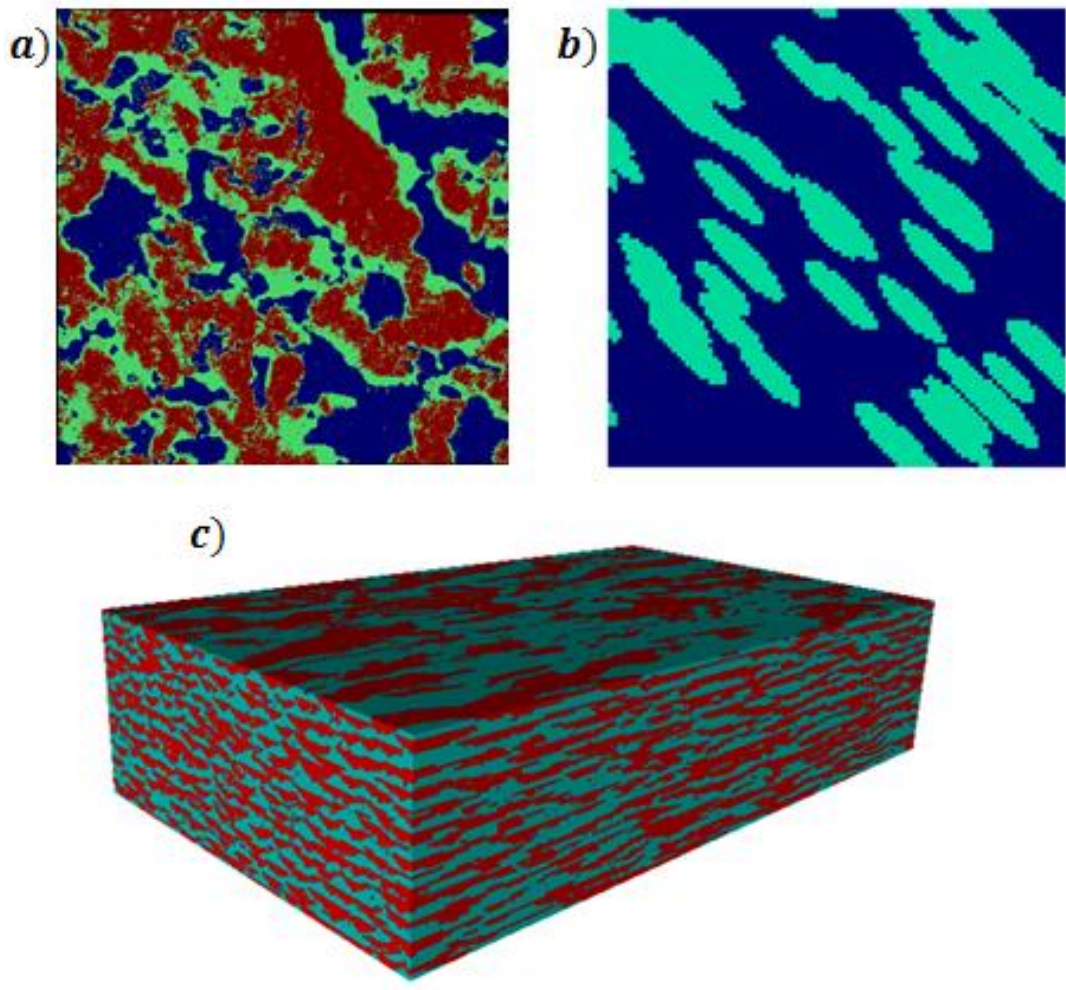

Figura 2.5 Exemplos de imagens de treinamento para simulações bidimensional e tridimensional. a) dados exaustivos de Walker Lake categorizados; b) Modelagem não estacionária; c) Malha 3D que representa as hidrofácies em um aqüífero aluvial no vale de Maules Creek, Austrália. (Fonte: MARIETHOZ E CAERS, 2014).

\subsection{Template}

A simulação estocástica baseada em padrões é fundamentada na geração de uma base de padrões a partir da varredura da imagem de treinamento, onde o template é a principal ferramenta. Definido como um conjunto de nós ou pontos usados para percorrer e encontrar os padrões semelhantes da TI. Cabe ressaltar, que o template deve estar inteiramente contido na imagem de treinamento.

Seja $\boldsymbol{G}_{\boldsymbol{t} \boldsymbol{i}}$ uma malha regular cartesiana, que subdivide a imagem de treinamento e defina $\boldsymbol{t} \boldsymbol{i}(\boldsymbol{u})$ como um valor da imagem de treinamento TI no qual $\boldsymbol{u} \in \boldsymbol{G}_{\boldsymbol{t} \boldsymbol{i}}$ (ARPAT, 2005). O conjunto de valores $\boldsymbol{t} \boldsymbol{i}(\boldsymbol{u})$ da imagem de treinamento compõe um vetor particular de múltiplos pontos dentro de um template $\boldsymbol{T}$ predefinido, centrado na coordenada $\boldsymbol{u}$, denotado por $\boldsymbol{t} \boldsymbol{t}_{T}(\boldsymbol{u})$, é: 


$$
t i_{T}(u)=\left\{t i\left(u+h_{1}\right), t i\left(u+h_{2}\right), \ldots, t i\left(u+h_{\alpha}\right), \ldots, t i\left(u+h_{n T}\right)\right\}
$$

onde os vetores $\boldsymbol{h}_{\alpha}$ definem a geometria dos $\boldsymbol{n}_{\boldsymbol{T}}$ nós do template $\boldsymbol{T}$ com $\alpha=1, \ldots, n_{T}$ e o vetor $h_{1}=\mathbf{0}$, que é equivalente à localização central do template, e $\boldsymbol{n}_{\boldsymbol{T}}$ representa o tamanho do template que é o parâmetro mais importante na modelagem baseada em padrões. Na Figura 2.6, ilustra-se a caracterização do template bidimensional com dimensões $\mathbf{5} \times \mathbf{5}$, ou seja, $\boldsymbol{n}_{\boldsymbol{T}}=25$. Em geral, a $\boldsymbol{t} \boldsymbol{i}(\boldsymbol{u})$ pode ser construída por variáveis aleatórias contínuas, discretas ou categóricas.
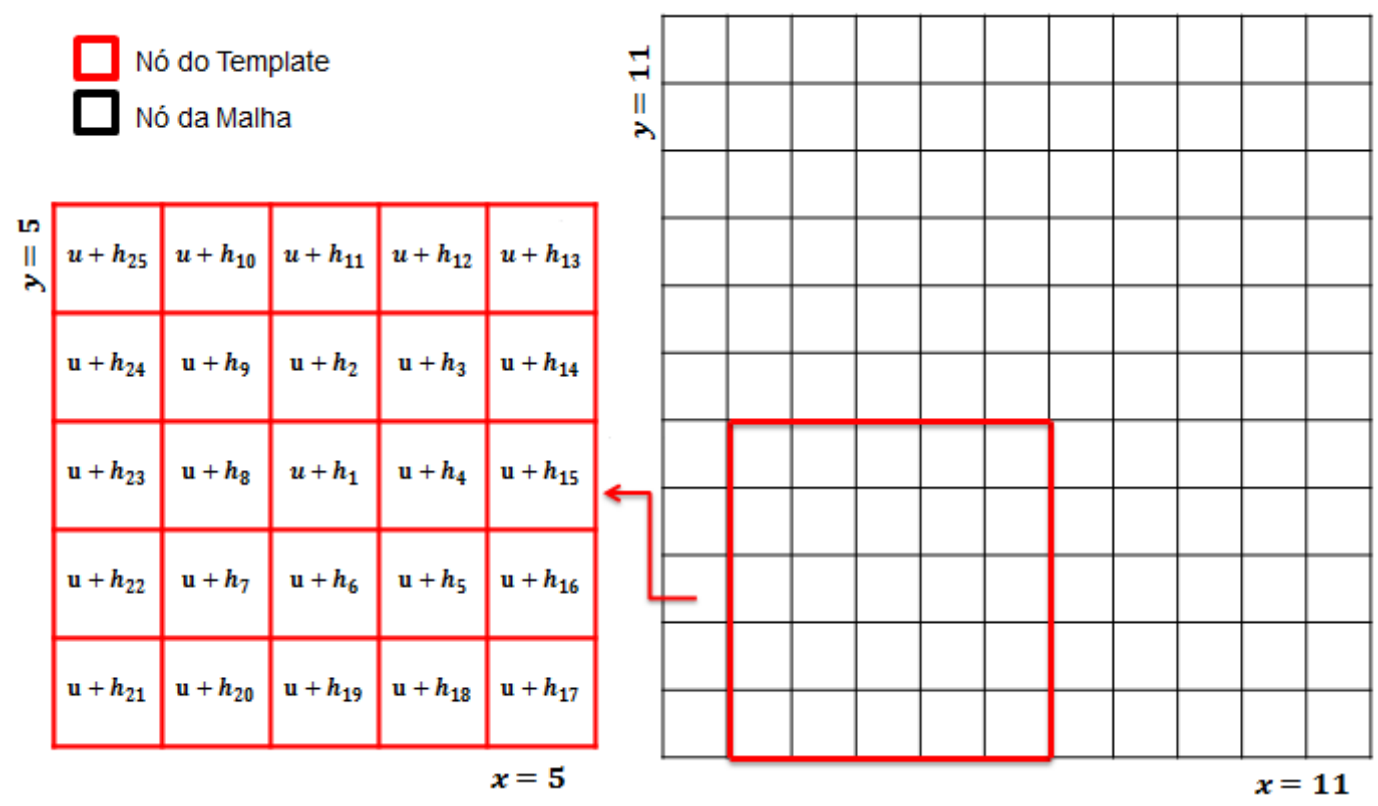

Figura 2.6 Representação de um Template bidimensional $\mathbf{5} \times \mathbf{5}$. O vetor $\mathbf{h}_{\mathbf{1}}=\mathbf{0}$ identifica a localização central $\boldsymbol{u}$ do $\boldsymbol{T}$ em uma malha com dimensões $\mathbf{1 1} \times \mathbf{1 1}$ (Fonte: ARPAT, 2005).

\subsection{Inner patch}

Devido à possibilidade de uma grande quantidade de pontos para simular pode haver uma grande carga computacional e uma alta demanda de memória durante o processo. Buscando contornar esse problema o conceito de template interno $\boldsymbol{i} \boldsymbol{p}_{\boldsymbol{T}}(\boldsymbol{u})$ (também conhecido como inner patch) foi desenvolvido. Assim como o template, o $\boldsymbol{i} \boldsymbol{p}_{\boldsymbol{T}}(\boldsymbol{u})$ é centrado em $\boldsymbol{u}$ e sua 
utilização determina que os valores centrais do padrão escolhido sejam "congelados" e removidos do caminho aleatório dentro da malha a ser simulada e usados como informações secundárias até que o processo de simulação seja concluído, ou seja, eles não serão visitados pelo algoritmo. Consequentemente, menos cálculos de similaridade são efetuados, além do que melhora o resultado da conectividade entre as características da área (MOHAMMAD et al., 2012). O $\boldsymbol{i} \boldsymbol{p}_{\boldsymbol{T}}(\boldsymbol{u})$, deve ser de tamanho menor ou igual ao template. Mostra-se na Figura 2.7 um exemplo com um template de dimensões $\mathbf{5} \times \mathbf{5}$ e com um $\boldsymbol{i} \boldsymbol{p}_{T}(\boldsymbol{u})$ bidimensional com parâmetros iguais a $\mathbf{3} \times \mathbf{3}$.

Template

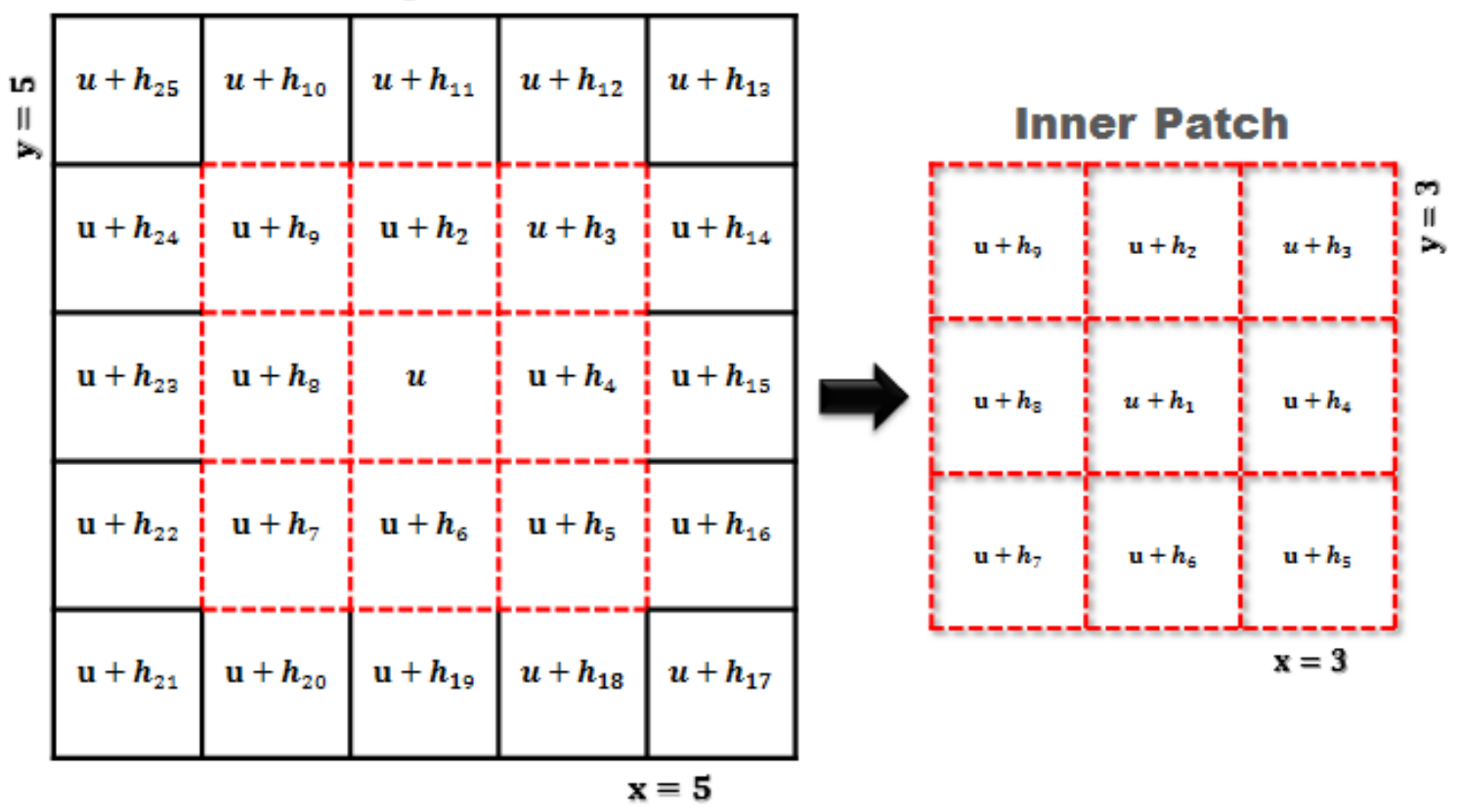

Figura 2.7 llustração do $\boldsymbol{T}$ com dimensões $5 \times 5$ e $\boldsymbol{i} \boldsymbol{p}_{T}(\boldsymbol{u}) \mathbf{3} \times \mathbf{3}$.

\subsection{Banco de Dados de Padrões}

O pré-processamento da imagem de treinamento $\boldsymbol{t} \boldsymbol{i}$, é feita uma única vez de maneira sequencial por meio de uma varredura efetuada pelo $\boldsymbol{T}$, seguida do armazenamento dos vetores de múltiplos pontos $\boldsymbol{t i}_{\boldsymbol{T}}(\boldsymbol{u})$ correspondentes numa base de dados, onde cada vetor $\boldsymbol{t} \boldsymbol{i}_{\boldsymbol{T}}(\boldsymbol{u})$ é chamado de padrão da imagem de treinamento e a base de dados formada por estes 
vetores é denotada por patdb $\mathbf{b}_{\mathbf{T}}$ (ARPAT, 2005). Na Figura 2.8, ilustra-se este processo para um template $2 D$ de tamanho $3 \times 3$.

Uma vez que a base de dados de padrões é construída patdb $\mathbf{b}_{\mathrm{T}}$, assumese independência entre os diferentes padrões na base de dados, relativo à sua localização, pois $\boldsymbol{u} \in \boldsymbol{G}_{\boldsymbol{t} i}$ não é armazenada (ARPAT, 2005). Cada padrão individual, dentro da base de dados, definido pelo mesmo template $\boldsymbol{T}$ é representado pelo vetor padrão (equação 2.2):

$$
\boldsymbol{p a t}_{T}^{k}=\left\{\operatorname{pat}_{\boldsymbol{T}}^{\boldsymbol{k}}\left(\boldsymbol{h}_{1}\right), \operatorname{pat}_{T}^{\boldsymbol{k}}\left(\boldsymbol{h}_{2}\right), \ldots, \text { pat }_{T}^{k}\left(\boldsymbol{h}_{\alpha}\right), \ldots, \operatorname{pat}_{T}^{k}\left(\boldsymbol{h}_{n T}\right)\right\}
$$

onde o índice $\boldsymbol{k}$ refere-se a um padrão específico na base de dados, com $\boldsymbol{k}=1, \ldots, \boldsymbol{n}_{\text {pat }_{T}}$ e $\boldsymbol{n}_{\text {pat }}$ são todos os padrões que compõem a base de dados. Além disso, duas ou mais configurações de padrões dentro da base dados poderiam ter a mesma singularidade em valores $\left(\boldsymbol{p a t} \boldsymbol{k}_{T}^{\boldsymbol{k}_{1}} \equiv \boldsymbol{p a t} \boldsymbol{t}_{T}^{\boldsymbol{k}_{2}}, \operatorname{com}_{\boldsymbol{1}_{1}} \neq \boldsymbol{k}_{\mathbf{2}}\right)$ (ARPAT, 2005).

As descrições das funções, para obter o banco de dados de padrões da TI e verificar que as coordenadas do template estão completamente dentro da imagem, são mostradas no quadro 2.1 .
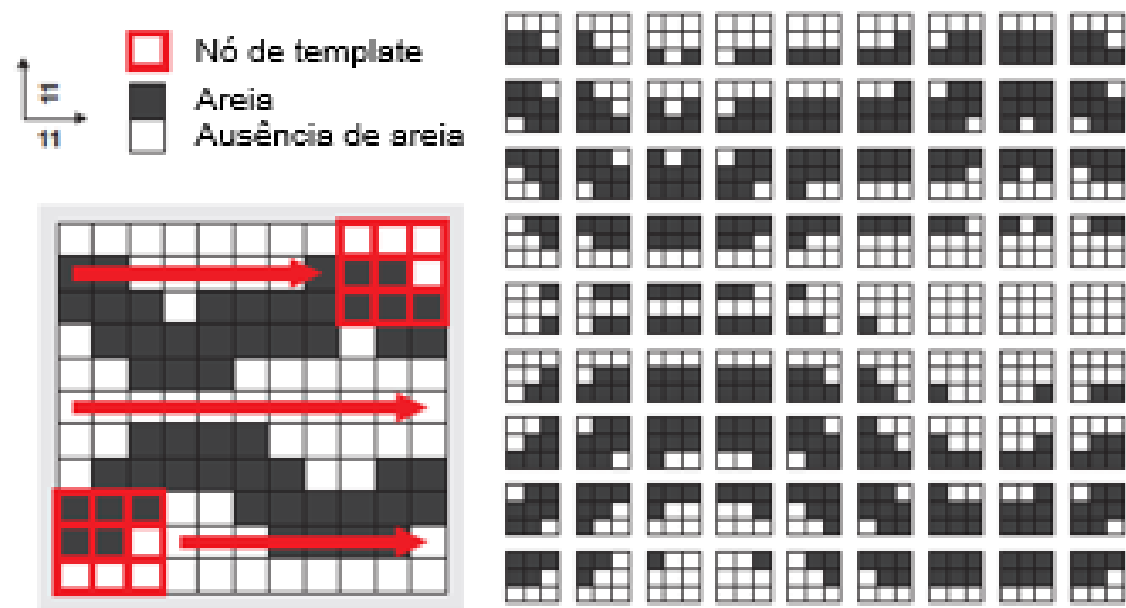

Imagem de treinamento

Banco de dados de padrões

Figura 2.8 Varredura da imagem de treinamento para a construção do banco de dados de padrões através do $\mathbf{T}$ bidimensional de tamanho 3x3. (Fonte: ARPAT, 2005). 
Quadro 2.1 Descrição das funções PatterBase e ScanTemplate.

\section{Função PatternBase e ScanTemplate}

1. A função PatternBase faz um escaneamento sequencial na $T I$ usando um $\boldsymbol{T}$, sobre a grade $\boldsymbol{G}_{t i}$, no qual se obtém todos os padrões $\boldsymbol{p a t} \boldsymbol{t}_{T}^{k}$ $\boldsymbol{k}=\mathbf{1}, \ldots, \boldsymbol{n}_{\text {pat }_{T}}$ da $\boldsymbol{T I}$.

2. A função scanTemplate verifica que a configuração do template deve estar completamente dentro da $\boldsymbol{T I}$.

\subsection{Simulação Sequencial com Padrões}

Uma vez construído, o banco de dados de padrões $\boldsymbol{p a t d}_{T}$, obtido a partir da TI, o algoritmo continua com a simulação de padrões na malha da realização $\boldsymbol{r e}$. Inicialmente, os nós dentro da malha não são informados $\boldsymbol{r e}(\boldsymbol{u}) \in \boldsymbol{G}_{\boldsymbol{r} \boldsymbol{e}}$ e, do mesmo modo, para cada realização. Nesse sentido, definimos como nós ou valores não informados ou desconhecidos como $\boldsymbol{r e}(\boldsymbol{u})=\boldsymbol{\gamma}$ que neste estudo é $\boldsymbol{r} \boldsymbol{e}(\boldsymbol{u})$ é definido como a equação (2.3):

$$
r e(u)= \begin{cases}\gamma, & \text { não informado } \\ \varphi, & \text { nó informado, nó simulado } \\ \text { ou dados de condicionamento }\end{cases}
$$

Em geral, $\boldsymbol{r} \boldsymbol{e}$ poderia ser uma variável contínua, discreta ou categórica. Ou seja, os valores vetoriais da realização são do mesmo tipo que a imagem de treinamento. Assim como os algoritmos de simulação geoestatística, a realização $\boldsymbol{r} \boldsymbol{e}(\boldsymbol{u})$ simulada com padrões usa o paradigma da simulação sequencial sendo denominada de simulação sequencial com padrões (SSP).

Antes de discorrer sobre a SSP é necessário introduzir dois conceitos importantes: 1. Evento de dados e 2. Similaridade de padrões. 


\subsection{Evento de Dados}

Durante a simulação, o $\boldsymbol{T}$ centrado em $\boldsymbol{u}$ captura um conjunto de dados que pode ser composto de nós de amostra, nós previamente simulados e nós informados $i \boldsymbol{p}_{T}(\boldsymbol{u})$, que é designado de evento de dados e é indicado por $\boldsymbol{d e v}_{T}(\boldsymbol{u})$. Vale ressaltar que o tamanho do $T$, é o mesmo que foi usado durante a escolha dos padrões de TI. Além disso, da mesma maneira que ti(u), $\boldsymbol{d e v}_{T}(\boldsymbol{u})$ é um vetor de múltiplos pontos (ARPAT \& CAERS, 2007):

$$
\boldsymbol{d e v}_{T}(\boldsymbol{u})=\left\{\operatorname{dev}_{T}\left(\boldsymbol{u}+\boldsymbol{h}_{\mathbf{1}}\right), \ldots, \operatorname{dev}_{T}\left(\boldsymbol{u}+\boldsymbol{h}_{\alpha}\right), \ldots, \operatorname{dev}_{T}\left(\boldsymbol{u}+\boldsymbol{h}_{\boldsymbol{n} T}\right)\right\}
$$

onde $\operatorname{dev}_{T}\left(\boldsymbol{u}+\boldsymbol{h}_{\alpha}\right)=\boldsymbol{r} \boldsymbol{e}\left(\boldsymbol{u}+\boldsymbol{h}_{\alpha}\right)$ e re é a realização previamente definida.

A diferença entre um evento de dados e um padrão $\boldsymbol{p a t} t_{T}^{k}$ é que o evento de dados aceita valores desconhecidos. Além disso, diferentes estados podem ser atribuídos a um nó em particular, isto é:

Nó Informado: um nó na realização $r e$ que foi estabelecido pela visita prévia a outro local $\boldsymbol{u}$.

Nó Congelado: um nó em realização $r \boldsymbol{e}$ que foi congelado por visita anterior a outro local $\boldsymbol{u}$ e foi removido do caminhamento aleatório.

Dados de Condicionamento: um nó na realização de re que foi inicialmente estabelecido como dados duros para o condicionamento.

Os diferentes estados de $\operatorname{dev}_{T}\left(\boldsymbol{u}\right.$ ) podem ser penalizados por $\boldsymbol{\omega}_{i}$, (no momento do cálculo de similaridade), ou seja, cada tipo de nó é multiplicado por um peso ou valor numérico. Por exemplo, $\boldsymbol{\omega}_{\mathbf{1}}=\mathbf{0 . 5}$ dados de condicionamento, $\boldsymbol{\omega}_{2}=\mathbf{0 . 3}$ simulado ou previamente simulado, $\boldsymbol{\omega}_{3}=\mathbf{0 . 2}$ nós informados. Os diferentes valores ou pesos $\omega_{i}$ são definidos conforme a equação (2.5):

$$
\omega_{i}=\left\{\begin{array}{l}
\omega_{1}, \text { dados de condicionamento } \\
\omega_{2}, \text { simulados } \\
\omega_{3}, \text { nós informados }
\end{array}\right.
$$


onde $\omega_{i}(i=1,2,3)$ é o peso relacionado a cada tipo de evento de dados. Além disso, está sujeito $\sum_{i=1}^{3} \omega_{i}=1$ e $\omega_{i} \geq 0$ Para isso, o algoritmo contém uma função AssignWeight, onde os pesos $\omega_{i}$ são os parâmetros de entrada.

\subsection{Similaridade de Padrões}

O conceito de medida de similaridade e distância é cada vez mais usado nas ciências aplicadas, para calcular as similaridades/distâncias entre dois objetos, sendo essencialmente utilizadas no reconhecimento de padrões que incluem, por exemplo, a classificação (BANDYOPADHYAY \& SAHA, 1998).

A similaridade entre objetos é associada a um valor numérico ou escalar, calculado por meio da função genérica de similaridade $s\langle\boldsymbol{x}, \boldsymbol{y}\rangle$ entre os vetores $\boldsymbol{x}$ e $\boldsymbol{y}$ e, no contexto da simulação, são eventos de dados e/ou padrões. O resultado da função genérica determina quão similar são os vetores $\boldsymbol{x}$ e $\boldsymbol{y}$. Por convenção, na simulação baseada em padrões, o evento de dados é sempre escrito primeiramente em referência ao padrão dentro da base de dados, isto é, $\boldsymbol{s}\left\langle\boldsymbol{d e v}_{T}(\boldsymbol{u}), \boldsymbol{p a t} \boldsymbol{t}_{T}^{k}\right\rangle$ que significa "o quão similar ao padrão é o evento de dado" (ARPAT, 2005).

Define-se matematicamente à distância sobre um conjunto $x$ como uma função não negativa $\boldsymbol{d}$ para o par de elementos ou vetores em $\boldsymbol{x}$ sobre os reais positivos, com as seguintes propriedades:

1) Positiva: $\mathbf{d}\langle\mathbf{x}, \mathbf{y}\rangle \geq \mathbf{0}$

2) Idêntico: $d\langle x, y\rangle=0$, se e somente se, $x=y$

3) Simetria: $d\langle x, y\rangle=d\langle y, x\rangle$

4) Desigualdade triangular: $\mathbf{d}\langle\mathbf{x}, \mathbf{z}\rangle \leq \mathbf{d}\langle\mathbf{x}, \mathbf{y}\rangle+\mathbf{d}\langle\mathbf{y}, \mathbf{z}\rangle$

Segundo Arpat (2005) unicamente as duas primeiras propriedades são utilizadas na prática. Quanto à semelhança entre objetos existe na literatura uma grande quantidade de medidas que quantificam está relação entre 
diferentes características (BANDYOPADHYAY \& SAHA, 1998), mas neste estudo quatro distâncias serão testadas: a distância de Manhattan, a distância Euclidiana, a distância de Lorentz e a distância Cosseno.

\subsubsection{Distância de Manhattan}

A métrica de Minkowski, introduzida no século XIX, é uma das funções de distância mais usadas devido à sua simplicidade. Atribuída ao caso de $\boldsymbol{d}\left\langle\boldsymbol{\boldsymbol { d e v } _ { T }}(\boldsymbol{u}), \boldsymbol{p a t} \boldsymbol{T}_{T}^{k}\right\rangle$ para um evento de dados e um padrão (ARPAT, 2004), é obtida a equação 2.6 .

$$
d\left\langle\operatorname{dev}_{T}(u), \operatorname{pat}_{T}^{k}\right\rangle=\left(\sum_{\alpha=0}^{n_{T}-1}\left|\operatorname{dev}_{T}\left(u+h_{\alpha}\right)-\operatorname{pat}_{T}^{k}\left(h_{\alpha}\right)\right|^{p}\right)^{1 / p}
$$

Sendo que para $\boldsymbol{p}=\mathbf{1}$ ou $\boldsymbol{p}=\mathbf{2}$, a métrica de Minkowski torna-se igual à métrica de Manhattan e Euclidiana, respectivamente. À distância Manhattan é uma medida de similaridade simples, mas útil, que permite quantificar o grau de similaridade entre o evento de dados e os padrões (ARPAT \& CAERS, 2007), conforme apresentado na equação 2.7.

$$
d\left\langle\operatorname{dev}_{T}(u), \operatorname{pat}_{T}^{k}\right\rangle=\sum_{\alpha=0}^{n_{T}-1} \omega_{i} \cdot\left|\operatorname{dev}_{T}\left(u+h_{\alpha}\right)-\operatorname{pat}_{T}^{k}\left(h_{\alpha}\right)\right|
$$

Onde:

- $\boldsymbol{n}_{T}$ : número total de nós no template;

- $\omega_{i}$ : peso assoc iado a cada nó de $\boldsymbol{T}$;

- $\boldsymbol{u}$ : nó central do evento de dados;

- $\boldsymbol{h}_{\boldsymbol{\alpha}}$ : deslocamento do nó no template.

Nenhuma das distâncias permite valores desconhecidos ou ausentes, pois exige que os vetores sejam totalmente definidos. No entanto, um evento de dados $\boldsymbol{d e v}_{T}(\boldsymbol{u})$ pode ter nós desconhecidos, ou seja, $\boldsymbol{d e v}_{\boldsymbol{T}}\left(\boldsymbol{u}+\boldsymbol{h}_{\boldsymbol{\alpha}}\right)=\boldsymbol{\gamma}$, para alguns $\boldsymbol{h}_{\boldsymbol{\alpha}}$. Embora na prática os valores desconhecidos não sejam 
considerados no cálculo da distância, ou seja, omitidos durante seu cálculo. $\mathrm{Na}$ Figura 2.9, exemplifica-se o cálculo da distância Manhattan. A distância de Manhattan dos padrões de dados, na Figura 2.9a, é igual a 5 e a similaridade de $\boldsymbol{d e v}_{T}(\boldsymbol{u})$ e $\boldsymbol{p a t} \boldsymbol{t}_{T}^{k}$, na Figura $2.9 b$, é igual a quatro (os padrões com a menor distância são os mais semelhantes ao evento de dados) e. Deve-se ressaltar que quanto maior a distância menor é a similaridade.

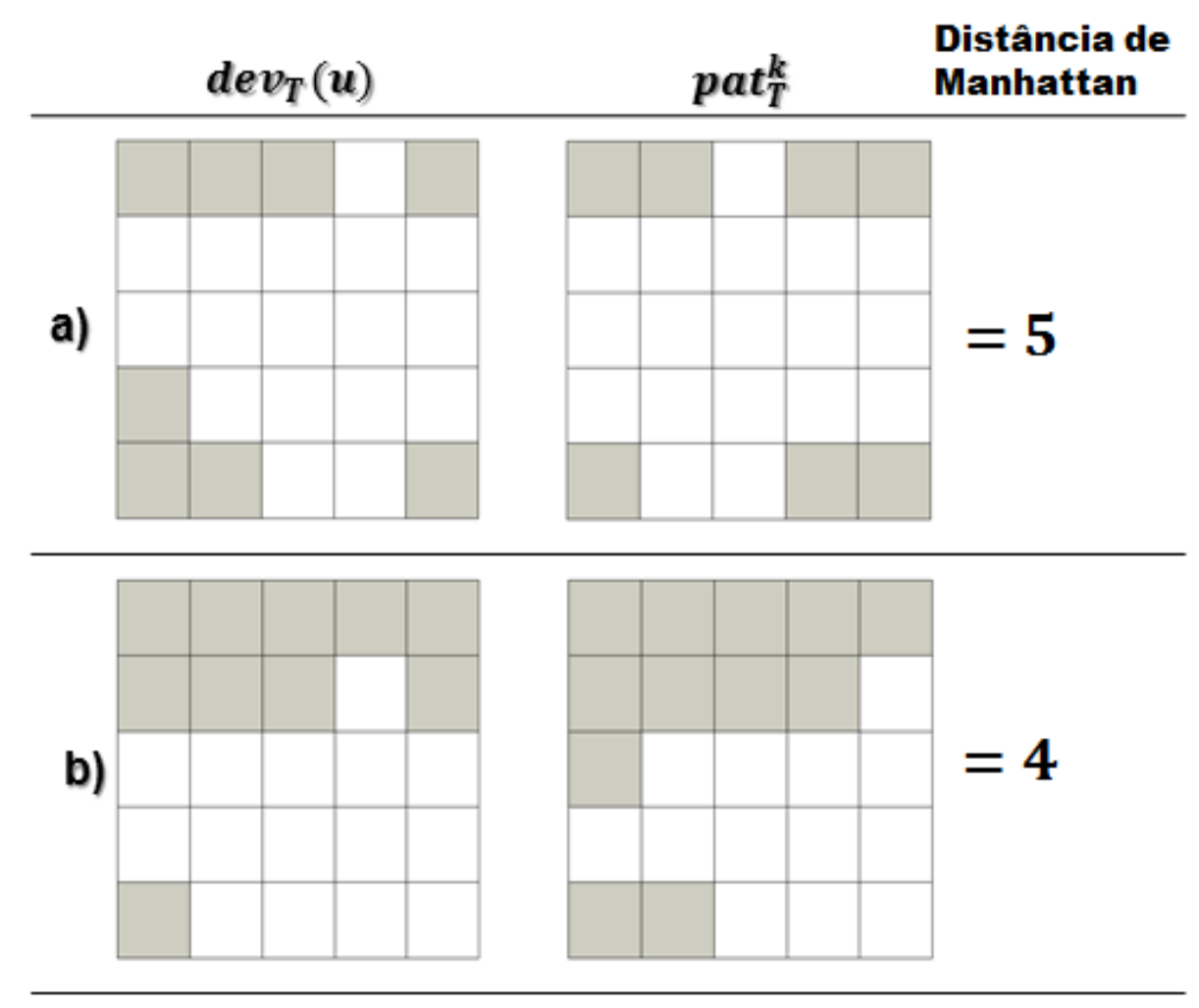

Figura 2.9 Cálculo de similaridade de padrões binários, aplicando a distância de Manhattan (Fonte: ARPAT, 2005).

\subsubsection{Distância Euclidiana}

A distância Euclidiana é a medida mais comumente utilizada para quantificar a similaridade/distância entre variáveis ou padrões. A distância Euclidiana entre um evento de dados $\boldsymbol{d e v}_{T}(\boldsymbol{u})$ e um padrão $\boldsymbol{p a t} t_{T}^{k}$ é apenas a raiz quadrada da soma das diferenças quadradas entre os dois padrões entre os pixels e é definida como (equação 2.8 ): 


$$
d\left\langle\operatorname{dev}_{T}(u), p a t_{T}^{k}\right\rangle=\sqrt{\sum_{\alpha=0}^{n_{T}-1} \omega_{\alpha} \cdot\left(\operatorname{dev}_{T}\left(u+h_{\alpha}\right)-p a t_{T}^{k}\left(h_{\alpha}\right)\right)^{2}}
$$

Onde:

- $\boldsymbol{n}_{T}$ : Número de nós do template

- $\omega_{\alpha}$ : Peso associado a cada nó do $\boldsymbol{T}$

- $\boldsymbol{d e v}_{T}\left(\boldsymbol{u}+\boldsymbol{h}_{\alpha}\right)$ e $\boldsymbol{p a t} \boldsymbol{t}_{T}^{k}\left(\boldsymbol{h}_{\alpha}\right)$ são os valores do evento de dados e padrão em $\boldsymbol{h}_{\boldsymbol{\alpha}}$, respectivamente.

No exemplo da Figura 2.10, são utilizados os mesmos padrões de dados da Figura 2.9, tomados ao caso, para aplicar a distância Euclidiana. Onde $\boldsymbol{d e v}_{\boldsymbol{T}}(\boldsymbol{u})$ e $\boldsymbol{p a t}_{T}^{k}$ são o padrão de dados mais semelhante.

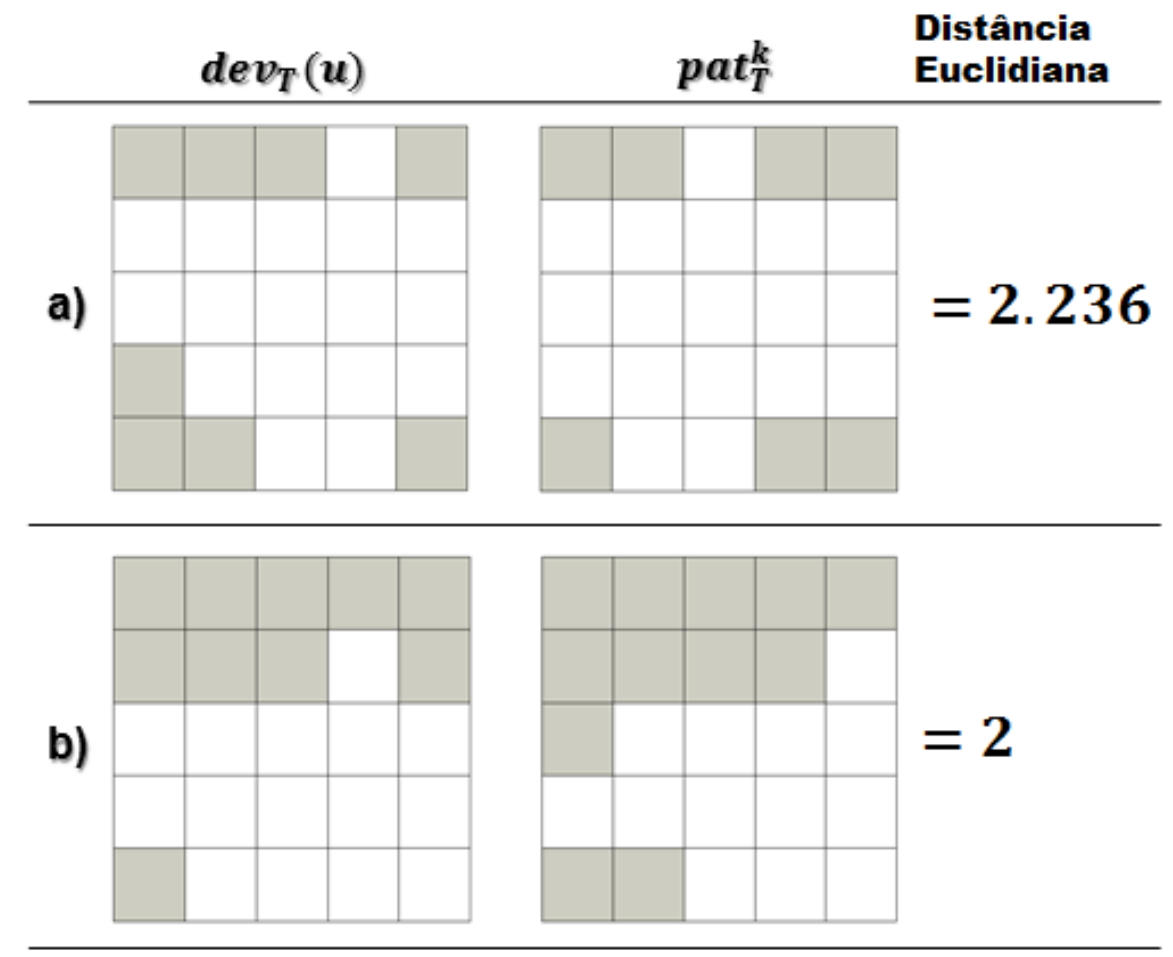

Figura 2.10 Cálculo da similaridade de padrões binários, fazendo uso da distância Euclidiana. 


\subsubsection{Distância Lorentz}

A distância de Lorentz também faz parte da família Minkowski, fundamentalmente baseada em encontrar a diferença absoluta. A métrica Lorentz é sensível a pequenas mudanças, dado que, a escala logarítmica expande a faixa inferior e comprime a faixa superior (DEZA, 2009). A distância Lorentziana entre um evento de dados e um padrão é representada pelo logaritmo natural da diferença absoluta entre dois padrões e é definida pela como apresentado na equação 2.9 :

$$
d\left\langle\operatorname{dev}_{T}(u), p a t_{T}^{k}\right\rangle=\sum_{\alpha=0}^{n_{T}-1} \ln \left(1+\omega_{i} \cdot\left|\operatorname{dev}_{T}\left(u+h_{\alpha}\right)-p a t_{T}^{k}\left(h_{\alpha}\right)\right|\right)
$$

Onde $\mathbf{l n}$ é o logaritmo natural, se adiciona um para garantir a propriedade da não negatividade e evitar o registro de zero. No exemplo da Figura 2.11, também são utilizados os mesmos padrões das Figuras 2.9 e 2.10.



Figura 2.11 Cálculo de similaridade de padrões binários, aplicando a distância de Lorentz. 


\subsubsection{Distância Cosseno}

A distância do cosseno é algumas vezes conhecida como a métrica angular. De acordo com suas similaridades sintáticas a distância do cosseno pertence à família do produto interno. Esta distância é frequentemente, usada principalmente em espaços positivos de alta dimensão, onde a saída é limitada ao intervalo de $[\mathbf{0}, \mathbf{1}]$. A distância do cosseno é definida como:

$$
\cos \theta=\frac{\operatorname{dev}_{T}(u) \cdot p a t_{T}^{k}}{\left\|\operatorname{dev}_{T}(u)\right\|\left\|p a t_{T}^{k}\right\|}=\frac{\sum_{\alpha=0}^{n_{T}} \operatorname{dev}_{T}(u) \cdot p a t_{T}^{k}\left(h_{\alpha}\right)}{\sqrt{\sum_{\alpha=0}^{n_{T}}\left(\operatorname{dev}_{T}(u)\right)^{2}} \sqrt{\sum_{\alpha=0}^{n_{T}}\left(p a t_{T}^{k}\left(h_{\alpha}\right)\right)^{2}}}
$$

quando $\cos \theta=1$ equivale ao ângulo igual a $0^{\circ}$ e seus vetores são orientados em direções idênticas, em outras palavras, $\boldsymbol{d e v}_{T}(\boldsymbol{u})$ e $\boldsymbol{p a t} t_{T}^{k}$ são completamente semelhantes entre si, exceto por suas magnitudes. Se $\cos \boldsymbol{\theta}=\mathbf{0}$ seu ângulo correspondente é de $\mathbf{9 0}^{\circ}$, pois as variáveis dadas são perpendiculares, pode-se afirmar que os pontos de dados não são similares (MURTY \& RAGHAVA, 2016). No exemplo da Figura 2.12, também são utilizados os mesmos padrões das três figuras anteriores. 


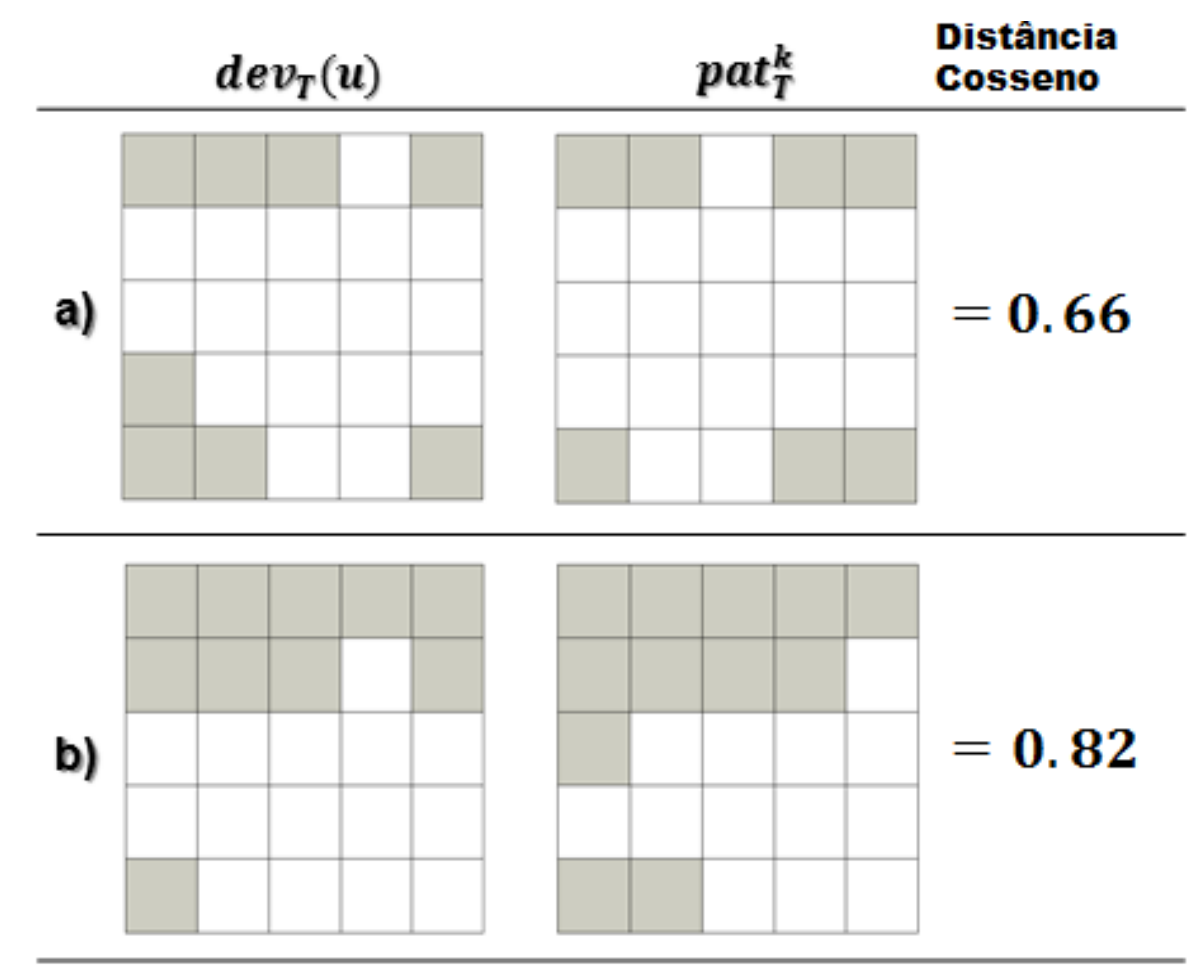

Figura 2.12 Distância Cosseno, utilizando a amostras de padrões binários.

Os processos evento de dados e similaridade são utilizados sob a função chamada SimulationPath. Essa função percorre aleatoriamente os nós da malha a simular de acordo com um caminho aleatório gerado. Além dessa função, existem outras funções internas que permitem a criação de uma realização condicional ou não condicional do fenômeno em estudo, da mesma forma, o padrão mais semelhante é calculado usando uma das medidas de distância descritas. Ambas as funções são ilustradas nas Figuras 2.14 e 2.15 e detalhada nos quadros 2.2 e 2.3 . 
Quadro 2.2 Descrição da função getDataEvent.

\section{Função getDataEvent}

1. Geração de uma malha para a realização $\boldsymbol{G}_{\boldsymbol{r e}}$, que inicialmente é completamente desinformada, embora, se houver dados condicionais ou brutos da área, então $\boldsymbol{G}_{\boldsymbol{r} e}$ conterá informações. Essas informações são atribuídas ao nó mais próximo dentro da malha, que será simulada pela função AssignData, conforme pode ser visto na Figura 2.14.

2. Um caminho aleatório é gerado com a função Ordepath, o qual o template visita cada nó $\boldsymbol{u} \in \boldsymbol{G}_{\boldsymbol{r} \boldsymbol{e}}$ da malha apenas uma vez, como mostrado na Figura 2.13.

3. A função getDataEvent mapeia dados de condicionamento (dados brutos, simulados anteriormente e outros) dentro do template $\boldsymbol{T}$ centrado no nó a ser simulado

\begin{tabular}{|c|c|c|c|c|c|c|c|c|c|c|}
\hline 120 & 71 & 42 & 52 & 88 & 93 & 28 & 30 & 72 & 95 & 36 \\
\hline 96 & 16 & 8 & 99 & 6 & 40 & 3 & 77 & 48 & 9 & 91 \\
\hline 110 & 51 & 13 & 11 & 15 & 25 & 56 & 34 & 73 & 92 & 45 \\
\hline 105 & 94 & 85 & 89 & 55 & 58 & 32 & 43 & 63 & 2 & 64 \\
\hline 50 & 90 & 59 & 119 & 44 & 103 & 108 & 46 & 114 & 76 & 79 \\
\hline 31 & 54 & 37 & 69 & 80 & 12 & 116 & 26 & 109 & 57 & 68 \\
\hline 61 & 35 & 21 & 65 & 100 & 84 & 5 & 17 & 111 & 19 & 82 \\
\hline 4 & 115 & 53 & 1 & 67 & 24 & 78 & 7 & 49 & 98 & 20 \\
\hline 14 & 118 & 70 & 101 & 74 & 86 & 10 & 18 & 22 & 102 & 83 \\
\hline 75 & 113 & 39 & 38 & 112 & 29 & 104 & 62 & 106 & 121 & 47 \\
\hline 117 & 60 & 97 & 66 & 41 & 107 & 87 & 27 & 23 & 33 & 81 \\
\hline
\end{tabular}

Figura 2.13 Caminho aleatório de todos os nós do $\mathrm{G}_{\mathrm{re}}$. 
Quadro 2.3 Descrição das funções similarPattern, disDistance e pastePattern.

\section{Função similarPattern, disDistance e pastePattern}

1. Para cada nó visitado aleatoriamente o evento de dados para cada realização é calculado, se o primeiro $\operatorname{dev}_{T}(\boldsymbol{u})$ for completamente desconhecido, então seleciona-se aleatoriamente o padrão pat $\boldsymbol{p}_{T}^{k}$ de $\boldsymbol{p a t d b}_{T}$, como o padrão pat* mais semelhante, Figura 2.15.

2. De acordo com o caminhamento aleatório já estabelecido, o próximo nó é visitado. Se existir pelo menos um $\boldsymbol{d e v}_{T}(\boldsymbol{u})$ com informação, deve-se encontrar o patrão mais semelhante $\boldsymbol{p a t}_{\boldsymbol{T}}^{*}$ ao evento que minimize $\boldsymbol{d}\left\langle\boldsymbol{d e v}_{T}(\boldsymbol{u}), \boldsymbol{p a t} \boldsymbol{T}_{T}^{k}\right\rangle$ para $\boldsymbol{k}=1, \ldots, \boldsymbol{n}_{\text {pat }_{T}}$ em $\boldsymbol{p a t d}_{\boldsymbol{T}}$ através de uma função de distância (ou seja, Manhattan, Euclidiana ou Cosseno). Esta função considera apenas os nós informados, dentro do template de pesquisa. Sendo chamado de disDistance.

3. Se houver vários $\boldsymbol{p a t} t_{T}^{k}$ semelhantes ao $\boldsymbol{d e v}_{T}(\boldsymbol{u})$ um deles é selecionado aleatoriamente como o $\boldsymbol{p a t}_{T}^{*}$ mais semelhante ao evento $\boldsymbol{d e v}_{\boldsymbol{T}}(\boldsymbol{u})$ função similarPattern.

4. Uma vez encontrado o padrão mais semelhante atribui-se $\boldsymbol{p a t}_{\boldsymbol{T}}^{*}$ a $\boldsymbol{p a t} \boldsymbol{t}_{\boldsymbol{T}}^{\boldsymbol{k}}$, ou seja $\operatorname{dev}_{T}\left(u+h_{\alpha}\right)=\operatorname{pat}_{T}^{*}\left(h_{\alpha}\right)$.

5. A função pastePattern cola o $\boldsymbol{p a t}_{T}^{k}$ escolhido anteriormente no $\boldsymbol{G}_{\boldsymbol{r e}}$. Pelo qual o nó central do template é simulado e aqueles valores internos do template que pertencem ao $\boldsymbol{i p}_{T}(\boldsymbol{u})$ são congelados durante o processo de simulação. Cabe ressaltar que, se dentro do template interno de congelamento, existem nós com dados de condicionamento ou dados simulados anteriormente, eles não são substituídos por novos valores. Finalmente, o template se move para outro nó a ser simulado.

6. O template deve visitar cada nó na malha de simulação até que ela seja totalmente percorrida. 

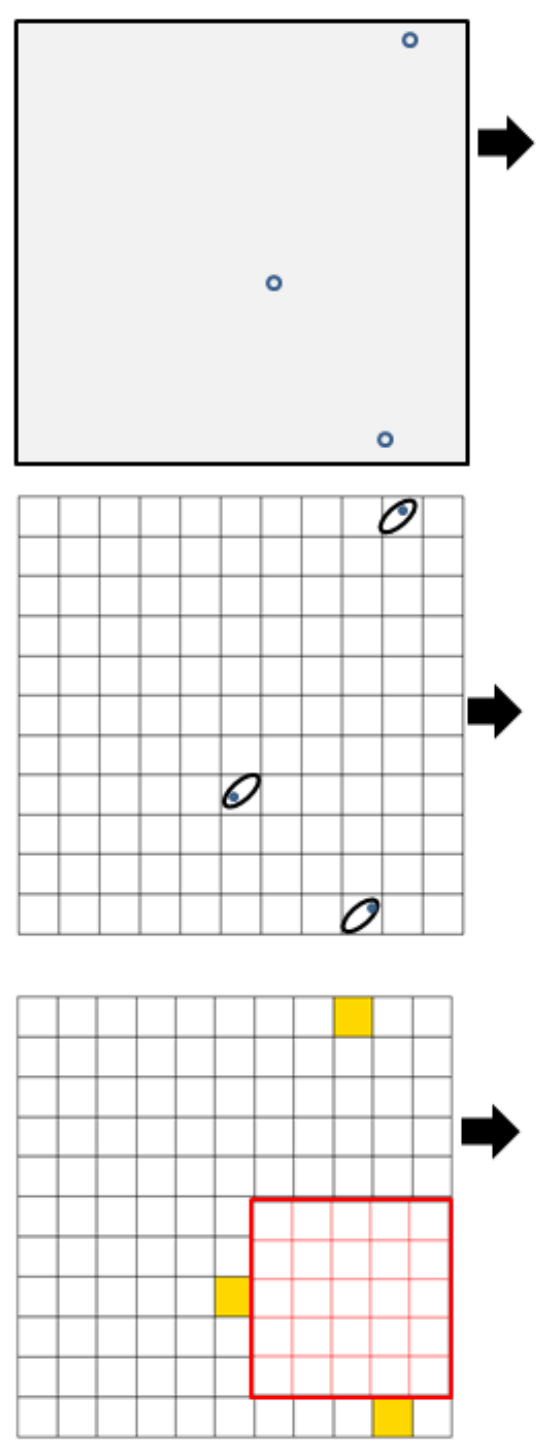

Dados amostrados em uma área de estudo.

É definida uma malha na área de interesse.

Quando existem amostras ou dados de condicionamento, a função AssignData atribui os dados da amostra original aos nós mais Próximos da grade múltipla que serem simulados
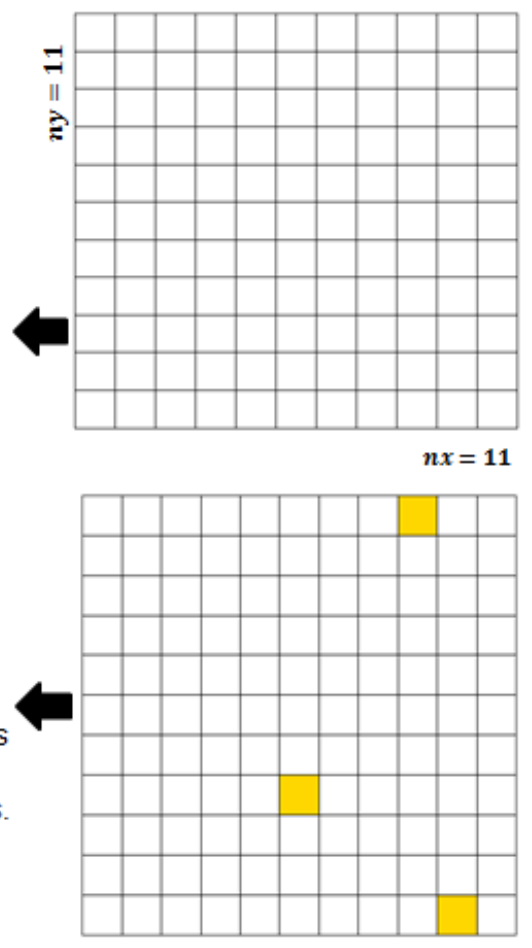

A função Ordepath gera um caminho aleatório para o template.

se o evento de dados não contem dados condicionantes, um padrão é selecionado aleatoriamente do $\boldsymbol{p a t d} \boldsymbol{b}_{T}$.

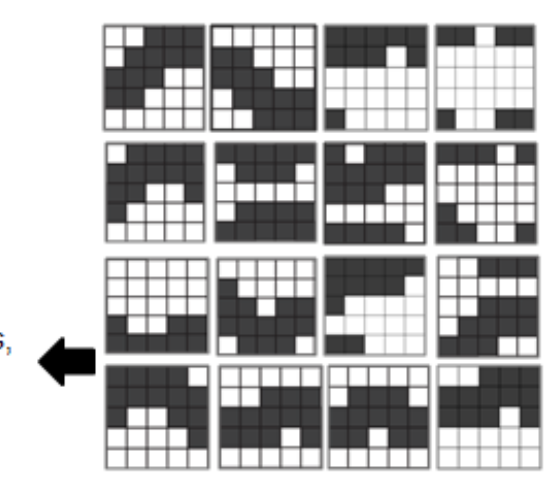

Figura 2.14 Passo a passo do algoritmo, usando a $\boldsymbol{T I}$ e o banco de dados de padrões da Figura 2.8 e um template $\mathbf{5} \times \mathbf{5}$, além disso, são utilizados dados amostrados em uma área de estudo (Fonte: modificada ARPAT, 2005). 


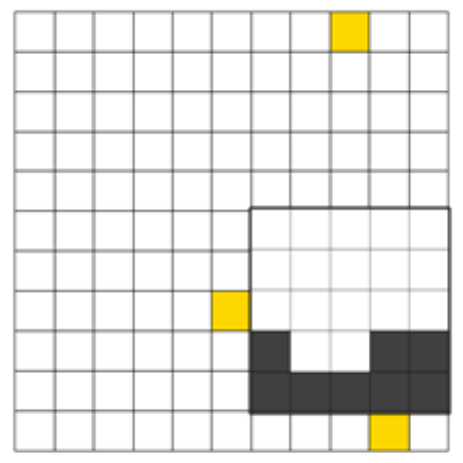

Mova o template para o próximo nó de acordo com Ordepath. Como o evento de dados também está vazio, outro pat $t_{T}^{k}$ aleatóriamente é selecionado
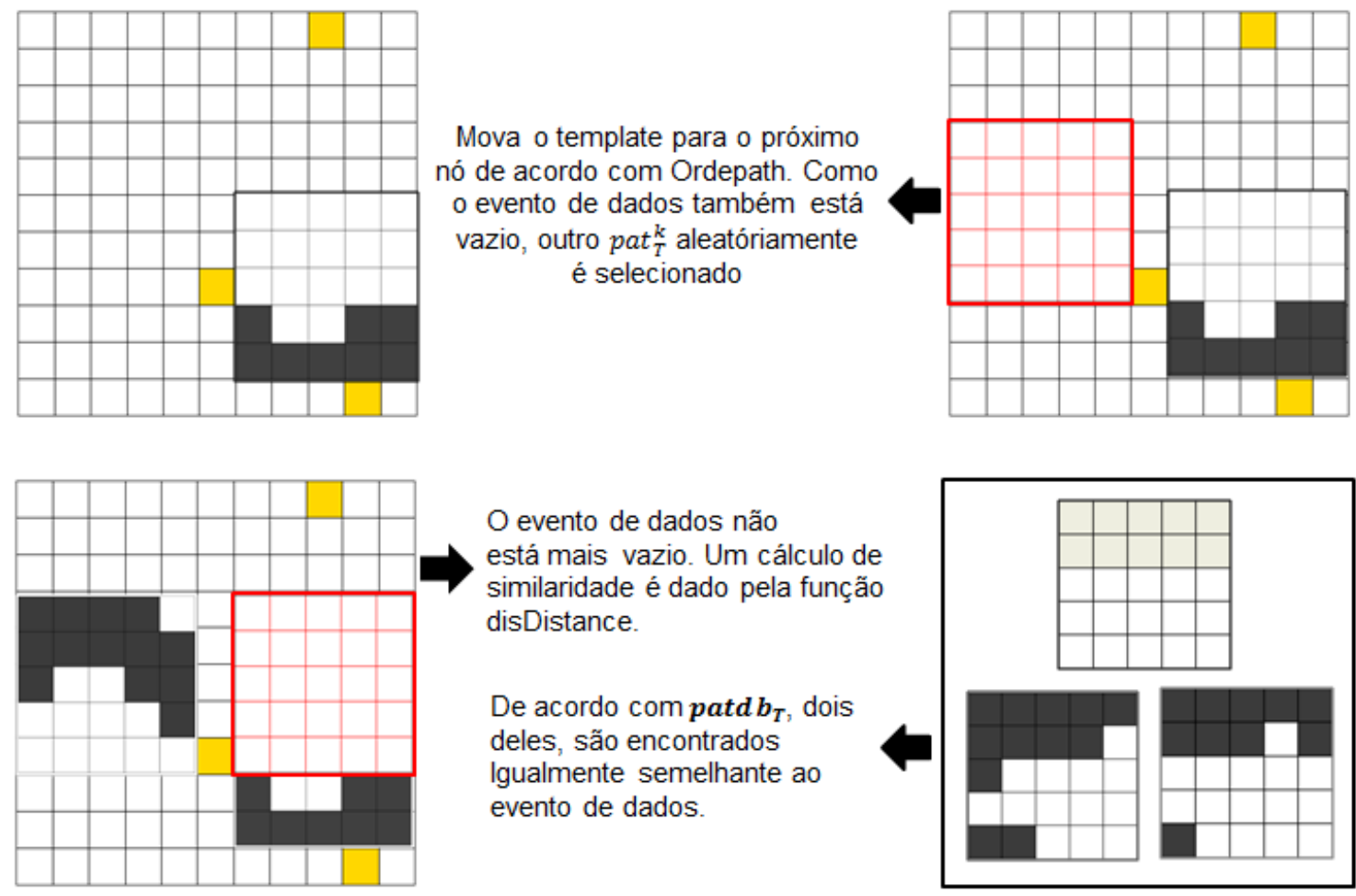

O evento de dados não está mais vazio. Um cálculo de similaridade é dado pela função disDistance.

De acordo com patd $_{T}$, dois deles, são encontrados Igualmente semelhante ao evento de dados.
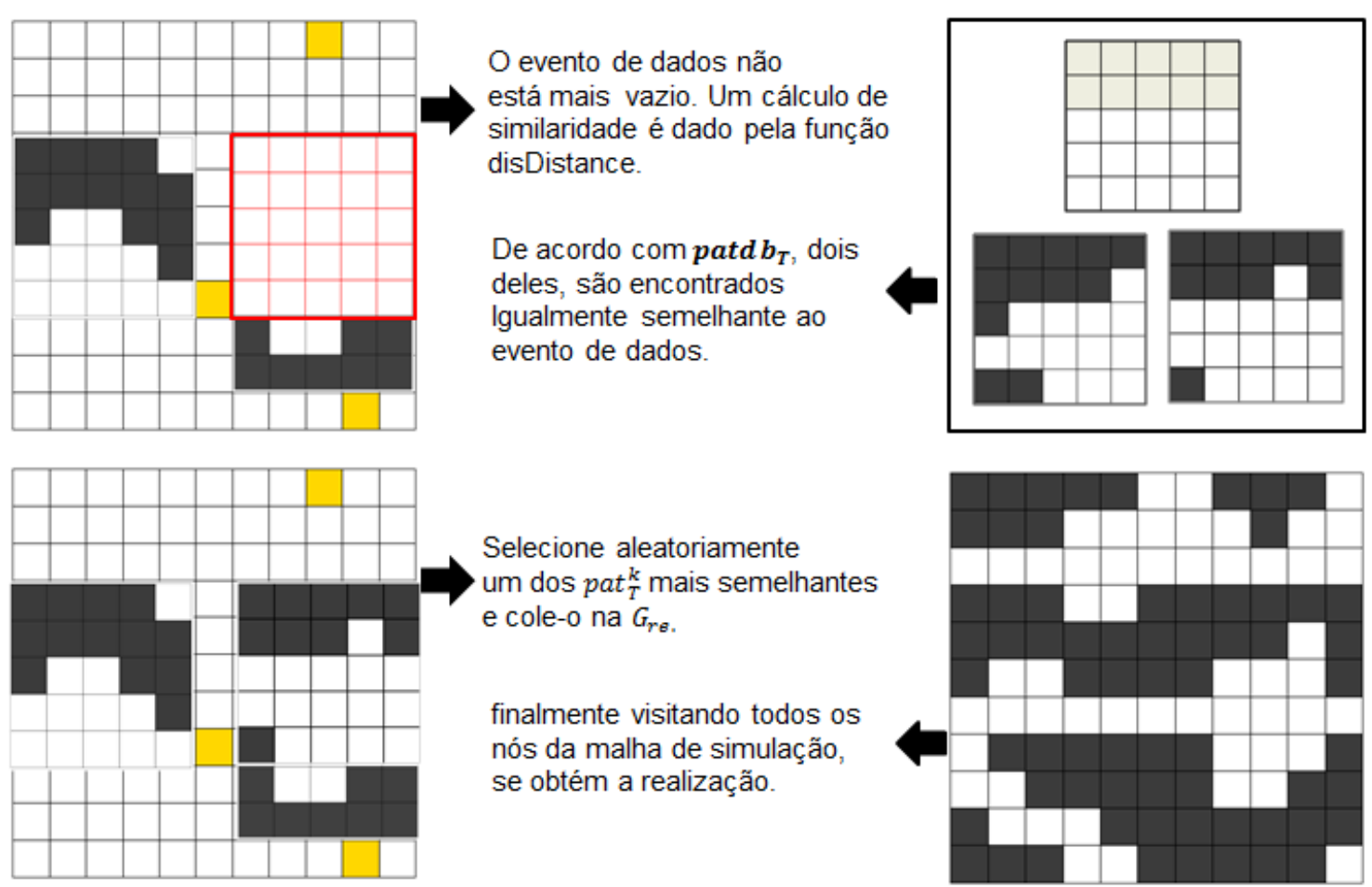

Selecione aleatoriamente um dos pat $t_{T}^{k}$ mais semelhantes e cole-o na $G_{r e}$.

finalmente visitando todos os nós da malha de simulação, se obtém a realização

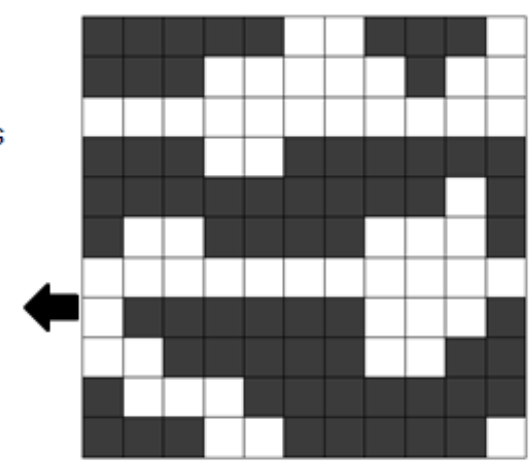

Figura 2.15 Continuação da Figura 2.14. Aplicando o algoritmo passo a passo até que uma realização seja obtida (Fonte: modificada ARPAT, 2005).

\subsection{Múltiplas Malhas (Multi Grids)}

De acordo com Honarkhah \& Caers (2010), a utilização de múltiplosgrids, ou simulação em cascata, foi proposta pela primeira vez devido ao problema originado quando se trabalha com malhas de simulação de grandes dimensões, o que implica em alta demanda de CPU e memória RAM. O conceito de múltiplos grids foi inicialmente proposto por Gómez-Hernández (1991), e posteriormente desenvolvido por Tran (1994). Caers \& Journel (1998) 
propuseram a reprodução de estatísticas de ordem superior extraídas da imagem de treinamento.

Segundo Tran (1994), a simulação com o uso de múltiplos-grids é realizada para capturar estruturas geológicas de grande escala, através da adoção de um template de grandes dimensões, porém com um número relativamente pequeno de nós. De acordo com Arpat (2007), a filosofia dos múltiplos-grids inicialmente simular malhas grossas e posteriormente simular malhas mais finas (Figura 2.16). É preciso indicar que o número de nós é igual em ambos os casos, mudando apenas a distância entre eles.

Segundo Arpat (2007), a abordagem de múltiplos grids em uma malha $\boldsymbol{G}$, é essencialmente um conjunto de simulações em cascata $\boldsymbol{G}^{\boldsymbol{g}}$ e templates dispersos $T^{g}$, ao invés de uma única grade fina e um template denso em uma malha cartesiana. Conforme o critério de coarsening, definido por Arpat (op. cit.), a aproximação é feita, considerando um número $\boldsymbol{n}_{\boldsymbol{g}}$ de grades, cada vez mais finas, onde $\boldsymbol{g}=\mathbf{1}, \ldots, \boldsymbol{n}_{\boldsymbol{g}}$ e $\boldsymbol{n}_{\boldsymbol{g}}$ é o número total de múltiplos-grids, para o grid $\boldsymbol{G}$. Assim, o $\boldsymbol{g}$ - ésimo grid $\left(\mathbf{1} \leq \boldsymbol{g} \leq \boldsymbol{n}_{\boldsymbol{g}}\right)$ é constituído a cada $\mathbf{2}^{\boldsymbol{g}-\mathbf{1} \text { - ésimo }}$ nó da malha de simulação final $(\boldsymbol{g}=\mathbf{1})$, ou seja, cada aumento no tamanho implica que as distâncias entre nós duplicam. Em consequência, os nós de $\boldsymbol{T}$ também se expandem com espaço de $\mathbf{2}^{(\boldsymbol{g}-\mathbf{1})}$, ou seja, $\boldsymbol{T}^{g}$ tem o mesmo número de nós que o template $\boldsymbol{T}$, mas possui uma maior extensão espacial, Figura 2.16. O template $\boldsymbol{T}^{g}$ usado para a grade $\boldsymbol{G}^{\boldsymbol{g}}$ é definido vetorialmente como $h_{\alpha}^{g}=2^{g-1} \cdot h_{\alpha}$, isto é: $T_{\alpha}^{g}=\left\{2^{g-1} h_{1}, \ldots, 2^{g-1} h_{\alpha}\right\}$.

Em virtude do exposto, o método consiste que o template maior, seja utilizado nas malhas mais grossas (coarse grids) com o objetivo de capturar as estruturas de grande escala localizada na imagem de treinamento e que templates de tamanho menor reproduzam informações em pequenas escalas nos grids mais finos (fine grids).

Quando é usado, o múlti-grid no algoritmo, o processo é semelhante ao que já foi mencionado, com a única diferença, de que o template utilizado é o grosso $\boldsymbol{T}^{g}$ em vez de $\boldsymbol{T}$. Segundo Arpat (2005), para a extração dos padrões

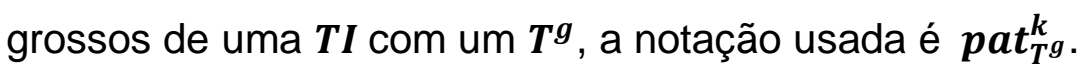




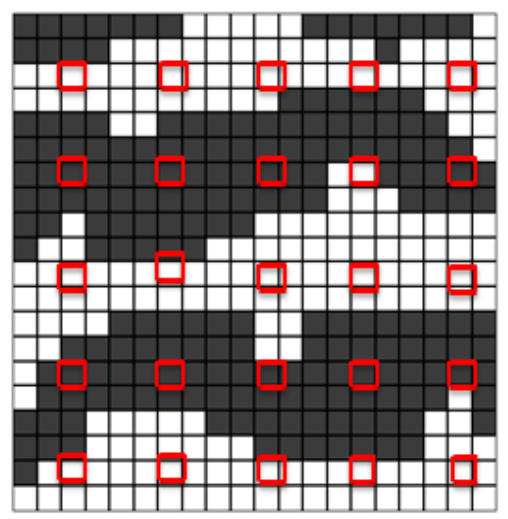

Grid grosso $(g=3)$

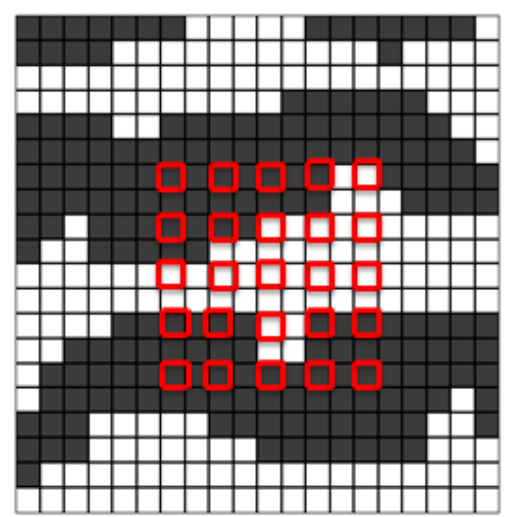

Grid média $(g=2)$

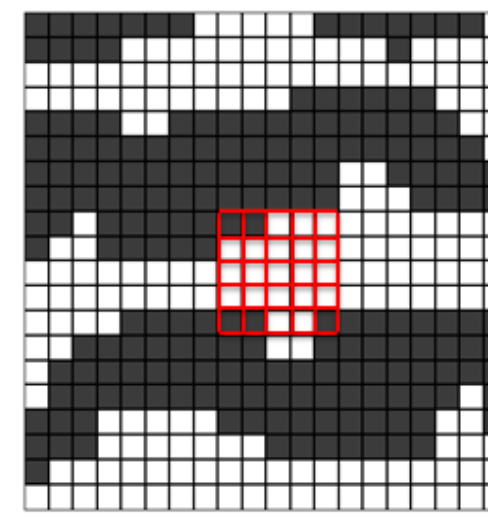

Grid fino $(g=1)$

Figura 2.16 llustração de uma $\mathbf{T I}$, usando três multi-grids de tamanho $\mathbf{5} \times \mathbf{5}$.

Semelhante à notação anterior, um evento de dados grossos é denotado por $\boldsymbol{d e v}_{T} g(\boldsymbol{u})$. A medida de similaridade segue sendo a mesma, já que o cálculo da similaridade é independente da grade (ARPAT, 2005). A aplicação do método de multi-grid no algoritmo é resumida no Quadro 2.4.

Quadro 2.4 Resumo da aplicação do método multi-grid.

\section{Simulação com Multi-grid}

1. Antes da simulação, o método de multi-grid começa com a construção do banco de dados de padrões mais grossos utilizando um template $\boldsymbol{T}^{\boldsymbol{g}=\boldsymbol{n}_{\boldsymbol{g}}}$.

2. A primeira simulação usa a grade da realização mais grossa $\boldsymbol{G}_{\boldsymbol{r} \boldsymbol{e}}^{\boldsymbol{g = \boldsymbol { n } _ { \boldsymbol { g } }}}$, a qual pode ser uma simulação condicional ou não condicional.

3. Utilizando $\boldsymbol{T}^{\boldsymbol{g}}$ execute, uma simulação de malha única em $\boldsymbol{G}_{\boldsymbol{r e}}^{\boldsymbol{g}}$ da realização re.

4. Depois de que a realização mais grossa é totalmente informada, $\boldsymbol{g}=\boldsymbol{g}-$ 1 o processo é repetido até concluir a simulação na malha mais fina $G_{r e}^{1}=G_{r e}$. 
Segundo Arpat (2005), o algoritmo não "congela" os valores que pertencem às malhas mais grossas, isto é unicamente realizado quando são transferidos a uma malha mais fina, ou seja, os valores ou nós a serem simulados ainda podem ser visitados pelo algoritmo durante seu caminhamento aleatório para $g=1$.

\subsection{Entropia}

O tamanho do template deve ser pequeno o suficiente para permitir flexibilidade durante a simulação e obter estatísticas significativas, porém não deve ser muito pequeno, porque isso restringe as reproduções de padrões maiores e não é capaz de capturar as variações a grande escala (HONARKHAH, 2011). Por outro lado, deve ser o suficientemente grande para representar as características reais presentes em uma imagem de treinamento, de modo a permitir uma simulação rápida.

Arpat e Honarkhah (2005 e 2011) apresentaram um método para deduzir o tamanho ótimo do template, baseado na informação da entropia dos padrões com diferentes tamanhos de template. Para selecionar o tamanho apropriado a entropia de Shannon é calculada conforme a equação 2.11. A entropia é uma medida estatística de aleatoriedade, que pode ser utilizada para caracterizar a textura da imagem de treinamento (HONARKHAH, 2011).

$$
H=\sum_{i=1}^{k} p_{i} \log \left(p_{i}\right)
$$

onde, $\boldsymbol{k}$ é o número de resultados possíveis da variável aleatória, e $\boldsymbol{p}_{\boldsymbol{i}}$ refere-se à função de massa de probabilidade, em outras palavras, representam o histograma. Quando há alta entropia, esta é ligada à elevada aleatoriedade. De acordo com o aumento no tamanho do template, a entropia dos padrões de 
uma imagem de treinamento é cada vez maior, quando o tamanho ótimo é obtido a entropia torna-se relativamente constante (HONARKHAH, 2011).

Segundo Honarkhah (2011), no caso bidimensional, a seleção do template ótimo começa com o pré-processamento da $\boldsymbol{T I}$, com vários tamanhos de $\boldsymbol{T}$, tem que se notar que seu tamanho deve ser definido com nós ímpares, $\boldsymbol{T}_{s}=\left\{(\mathbf{3} \times \mathbf{3}),(\mathbf{5} \times \mathbf{5}),(\mathbf{7} \times \mathbf{7}), \ldots,\left(\boldsymbol{n}_{x}^{\prime} \times \boldsymbol{n}_{y}^{\prime}\right)\right\}, \quad$ onde $\boldsymbol{T}_{s}$ é oconjunto dos diferentes tamanhos do template e $\boldsymbol{n}_{x}^{\prime} \boldsymbol{n}_{y}^{\prime}$ são as dimensões do template nas direções $x$ e $y$, respectivamente.

Calcula-se com a equação 2.12 a entropia média (ME) em um processo $2 D$, para um template $\boldsymbol{T}_{\boldsymbol{s}}$ (HONARKHAH, 2011).

$$
M E=\frac{1}{n_{T_{s}}}=\sum_{i=1}^{n_{T_{s}}} H\left(p a t_{T_{S}}^{k}\right)
$$

Onde $\boldsymbol{n}_{T_{s}}$ é o número de padrões que podem ser extraídos com um template de dimensões específicas $\boldsymbol{n}_{\boldsymbol{x}}^{\prime}, \boldsymbol{n}_{\boldsymbol{y}}^{\prime}$ e $\boldsymbol{p} \boldsymbol{a} \boldsymbol{t}_{\boldsymbol{T}_{s}}^{k}$ é o vetor de múltiplos pontos do conjunto de tamanho igual a $\boldsymbol{T}_{\boldsymbol{s}}$. Segundo Honarkhah (2011), a entropia da informação aumenta rapidamente ao longo das primeiras dimensões do template e à medida que $\boldsymbol{n}_{\boldsymbol{T}_{s}}$ se aproxima do tamanho "ótimo" ela começa a diminuir. Dessa forma o ponto de inflexão corresponde ao tamanho "ótimo" do template, que é obtido estatisticamente usando a máxima verossimilhança, juntamente com a segunda derivada da curva de entropia: $\boldsymbol{f}_{\boldsymbol{i}}^{\prime \prime}=\boldsymbol{f}_{\boldsymbol{i}+\mathbf{2}}-\mathbf{2} \boldsymbol{f}_{\boldsymbol{i}+\mathbf{1}}+\boldsymbol{f}_{\boldsymbol{i}}$ onde $\boldsymbol{f}$ é a função objetivo e sua igualdade é dada pela fórmula das diferenças finitas (HONARKHAH \& CAERS, 2010). Segundo Honarkhah (2011), a abordagem anterior pode ser resumida conforme apresentado no quadro 2.5 . 
Quadro 2.5 O algoritmo de entropia de Shannon para obter o $\boldsymbol{T}$ "ótimo".

\section{Algoritmo de Entropia}

1. Requer uma imagem de treinamento $\{\boldsymbol{T I}(\boldsymbol{n} \boldsymbol{x} \times \boldsymbol{n} \boldsymbol{y})\}$

2. Tamanhos do template previamente selecionados $\boldsymbol{n}_{x}^{\prime}, \boldsymbol{n}_{\boldsymbol{y}}^{\prime}$

3. Tamanho máximo do template $\boldsymbol{T}_{s}=\left\{(\mathbf{3} \times \mathbf{3}),(\mathbf{5} \times \mathbf{5}), \ldots,\left(\boldsymbol{n}_{x}^{\prime} \times \boldsymbol{n}_{y}^{\prime}\right)\right\}$

4. Construa a base de padrões: $\boldsymbol{p a t d}_{T}$

5. $E_{i}=E_{i+2}-2 E_{i+1}+E_{i}$

6. $\quad \boldsymbol{P} \leftarrow$ Máxima verossimilhança lognormal da curva de entropia $\boldsymbol{E}_{1:\left(n_{T_{s}}-2\right)}$

7. Finalmente o template "ótimo" $(\boldsymbol{P} \times \boldsymbol{P})$

Para imagens de treinamento no espaço tridimensional, o mesmo algoritmo pode ser executado, no entanto, a diferença do caso bidimensional, também deve ser calculado o tamanho do template na direção vertical (HONARKHAH, 2011). De acordo com Honarkhah (2011), as dimensões são analisadas de forma independente, as dimensões do template no plano horizontal são consideradas simultaneamente à direção vertical. Finalmente, os dois resultados são combinados para estabelecer o template ótimo.

O exemplo da Figura 2.17 mostra uma imagem de treinamento $2 D$ binária com dimensões de $111 \times 111$ e representa canais sinuosos.

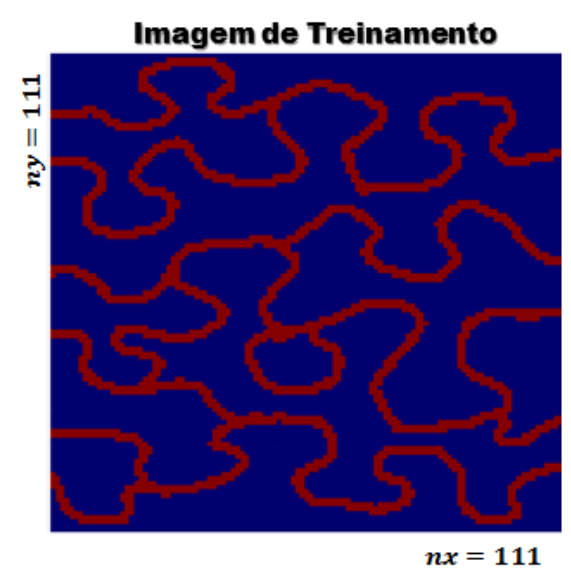

Figura 2.17 Imagem de treinamento 2D, representando canais sinuosos (Fonte: MARIETHOZ E CAERS, 2014). 
Observa-se na Figura 2.18 que, para obter o tamanho "ótimo" de template para a TI da Figura 2.17, define-se uma sequência de números ímpares, com valor máximo igual a 33. Ilustra-se na Figura $2.18 a$ a entropia média $(\boldsymbol{M E})$ em função do tamanho de $\boldsymbol{T}$. A $(\boldsymbol{M E})$ é obtida a partir da média das entropias de todos os padrões contidos em todos os cortes de TI. $\mathrm{Na}$ Figura $2.18 b$ mostra-se o perfil de probabilidade dessa curva, indicando que 0 template "ótimo" é igual a $13 \times 13$.
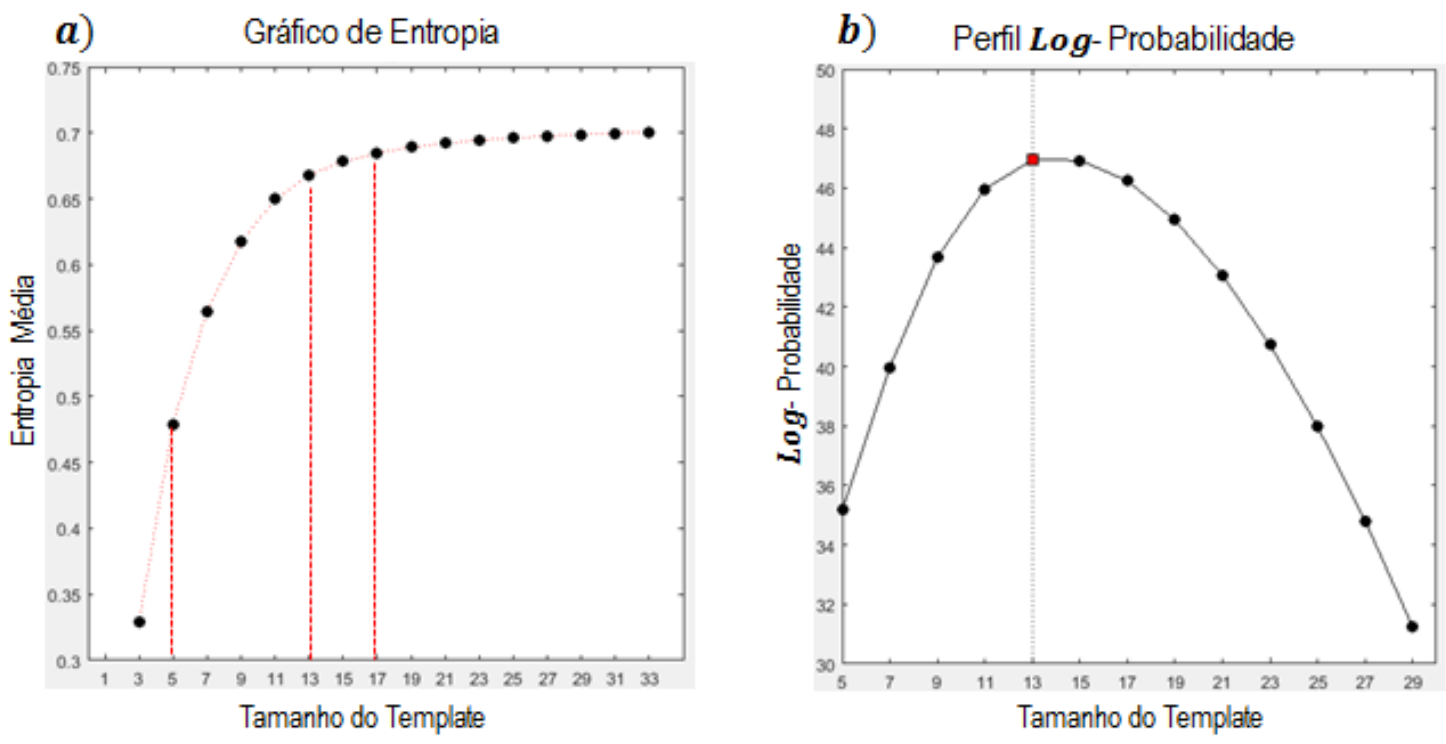

Figura 2.18 A curva de entropia média (Fig. 2.18a) e a probabilidade de registro do perfil que indica que o melhor template possui um tamanho de $13 \times 13$ (Fig.2.18b). 


\subsection{Conectividade}

A análise de conectividade é importante quando se deseja medir ou quantificar as interconexões entre células de uma mesma litologia, no nosso caso a conectividade de canais ou categorias reproduzidas pelo MPS.

A função de conectividade $\boldsymbol{\tau}(\boldsymbol{h})$, é definida como a probabilidade de que duas células $\boldsymbol{x}$ e $\boldsymbol{x}^{\prime}$ de $\boldsymbol{A}$ estejam conectados: (ALLARD, 1993).

$$
\tau\left(x, x^{\prime}\right)=P\left(x \Leftrightarrow x^{\prime} \mid x, x^{\prime} \in A\right)
$$

Quando o conjunto aleatório $A$ é estacionário, assume-se que a função para um vetor de distância $\boldsymbol{h}$ é dada por:

$$
\tau(h)=P\left(x \Leftrightarrow x^{\prime}+h \mid x, x^{\prime}+h \in A\right)
$$

Onde $\boldsymbol{\tau}(\mathbf{0})=1$ e $\boldsymbol{\tau}(\boldsymbol{h})$ é uma função decrescente à medida que $\boldsymbol{h}$ aumenta.

\subsubsection{Descrição da Função CONNEC3D}

Neste trabalho foi utilizada a função CONNEC3D. Segundo PardoIguzquiza \& Dowd, 2003, a função contem nove linhas que descrevem os parâmetros dos dados de entrada (quadro 2.6).

1) A primeira linha deve ser 0 ou 1 de acordo com a fase a ser analisada.

2) $\mathrm{Na}$ segunda linha, existem 3 opções, 6 conectividade de face, 18 conectividade de borda e 26 conectividade de vértice.

3) Especifica o nome do arquivo.

4) A quarta linha representa os números de blocos $\boldsymbol{X}, \boldsymbol{Y}$ e $\boldsymbol{Z}$.

5) A quinta linha especifica as dimensões do bloco da malha.

6) A sexta linha fornece o número de passos para estimar a função de conectividade. 
7) Finalmente, as últimas 3 linhas representam os nomes dos três arquivos de saída para as estatísticas de conectividade

Quadro 2.6 Dados de entrada para a função CONNEC3D.

\begin{tabular}{|c|c|}
\hline \multicolumn{2}{|c|}{ Função CONNEC3D } \\
\hline 1 & Fase para analisar 0 ou 1 \\
\hline 6 & 6, 18 ou 26 -conectividade \\
\hline Nome.dat & Mapa do indicador $(0 / 1)$ \\
\hline 1011011 & $N X N Y$ e $N Z$ \\
\hline $\begin{array}{lll}1.0 & 1.0 \quad 1.0\end{array}$ & $D X D Y$ e $D Z$ \\
\hline 100 & Número de passos para função de conectividade \\
\hline Nome.STA & Estatísticas de conectividade de arquivos de saída \\
\hline Nome.CCO & Componentes conectados do arquivo de saída \\
\hline Nome.COF & Função de conectividade de arquivo de saída \\
\hline
\end{tabular}

\subsection{Escalonamento Multidimensional}

O MDS é uma técnica utilizada para explorar a estrutura dos dados (ou seja, semelhanças ou distâncias). O MDS consiste na representação visual de um conjunto de objetos como pontos em um espaço multidimensional, ou seja, pontos correspondentes a objetos semelhantes estão localizados juntos, enquanto os objetos diferentes estão localizados distantes (CAERS, 2011). 


\section{MATERIAIS E MÉTODOS}

\subsection{Materiais}

Nesta seção são apresentados os dados sintéticos utilizados nas simulações, estes fenômenos espaciais foram escolhidos por serem amplamente utilizados nos MPS para testar os diferentes algoritmos computacionais. Tambem são mostrados os parâmetros que foram utilizados.

\subsubsection{Banco de Dados}

Para testar as diferentes distâncias de similaridade na simulação baseada em padrões, como já mencionado, foram utilizados quatro bancos de dados sintéticos: três bidimensionais e um tridimensional. As Figuras 3.1 e 3.2 ilustram os bancos de dados sintéticos.

O primeiro banco de dados (Figura 3.1a) é uma TI de canais sinusoidais apresentados por Strebelle (2002) e semelhante à TI mostrada na Figura 2.17, porém com dimensões reduzidas a $\mathbf{1 0 1} \times \mathbf{1 0 1}$ totalizando 10.201 células. O segundo conjunto de dados de variáveis categóricas representa um depósito fluvial com quatro categorias: folhelho, areia, barra de meandro (que será referida apenas como barra) e espraiamento de crevasse (crevasse splay), referida apenas como crevasse (Figura 3.1b), essa TI é composta por 101 células nas direções $\boldsymbol{x}$ e $\boldsymbol{y}$. Ao contrário das $\boldsymbol{T I}$ anteriores, o terceiro banco de dados (Figura 3.1c) é uma variável contínua de uma parede de pedra, cujos valores foram normalizados ou transformados, o que implica um reescalonamento dos valores no intervalo de $[\mathbf{0 , 1}]$. Esta $\boldsymbol{T I}$ tem um tamanho de $200 \times 200($ ZHANG, 2006). Finalmente a Figura 3.2 é uma TI binária $3 D$ de areia / folhelho com dimensões de $69 \times 69 \times 39$ nas direções $X, Y$ e $\boldsymbol{Z}$ respectivamente (HONARKHAH \& CAERS, 2010).

Todas as células das $\boldsymbol{T I}$ selecionadas têm dimensões de $\mathbf{1 m} \times \mathbf{1} \boldsymbol{m}$. Apresentam-se nas Tabelas 3.1,3.2,3.3 e 3.4 as estatísticas básicas de cada TI utilizada. 
a)

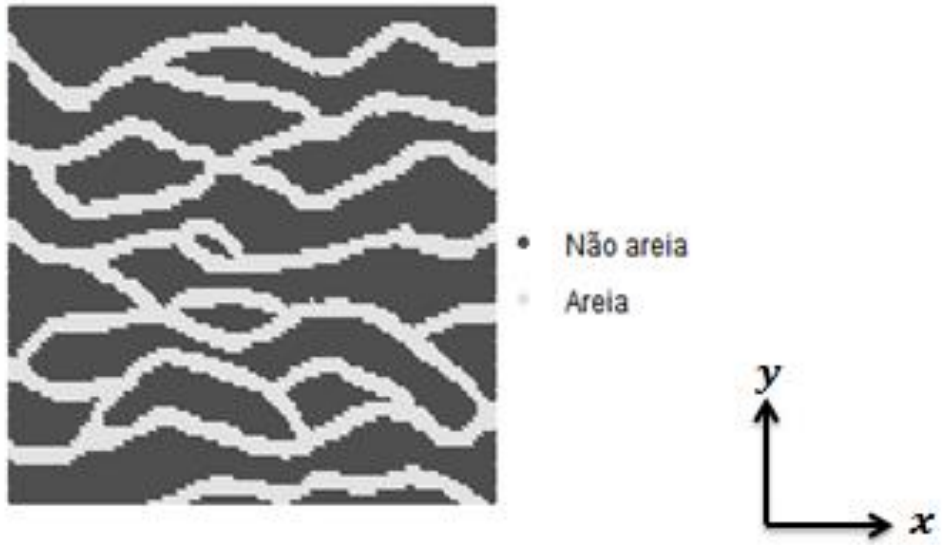

b)

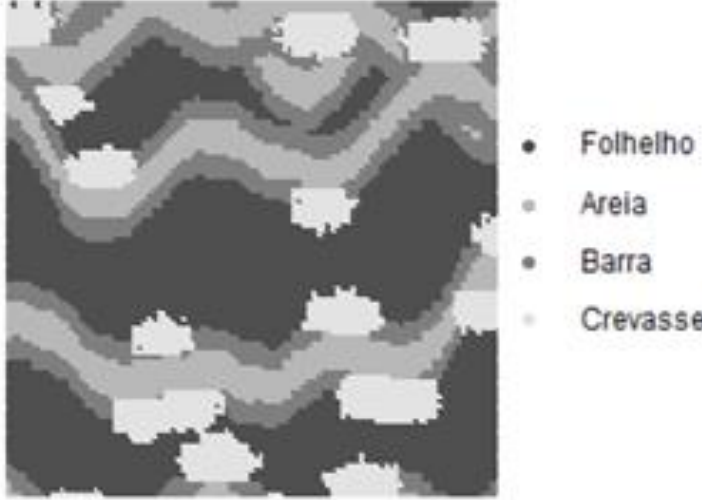

c)

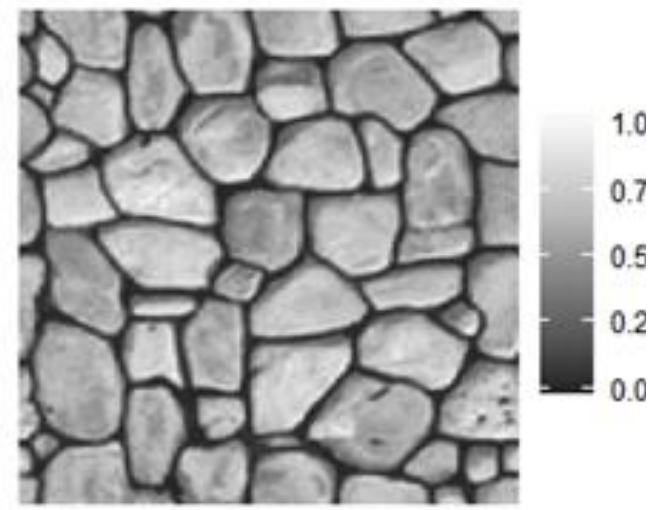

Figura 3.1 Imagens de treinamento. (a) Imagem de treinamento do canal (STREBELLE 2002), (b) TI de uma variável categórica, deposição de rios com quatro categorias (HONARKHAH \& CAERS, 2010), (c) Imagem de treinamento de muro de pedra (ZHANG, 2006).

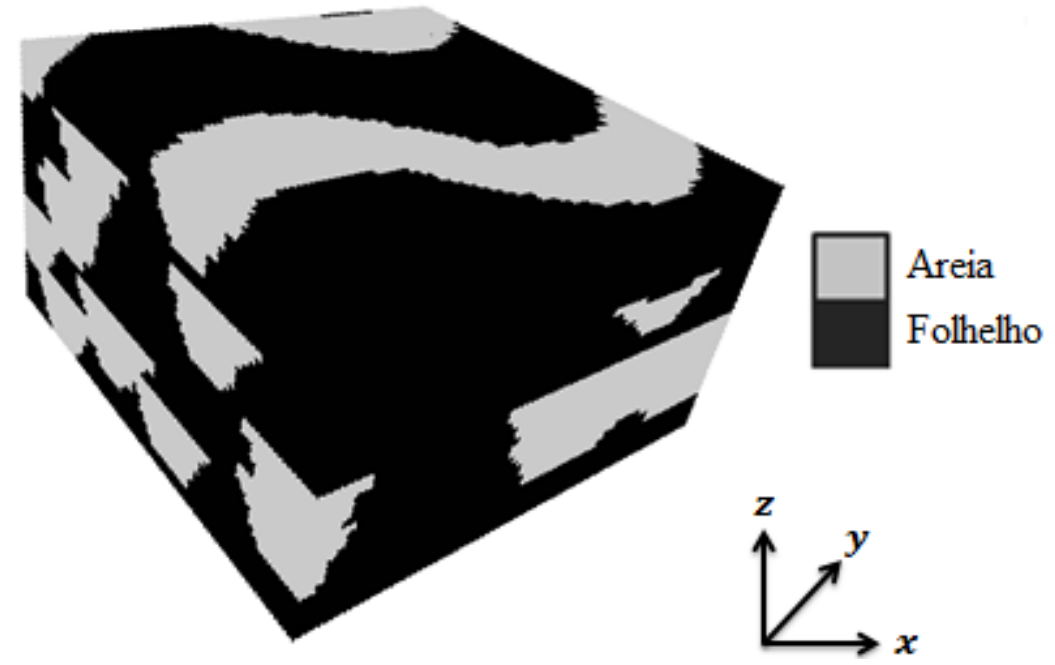

Figura 3.2 TI binaria em 3D (Fonte: HONARKHAH \& CAERS, 2010). 
Tabela 3.1 Proporção do tipo de litologia a partir da $\boldsymbol{T I}$ do canal (Primeira $\boldsymbol{T I}$ ).

\begin{tabular}{ccc}
\hline Litologia & Número de células & Proporção da amostra \\
\hline Areia & 3315 & 0.324 \\
Não Areia & 6886 & 0.675 \\
\hline
\end{tabular}

Tabela 3.2 Proporção do tipo de litologia a partir da $\boldsymbol{T I}$ do canal (Segunda $\boldsymbol{T I}$ ).

\begin{tabular}{lcc}
\hline Categorias & Número de células & Proporção da amostra \\
\hline Folhelho & 4580 & 0.448 \\
Areia & 2054 & 0.201 \\
Barra & 2043 & 0.200 \\
Crevasse & 1524 & 0.149 \\
\hline
\end{tabular}

Tabela 3.3 Resumo estatístico da terceira $\mathbf{T I}$ do muro de pedra (Terceira $\boldsymbol{T I}$ ).

\begin{tabular}{cccccc}
\hline Mínimo & 1ㅇo Quartil $^{\text {Mediana }}$ & Média & 3 Quartil & Máximo \\
\hline 0.000 & 0.341 & 0.576 & 0.501 & 0.674 & 1.000 \\
\hline
\end{tabular}

Tabela 3.4 Proporção do tipo de litologia a partir da TI em 3D.

\begin{tabular}{ccc}
\hline Litologia & Número de células & Proporção da amostra \\
\hline Areia & 77202 & 0.415 \\
Folhelho & 108477 & 0.584 \\
\hline
\end{tabular}




\subsubsection{Dados de Condicionamento}

Para cada um dos modelos sintéticos $1 \%$ da população de cada TI foi extraída aleatoriamente aplicando-se o método de amostragem aleatória simples, os resultados destas amostragens foram utilizados como dados condicionantes. Ressalta-se que o algoritmo SIMDISPAT possui a capacidade de gerar simulações sem a necessidade do uso de dados condicionantes, denominado simulação não condicional. Os resultados da amostragem espacial estão ilustrados nas Figuras 3.3 e 3.4. Para cada conjunto de amostras, foram obtidas estatísticas descritivas que são apresentadas nas Tabelas 3.5, 3.6, 3.7 e 3.8 respectivamente.

a)

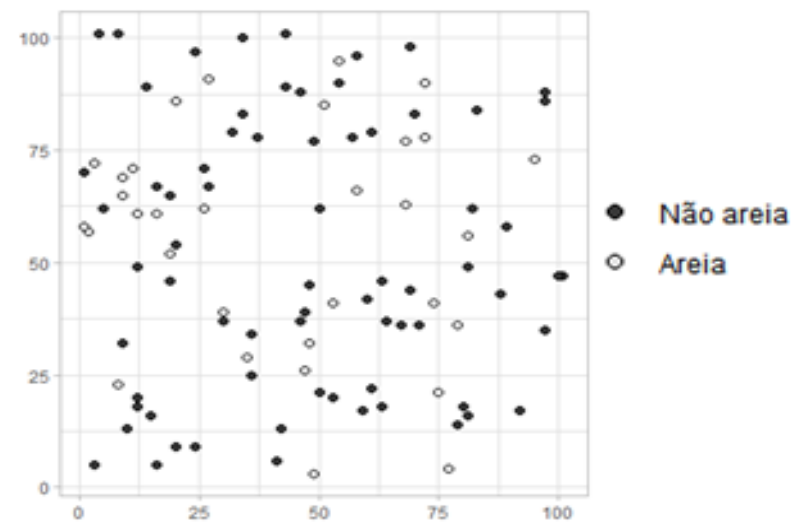

b)
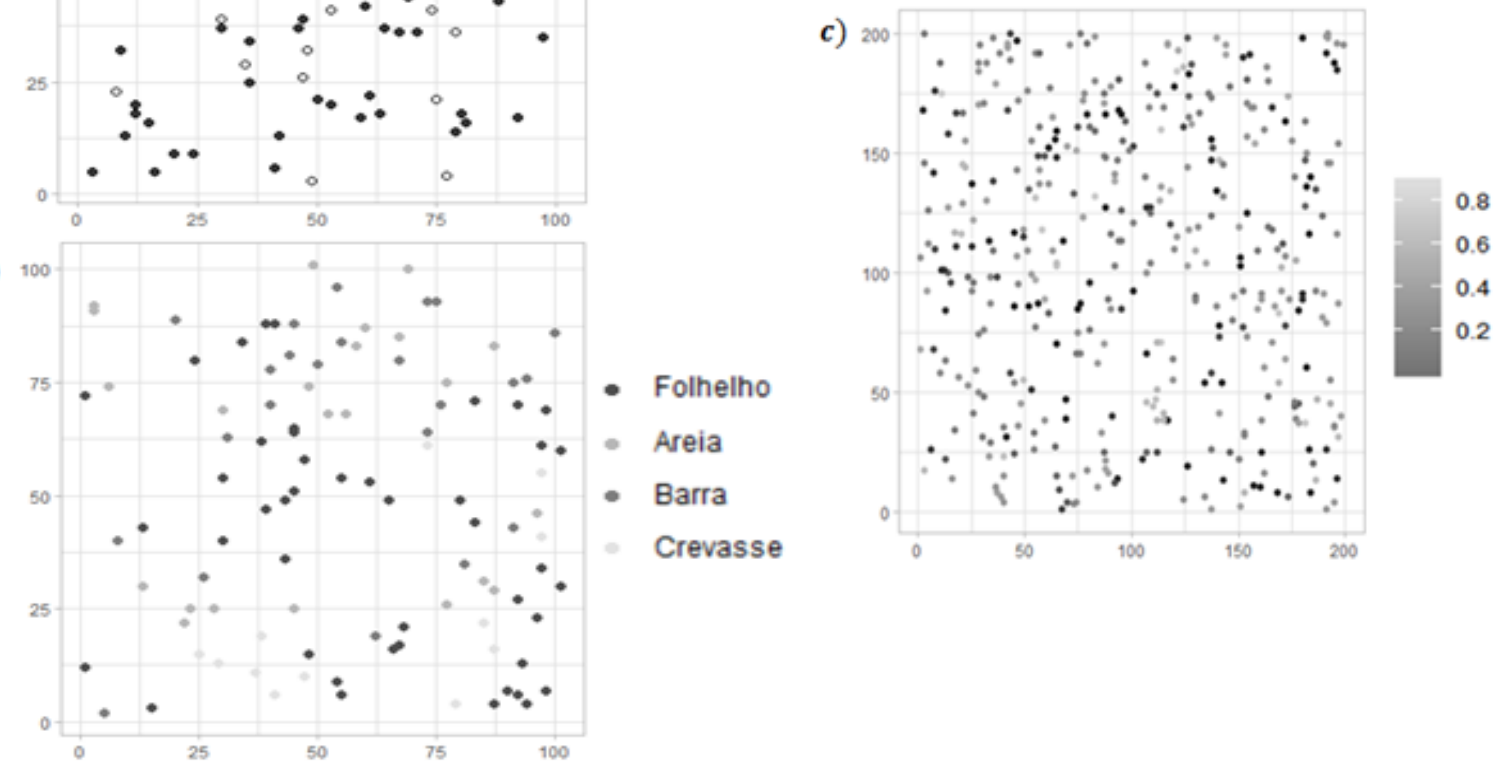

Figura 3.3 Mapa de localização das amostras selecionadas pelo método da amostragem simples (porcentagem de 1\%). (a) Primeira amostra do Banco de dados do canal (b) Segunda amostra do banco de dados de deposição fluvial com quatro categorias e (c) Terceira amostra do banco de dados de muro de pedra. 


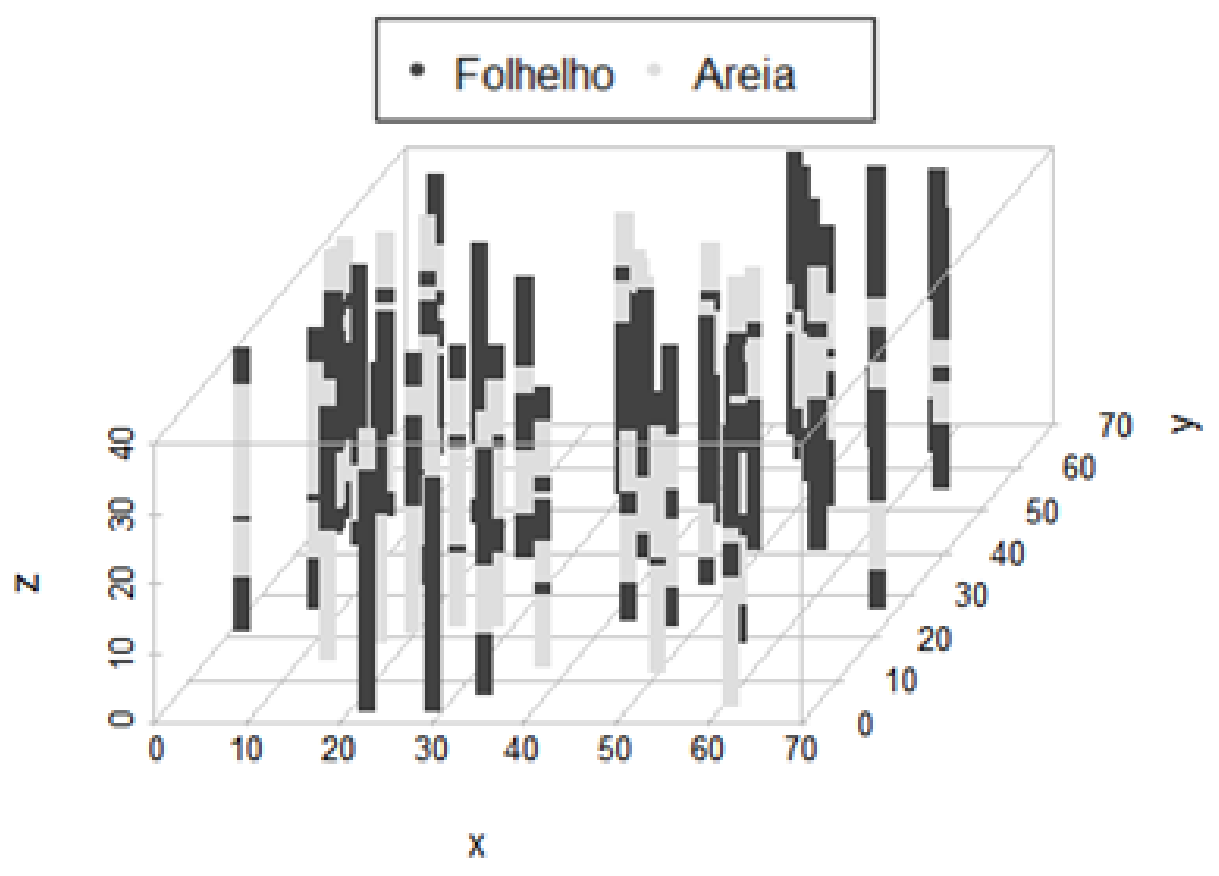

Figura 3.4 Distribuição espacial dos dados condicionantes pelo método de amostragem simples, para o banco de dados em $3 D$.

Tabela 3.5 Proporção do tipo de litologia a partir dos dados amostrados (Primeira amostra do Banco de dados do canal binário 2D).

\begin{tabular}{ccc}
\hline Litologia & Número de células & Proporção da amostra \\
\hline Areia & 41 & 0.401 \\
Não Areia & 61 & 0.598 \\
\hline
\end{tabular}

Tabela 3.6 Proporção do tipo de litologia a partir dos dados amostrados (segunda amostra do banco de dados).

Categorias Número de células Proporção da amostra

Folhelho

Areia

Barra 
Tabela 3.7 Resumo das estatísticas descritivas da terceira amostra do banco de dados de muro de pedra

\begin{tabular}{cccccc}
\hline Mínimo & 1 Quartil & Mediana & Média & 3 Quartil & Máximo \\
\hline 0.003 & 0.317 & 0.572 & 0.491 & 0.670 & 0.882 \\
\hline
\end{tabular}

Tabela 3.8 Proporção do tipo de litologia a partir do banco de dados em $\mathbf{3 D}$.

\begin{tabular}{ccc}
\hline Litologia & Número de células & Proporção da amostra \\
\hline Areia & 822 & 0.446 \\
Folhelho & 1017 & 0.553 \\
\hline
\end{tabular}

\subsubsection{Parâmetros a Considerar}

Observam-se na Tabela 3.9 os parâmetros usados nas simulações, para cada uma das bases de dados empregadas. Onde BD1 é o banco de dados binários $2 D, \boldsymbol{B D} 2$ representa o banco de dados da variável categórica do depósito fluvial das quatro categorias, BD3 mostra o banco de dados de muro de pedra, finalmente $\boldsymbol{B D} \mathbf{4}$ é o banco de dados em $3 D$.

Tabela 3.9 Parâmetros usados no algoritmo SIMDISPAT, para gerar as diferentes simulações.

\begin{tabular}{lcccc}
\hline \multicolumn{1}{c}{ PARÂMETROS } & BD1 & BD2 & BD3 & BD4 (3D) \\
\hline Dimensão da malha $x, y$ e $z$ & $101 \times 101$ & $101 \times 101$ & $200 \times 200$ & $69 \times 69 \times 39$ \\
Tamanho do template & $15 \times 15 \times 1$ & $19 \times 19 \times 1$ & $21 \times 21 \times 1$ & $17 \times 17 \times 13$ \\
Tamanho do Inner patch & $9 \times 9 \times 1$ & $9 \times 9 \times 1$ & $15 \times 15 \times 1$ & $11 \times 11 \times 9$ \\
Tolerância de partição de $\boldsymbol{B D}$ & 1000 & 1000 & 1000 & 10000 \\
Porcentagem do $\boldsymbol{B D}$ & $0.70 \%$ & $0.70 \%$ & $0.70 \%$ & $0.80 \%$ \\
Número de múltiplas malhas & 3 & 3 & 3 & 3 \\
Número de simulações & 100 & 100 & 100 & 100 \\
\hline
\end{tabular}




\section{- Tamanho do template}

Conforme descrito na seção 2.9, o tamanho ótimo do template, foi selecionado para cada conjunto de dados, com base nas informações de entropia de Shannon. Deve-se mencionar que as dimensões do template são do mesmo tamanho tanto para escanear a $\boldsymbol{T I}$, quanto para as realizações re. Na Figura 3.5 mostram-se os resultados de entropia média e o perfil de probabilidade que indicam o tamanho ótimo do $\boldsymbol{T}$ para cada banco de dados bidimensional.

\section{- Tolerância e porcentagem do banco de dados}

Esses dois parâmetros do SIMDISPAT ajudam a otimizar o tempo computacional e melhorar a qualidade do resultado, principalmente quando há uma grande quantidade de dados.

\section{- Número de múltiplas malhas}

Este parâmetro foi selecionado de acordo com Dujardin (2007): nmult = 3, dado que, a utilização de um nmult $=1$ não reproduz as estruturas em grande escala da TI. E um $\boldsymbol{n m u l t}=\mathbf{2}$ não caracteriza bem a continuidade espacial das estruturas, enquanto a $\mathbf{n m u l t}=\mathbf{3}$ as realizações têm uma melhor coincidência com a $\boldsymbol{T I}$.

\section{- Número de simulações}

Decidiu-se utilizar cem realizações, devido a que na literatura este valor é amplamente utilizado, além de ser um valor suficiente para capturar a variabilidade do fenômeno.

\section{- Pesos para dados de condicionamento}

Especificando os pesos para cada tipo de nó: $\boldsymbol{\omega}_{\mathbf{1}}=\mathbf{0 . 7}$ dados condicionantes, $\boldsymbol{\omega}_{2}=\mathbf{0 . 2}$ nós previamente simulados, $\boldsymbol{\omega}_{3}=\mathbf{0 . 1}$ nós informados. 

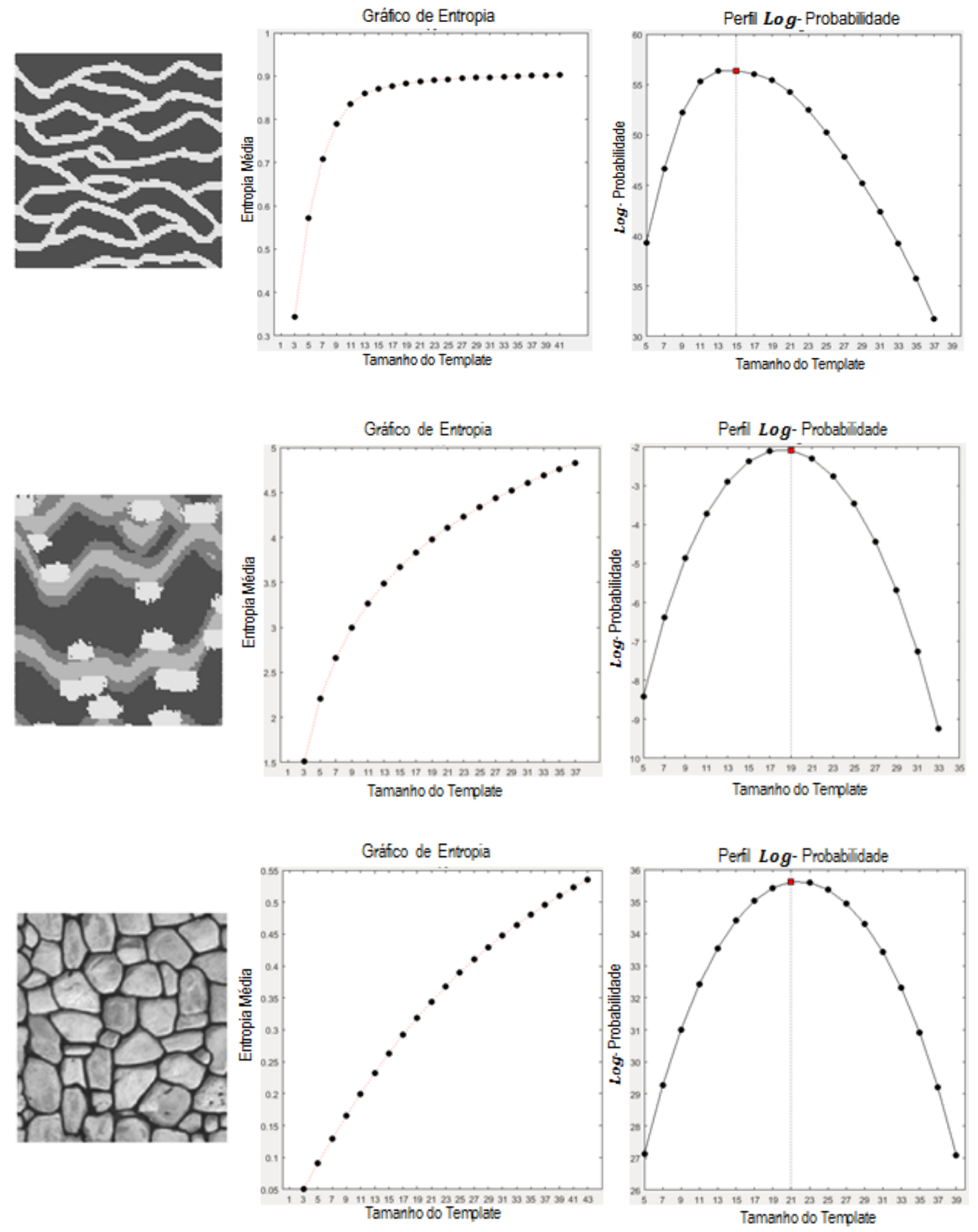

Figura 3.5 A entropia de Shannon gera o tamanho do ótimo do template para cada banco de dados 2D. A Figura mostra a TI (esquerda), a curva de entropia média (meio) e o perfil de probabilidade (direita). 


\subsubsection{Parâmetros da Conectividade}

Mostram-se na Tabela 3.10 os parâmetros que foram utilizados para a análise de conectividade.

Tabela 3.10 Parâmetros utilizados na função CONNEC3D para a análises de conectividade.

$\begin{array}{llll}\text { PARÂMETROS } & \text { BD1 } & \text { BD2 } & \text { BD4 (3D) }\end{array}$

\begin{tabular}{lccc} 
Fase para Analisar & 1 & 1 & 1 \\
Conectividade & 6 & 6 & 6 \\
Número de blocos $\boldsymbol{X}, \boldsymbol{Y}$ e $\boldsymbol{Z}$. & $101 \times 101$ & $101 \times 101$ & $69 \times 69 \times 39$ \\
Dimensões do bloco & 1.0 & 1.0 & 1.0 \\
Número de Passos & 100 & 100 & 70 \\
\hline
\end{tabular}

\subsection{Métodos}

Esta seção tem como objetivo mostrar o método de simulação que foi desenvolvido para a modelagem de MPS, denominado SIMDISPAT.

O algoritmo da SIMDISPAT foi baseado nos códigos fontes da simulação SNESIM desenvolvida por Strebelle (2002) e o proposto por Arpat (2005), para o desenvolvimento da SIMPAT. O algoritmo SIMDISPAT foi desenvolvido em $R$ (R CORE TEAM, 2019), além disso, Matlab 2015 foi usado para calcular a entropia de Shannon.

No algoritmo SIMDISPAT, a função PatternBase descrita na seção 2.4 foi modificada (Apêndice C). Além disso, foi criada uma função chamada CoordTemplate (Apêndice B), com o objetivo de fazer de forma simples 0 Template e o inner patch, os quais podem ser em $2 D$ ou $3 D$. A função CoordTemplate está resumida no quadro 3.1 . 
Quadro 3.1 Descrição da função CoordTemplate do algortimo SIMDISPAT.

\section{Função CoordTemplate}

1. Para gerar o modelo são usados três argumentos $\boldsymbol{n x} \boldsymbol{T}, \boldsymbol{n y} \boldsymbol{T}, \boldsymbol{n z} \boldsymbol{T}$, os quais representam as dimensões do $\boldsymbol{T}$, o qual é utilizado, para escanear a $\boldsymbol{T I}$ e, assim, obter todos seus vetores $\boldsymbol{t i}_{\boldsymbol{T}}(\boldsymbol{u})$. Por outro lado, inxT, inyT e inzT representam as dimensões do inner patch. No entanto, o template como o inner patch são utilizados na simulação, embora os valores internos $\boldsymbol{i} \boldsymbol{p}_{\boldsymbol{T}}(\boldsymbol{u})$ estejam congelados.

2. Os valores retornados pela função são as coordenadas do template e um vetor binário, de modo que os valores iguais a um representem o inner patch. Assim, os valores zero não são congelados e os valores iguais a um, são os nós que serão congelados pelo algoritmo nas realizações, como se mostra na Figura 3.6.

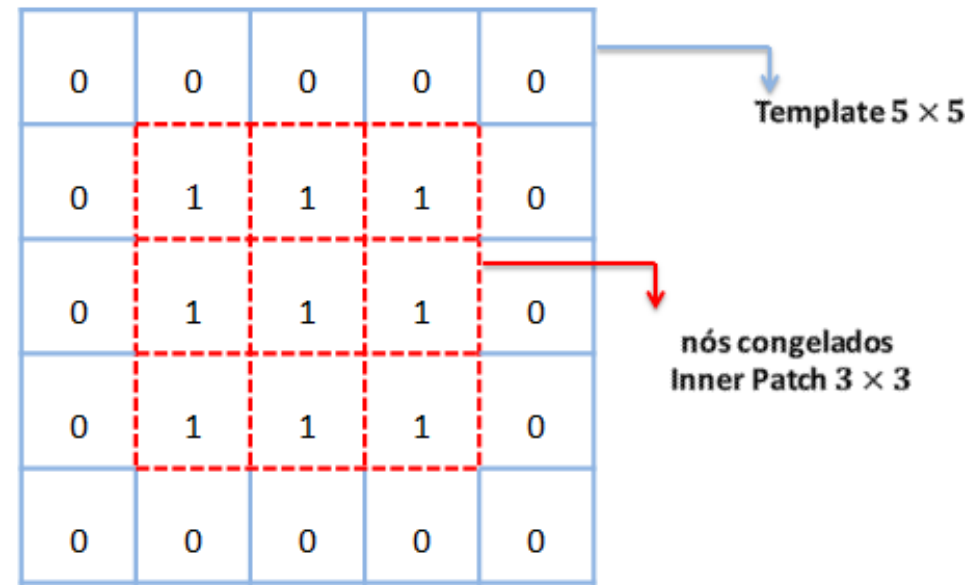

Figura 3.6 Resultado da função CoordTemplate. Valores iguais a um representam os nós congelados durante a simulação.

No algoritmo da SIMDISPAT a função PatternBase faz o préprocessamento da $\boldsymbol{T I}$ seguindo um caminhamento aleatório e , em seguida, armazena os padrões $\boldsymbol{p a t}_{T}^{k}$ como vetores $\boldsymbol{t i}_{\boldsymbol{T}}(\boldsymbol{u})$, o total dos padrões formados é chamado de $\boldsymbol{p a t d b}_{T}$ (banco de dados). No algoritmo SIMDISPAT a equação 3.1 representa o número total de padrões em uma imagem $2 D$ ou $3 D$. 


$$
n_{\text {pat }}=[n x-(n x T-1)] *[n y-(n y T-1)] *[n z-(n z T-1)]
$$

onde $\boldsymbol{n} \boldsymbol{x}, \boldsymbol{n y}, \boldsymbol{n z}$ são as dimensões da imagem de treinamento e $\boldsymbol{n} \boldsymbol{x} \boldsymbol{T}, \boldsymbol{n y} \boldsymbol{T}, \boldsymbol{n z} \boldsymbol{T}$ representam o tamanho do template.

SIMDISPAT dispõe de um argumento chamado patpercent, o qual tem por objetivo fornecer uma porcentagem, para assim extrair por amostragem aleatória simples sem repetição os padrões da TI. Para o exemplo ilustrado na Figura 3.7 um template bidimensional de $\mathbf{5} \times \mathbf{5}$ foi escolhido e um patpercent $=33 \%$, que para o número total de padrões da $\boldsymbol{T I}\left(\boldsymbol{p a t d b}_{T}=49\right)$ foram selecionados 16 padrões $\left(\boldsymbol{p a t d b}_{T}=\mathbf{1 6}\right)$.

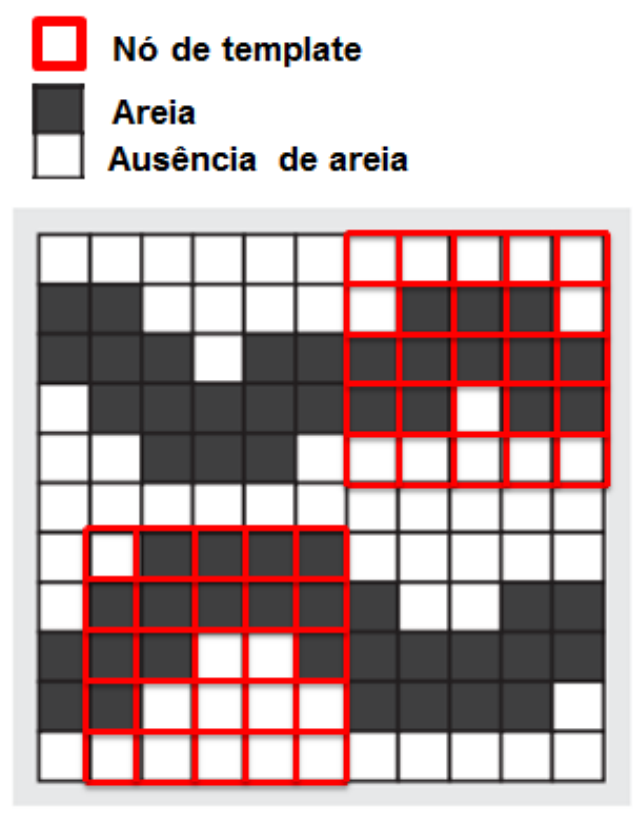

Imagem de treinamento

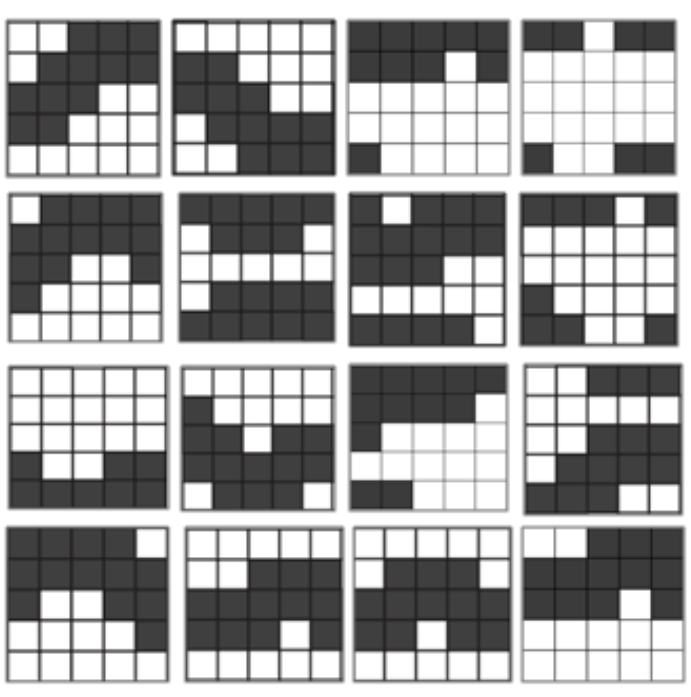

Banco de dados de padrões com um patpercent $=0.33$

Figura 3.7 Obtenção de padrões, através de amostragem simples, para a construção do banco de dados a partir de um template de tamanho $5 \times 5$ (Fonte: modificado de ARPAT, 2005).

Com o objetivo de evitar um alto custo computacional, como já mencionado, os padrões $\boldsymbol{p a t}_{T}^{k}$ são escolhidos aleatoriamente. Além disso, para o SIMDISPAT, o banco de dados obtido será particionado em listas. Para isso, a função PatternBase captura e armazena os diferentes padrões obtidos, aplicando os resultados da função CoordTemplate. A descrição da função, para obter o banco de dados de padrões da $\boldsymbol{T I}$, é fornecida no quadro 3.2: 
Quadro 3.2 Descrição da função PatternBase do algoritmo SIMDISPAT.

\section{Função PatternBase}

1. A função patternBase dispõe de argumentos diferentes, nos quais são definidas as dimensões da $\boldsymbol{T I}$ e do template $\boldsymbol{T}$, também são utilizados os argumentos para a expansão do template e da malha da realização $\boldsymbol{G}_{\boldsymbol{r} \boldsymbol{e}}$. Além disso, possui o argumento $\mathbf{d b t o l}$ que é usado para dividir patd $\boldsymbol{b}_{T}$ (por exemplo, se $\boldsymbol{d b t o l}=10, \boldsymbol{p a t d b _ { T }}=16$ e um $\boldsymbol{n}_{T}=\mathbf{2 5}$, duas listas serão criadas com matrizes de tamanhos iguais a $10 \times 25$ e $6 \times 25$. Os 10 primeiros padrões de, $\boldsymbol{p a t} t_{T}^{k}, \boldsymbol{k}=\mathbf{1}, \ldots, \mathbf{1 6}$ são separados na primeira lista e os 6 padrões restantes na segunda lista. Finalmente, o patpercent é o argumento que permite extrair uma porcentagem de padrões, de forma aleatória do banco de dados.

2. Finalmente, o valor obtido na função PatternBase é o banco de dados de padrões $\boldsymbol{p a t d}_{T}$ e, consequentemente, possui os padrões $\boldsymbol{p a t}_{T}^{k}, \boldsymbol{k}=$ $\mathbf{1}, \ldots, \boldsymbol{n}_{\text {pat }_{T}}$. Sendo que os padrões são organizados de acordo com a lista feita pelo $\mathbf{d b t o l}$.

A implementação do SIMDISPAT é mostrada na Figura 3.80 algoritmo começa com a função coordeTemplate, que cria um $\boldsymbol{T}$ e um $\boldsymbol{i p}_{T}(\boldsymbol{u})$. A função PatternBase constrói o patdb $\boldsymbol{~ p}_{T}$, com a ajuda de ScanTemplate cuja função é verificar se as coordenadas do $\boldsymbol{T}$ estão dentro da $\boldsymbol{T I}$. Caso o algoritmo contenha dados de condicionamento a função AssignData atribui os dados da amostra original aos nós mais próximos. A função Ordepath gera um caminho aleatório. No processo de simulação a função SimulationPath, que contém cinco funções internas: getDataEvent é a função que mapeia os diferentes estados atribuídos a um nó. AssignWeight Atribui os pesos. A função similarPattern e disDistance calculam o padrão mais semelhante utilizando uma das distancias. Finalmente a função pastePattern É a função que simula o nó central do $\boldsymbol{T}$, bem como aqueles que pertencem ao $\boldsymbol{i} \boldsymbol{p}_{T}(\boldsymbol{u})$, além disso, esta função congela os nós em questão. 


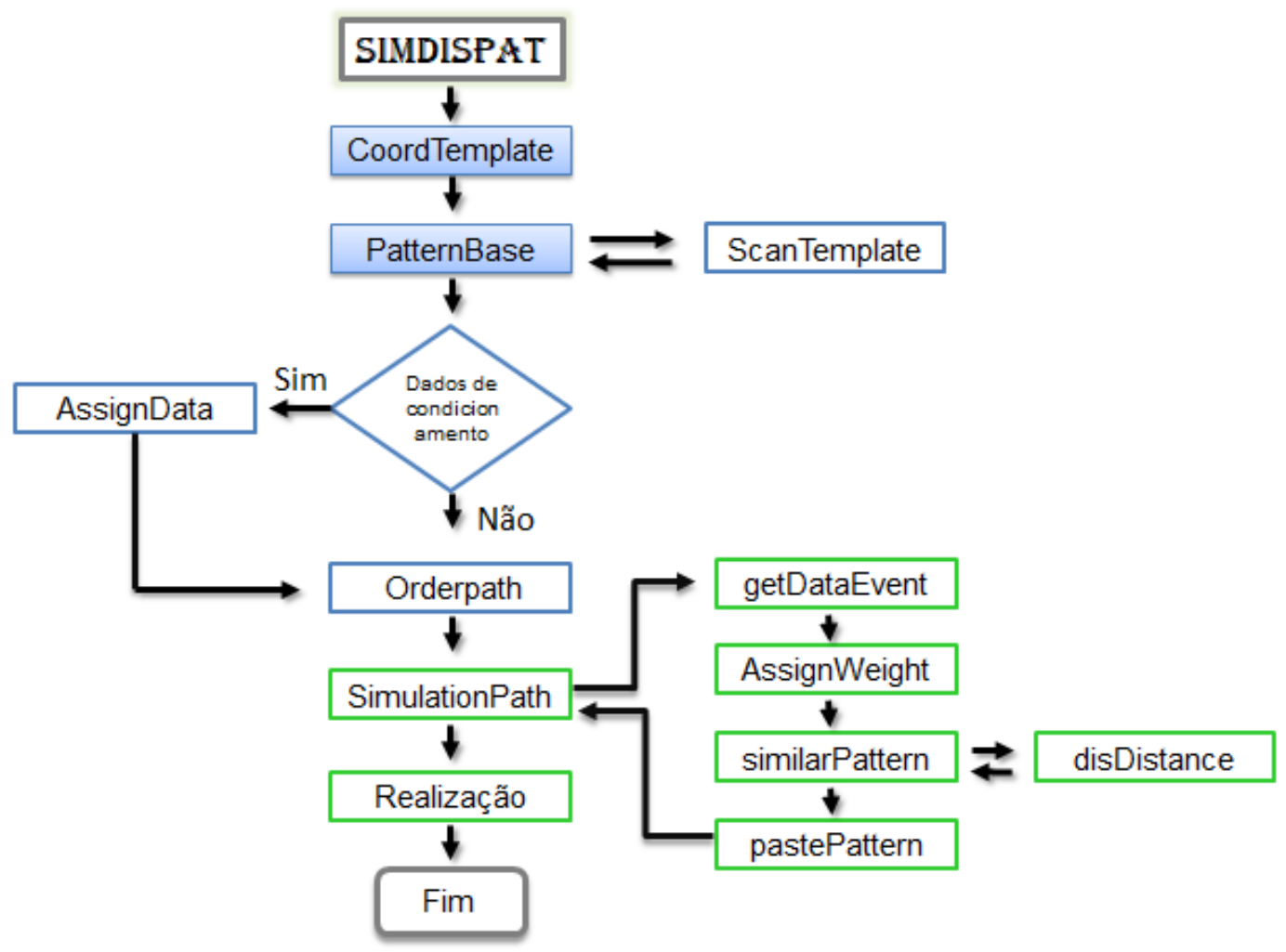

Figura 3.8 Fluxograma do algoritmo SIMDISPAT.

Para validar e comparar os resultados das realizações simuladas, obtidos pelo SIMDISPAT foi criada uma função em $R$ onde a função CONNEC3D é executada para cada uma das simulações obtidas para cada uma das distâncias, a partir desse resultado é originada a matriz de conectividade a partir da qual a matriz média foi calculada e que resultou no gráfico de conectividade, conforme apresentado na figura 3.4.

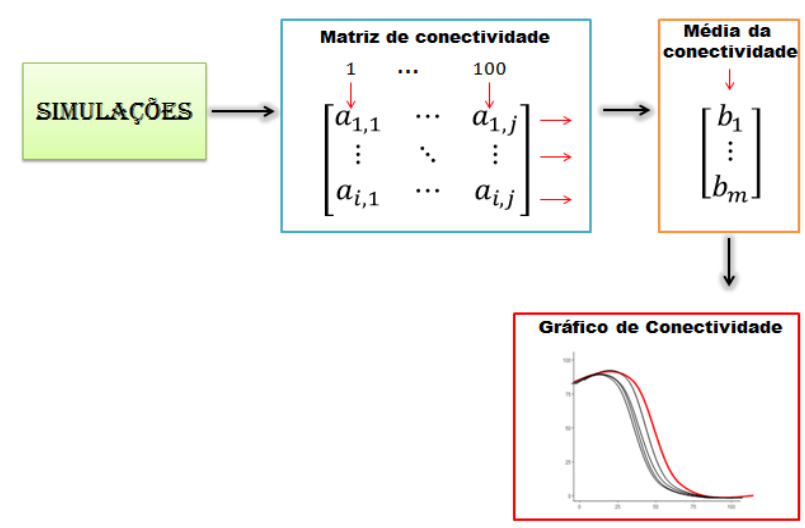

Figura 3.9 Representação do método de análise de conectividade. 


\section{APRESENTAÇÃO DOS RESULTADOS}

\subsection{Resultados}

Nesta seção, é evidenciado o desenvolvimento do algoritmo SIMDISPAT, aplicado a diferentes modelos complexos, com o objetivo de capturar certas características da imagem de treinamento, seja em 2D ou 3D. Considerando os parâmetros da seção anterior, as diversas simulações foram obtidas para cada banco de dados.

\subsection{Modelo de Canal Binário Bidimensional}

Usando os parâmetros da Tabela 3.9, foram calculadas cem realizações para o $\boldsymbol{B D} 1$. Das cem simulações, três realizações foram selecionadas aleatoriamente para cada distância proposta. A partir dos resultados da simulação condicional apresentados na Figura 4.1 observa-se que a distância de Lorentz $(\boldsymbol{D} \boldsymbol{L})$ e a distância Euclidiana $(\boldsymbol{D E})$ reproduzem os resultados com maior precisão, pois uma grande parte dos canais de $\boldsymbol{T I}$ são reproduzidos e devidamente conectados. A distância Manhattan $(\boldsymbol{D} \boldsymbol{M})$, também reproduziu algumas características da $\boldsymbol{T I}$, não obstante a união entre os canais é menor. Finalmente, a distância cosseno $(\boldsymbol{D C})$ foi a pior distância para simular canais complexos, dado que não reproduziu nenhuma das características da $\boldsymbol{T I}$.

Comparando a Tabela 3.1 com as Tabelas 4.1,4.2 e 4.3 evidencia-se uma proximidade entre os resultados das imagens simuladas e a $\boldsymbol{T I}$. Pela observação da Tabela 4.4, onde apresentam-se as estatísticas descritivas da $(\boldsymbol{D} \boldsymbol{C})$, as diferenças ficam evidentes, ou seja, o número de sucessos e a proporção não são próximos aos resultados mostrados na Tabela 3.1. 

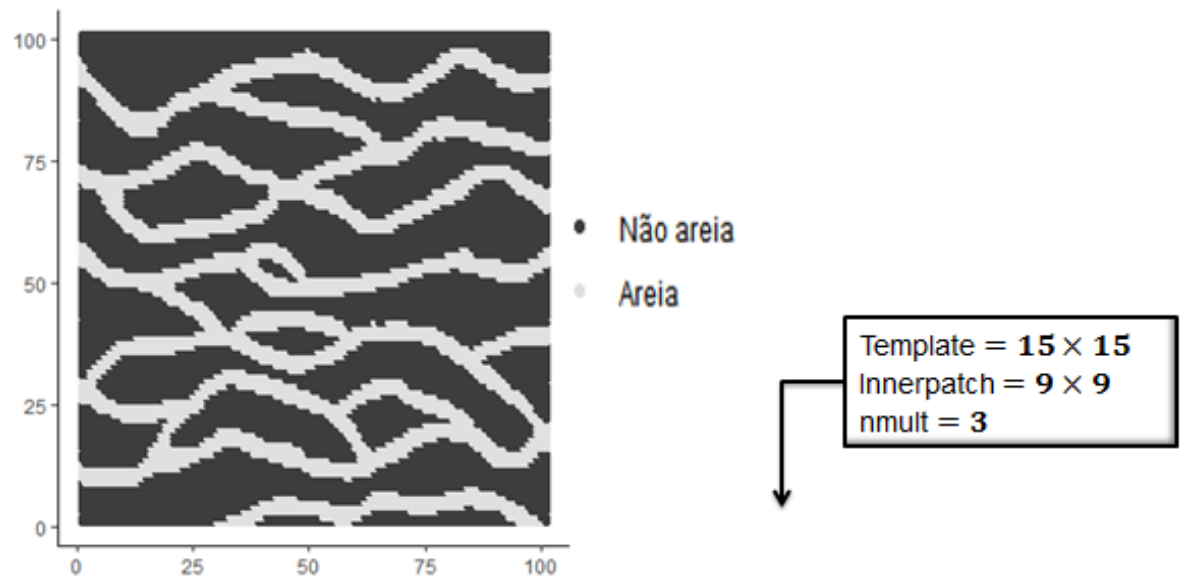

(a)
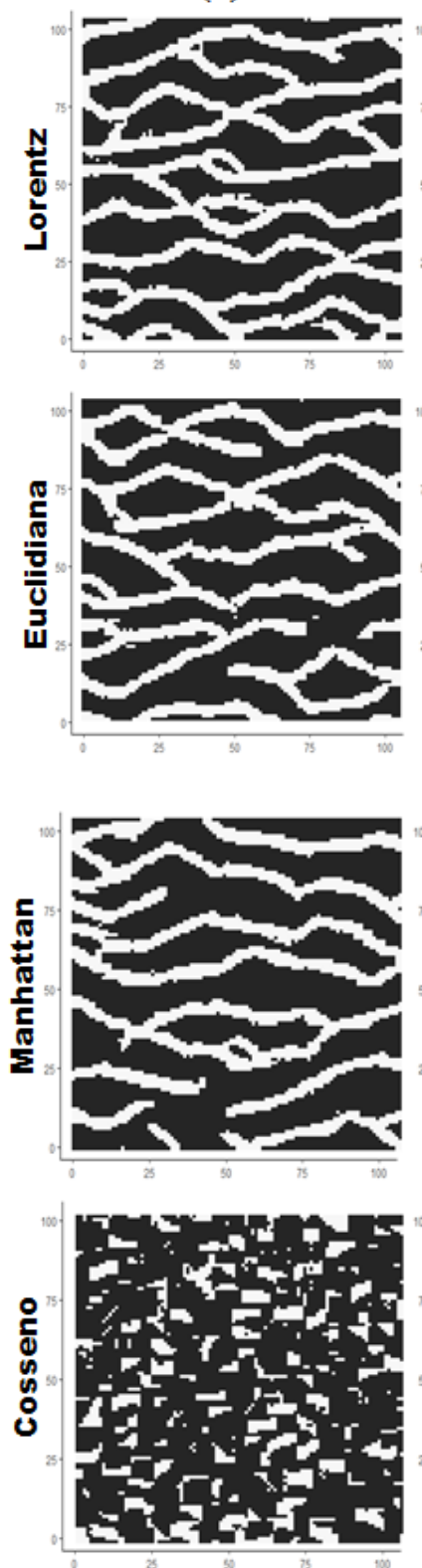

(b)
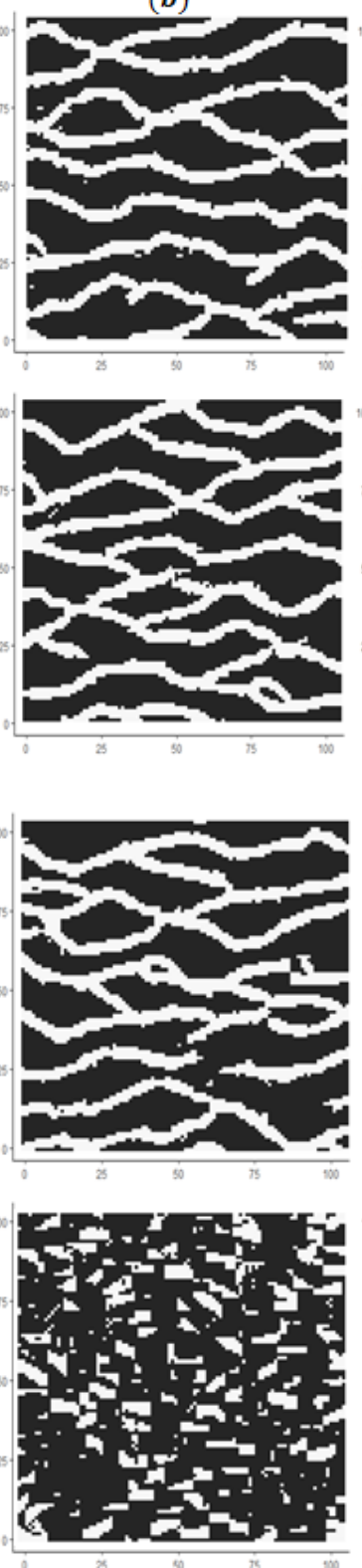

(c)
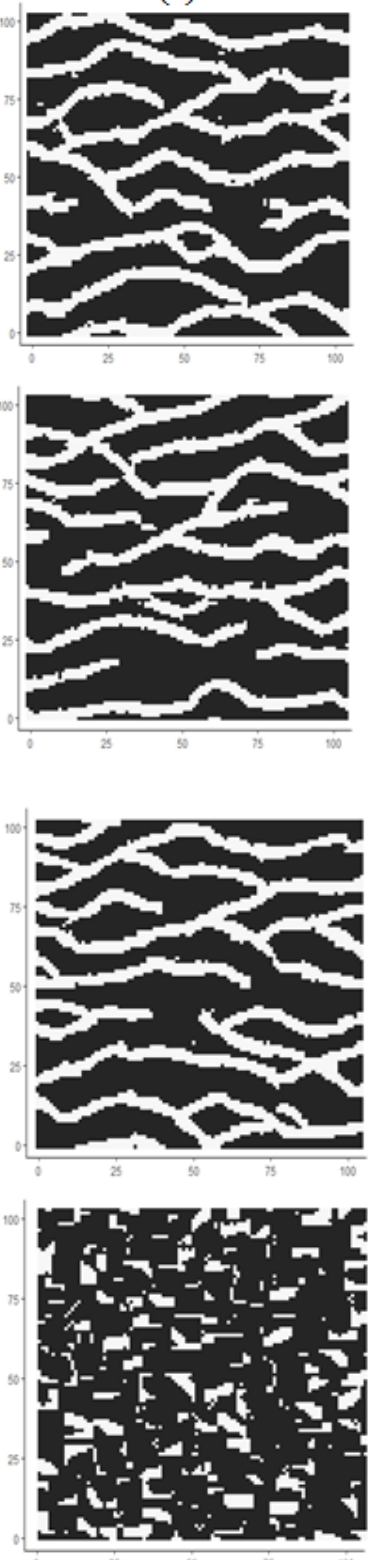

Figura 4.1 Resultados das simulações obtidas pelo algoritmo SIMDISPAT, para cada uma das quatro distâncias propostas, aplicado ao BD1 (a), (b) e (c) são três realizações tomadas ao acaso. 
Tabela 4.1 Proporção do tipo de litologia para as simulações selecionadas DL.

\begin{tabular}{ccccc}
\hline \multirow{2}{*}{ LITOLOGIA } & & \multicolumn{3}{c}{ SIMULAÇÃO } \\
\cline { 3 - 5 } & & DL38 $-(\boldsymbol{a})$ & DL5 $-(\boldsymbol{b})$ & DL37 - (c) \\
\hline Areia & Sucessos & 3587 & 3366 & 3302 \\
& Proporção & 0.351 & 0.329 & 0.323 \\
\hline \multirow{2}{*}{ Não Areia } & Sucessos & 6614 & 6835 & 6899 \\
& Proporção & 0.648 & 0.670 & 0.670 \\
\hline
\end{tabular}

Tabela 4.2 Proporção do tipo de litologia para as simulações selecionadas DE.

\begin{tabular}{ccccc}
\hline \multirow{2}{*}{ LITOLOGIA } & & \multicolumn{3}{c}{ SIMULAÇÃO } \\
\cline { 3 - 5 } & & DE5 - (a) & DE33 - (b) & DE55 - (c) \\
\hline Areia & Sucessos & 3318 & 3288 & 3190 \\
& Proporção & 0.325 & 0.322 & 0.312 \\
\hline \multirow{2}{*}{ Não Areia } & Sucessos & 6883 & 6913 & 7011 \\
& Proporção & 0.674 & 0.677 & 0.687 \\
\hline
\end{tabular}

Tabela 4.3 Proporção do tipo de litologia para as simulações selecionadas DM.

\begin{tabular}{ccccc}
\hline \multirow{2}{*}{ LITOLOGIA } & & \multicolumn{3}{c}{ SIMULAÇÃO } \\
\cline { 3 - 5 } & & DM17 - (a) & DM53 - (b) & DM1 - (c) \\
\hline Areia & Sucessos & 3080 & 3151 & 3364 \\
& Proporção & 0.301 & 0.308 & 0.329 \\
\hline \multirow{2}{*}{ Não Areia } & Sucessos & 7121 & 7050 & 6837 \\
& Proporção & 0.698 & 0.691 & 0.671 \\
\hline
\end{tabular}

Tabela 4.4 Proporção do tipo de litologia para as simulações selecionadas DC.

\begin{tabular}{ccccc}
\hline \multirow{2}{*}{ LITOLOGIA } & & \multicolumn{3}{c}{ SIMULAÇÃO } \\
\cline { 3 - 5 } & & $\mathbf{D C 1 3}-(\boldsymbol{a})$ & $\mathbf{D C 4 5}-(\boldsymbol{b})$ & $\mathbf{D C 1 9}-(\boldsymbol{c})$ \\
\hline Areia & Sucessos & 2032 & 2153 & 2063 \\
& Proporção & 0.199 & 0.211 & 0.202 \\
\hline \multirow{2}{*}{ Não Areia } & Sucessos & 8169 & 8048 & 8138 \\
& Proporção & 0.800 & 0.788 & 0.797 \\
\hline
\end{tabular}


A média de todas as imagens simuladas, conhecida como E-type, foi calculada para verificar a qualidade do condicionamento. Ilustra-se na Figura 4.2 o E-type das cem simulações para cada uma das distâncias. Os resultados mostram menor incerteza próximo aos pontos dos dados de condicionamento das Figuras $(4.2 a, 4.2 b$ e $4.2 c)$, porém a continuidade dos canais é mais perceptível no E-type de Lorentz. Os E-type mostrados nas Figuras $4.2 b$ e $4.2 c$ também apresentam uma boa estrutura nos canais, pois se aproximam do comportamento estatístico da TI. Não é possível reconhecer nenhuma estrutura de canal Figura $4.2 d$, portanto considera-se a incerteza maior.
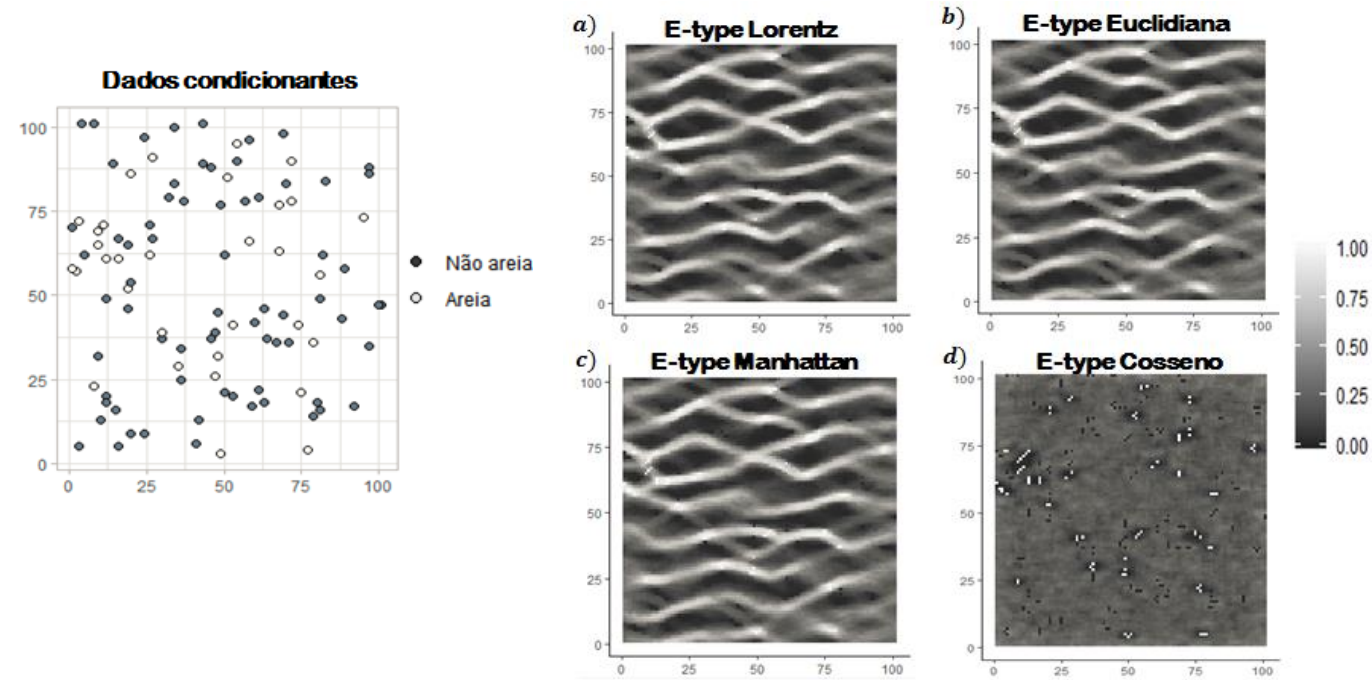

Figura 4.2 Os dados de condicionamento para o modelo do canal binário (à esquerda). O E-type das cem simulações geradas com o algoritmo SIMDISPAT, para cada uma das quatro distâncias (à direita).

\subsection{MODELO CATEGÓRICO DE QUATRO CATEGORIAS EM 2D}

Como nas simulações do modelo de canal binário, três realizações de cada uma das distâncias foram tomadas aleatoriamente. A simulação foi realizada usando os parâmetros apresentados na Tabela 3.9. Apresentam-se na Figura 4.3 os resultados das três realizações selecionadas para cada distância. Observa-se que $\boldsymbol{D} \boldsymbol{L}$ e $\boldsymbol{D} \boldsymbol{M}$ são os que apresentam os melhores resultados, dado que, os canais têm boa continuidade e a sequência da união entre as fácies é reproduzida adequadamente. Já na $\boldsymbol{D E}$, pode-se observar que 
existem várias amostras que não foram adequadamente condicionadas, embora esta distância consiga capturar algumas das características da TI. Por último nos resultados da $\boldsymbol{D C}$, fica evidente que as realizações selecionadas não reproduzem nenhum dos padrões encontrados na $\boldsymbol{T I}$.

Comparando as estatísticas básicas da $\boldsymbol{T I}$, apresentadas na Tabela 3.2, com as simuladas, observa-se que as Tabelas 4.5 e 4.7, que representam os resultados das simulações da $\boldsymbol{D} \boldsymbol{L}$ e da $\boldsymbol{D M}$, respectivamente, são as mais próximas, tanto para o número de sucessos, como para as proporções. $\mathrm{Na}$ Tabela 4.6, que contêm as estatísticas da $\boldsymbol{D E}$, os resultados são disparesdaqueles contidos na Tabela 3.2. Finalmente, observando-se a Tabela 4.8, é evidente que os resultados obtidos não se aproximam àqueles mostrados na Tabela 3.2.

Mostra-se na Figura 4.4 as quatro imagens de E-type, calculadas para as simulações utilizando cada uma das distâncias. Visualmente os resultados das Figuras $4.4 a$ e $4.4 c$ mostram uma reprodução eficaz das estruturas de canal em torno das localizações dos dados de condicionamento. Por outro lado, na Figura $4.4 b$ é possível inferir que a continuidade dos canais foi razoavelmente reproduzida. Pela interpretação do E-type da $\boldsymbol{D E}$, pode-se observar que são criadas outras estruturas, diferentes daquelas da TI. Por fim, na Figura $4.4 d$ é evidente que os padrões não são reproduzidos no E-type calculado para a $\boldsymbol{D C}$. 

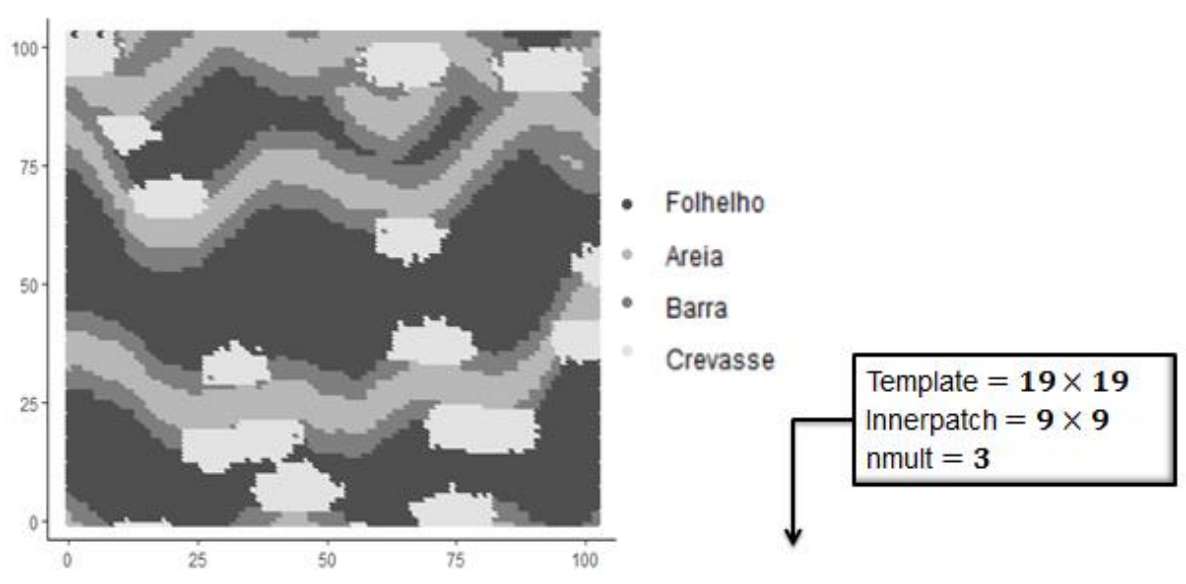

(a)
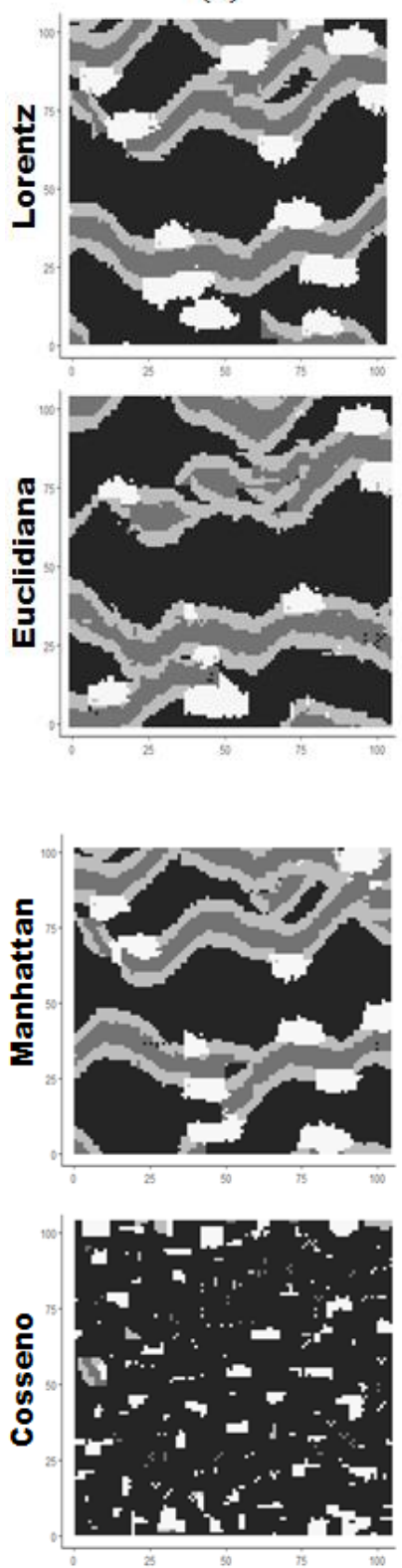

(b)
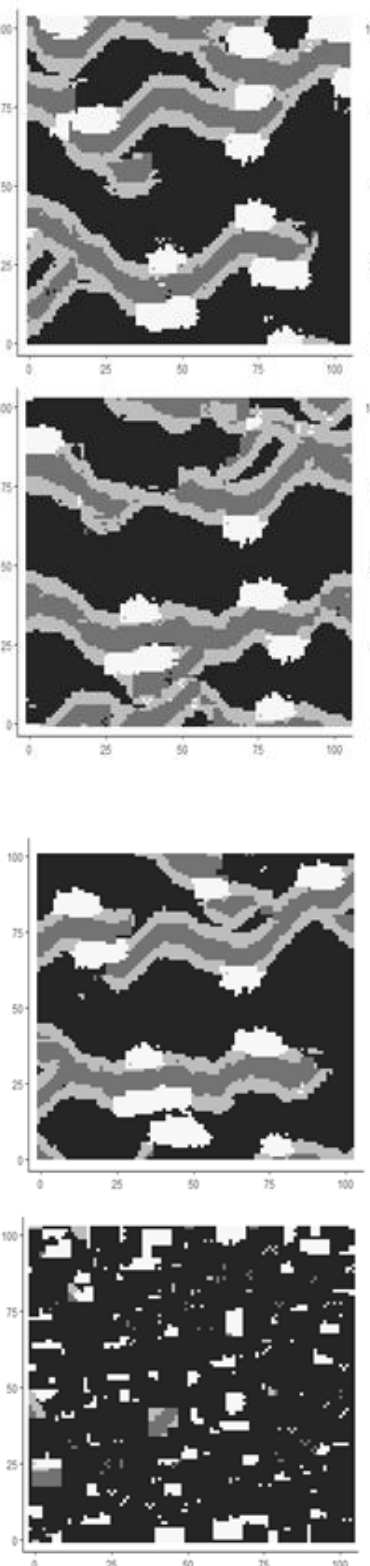

(c)
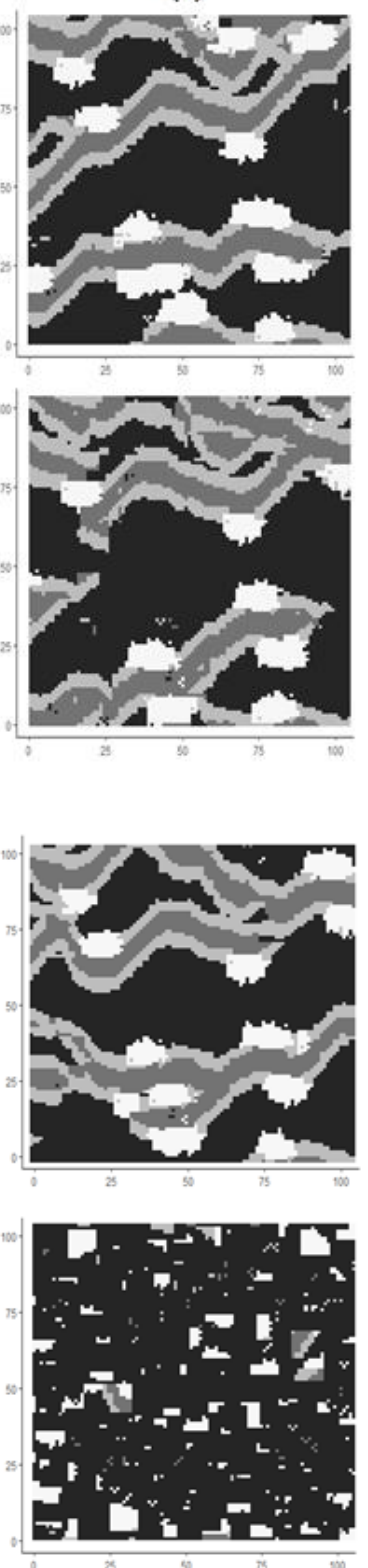

Figura 4.3 Resultados das simulações obtidas pelo algoritmo SIMDISPAT, para cada uma das quatro distâncias propostas, aplicado ao BD2. 
Tabela 4.5 Proporção do tipo de litologia para as simulações selecionadas DL.

\begin{tabular}{llcccc}
\hline \multirow{2}{*}{ SIMULAÇÃo } & & \multicolumn{4}{c}{ CATEGORIAS } \\
\cline { 3 - 6 } & & Crevasse & Folhelho & Areia & Barra \\
\hline DL30 $-(\boldsymbol{a})$ & Sucessos & 1423 & 4981 & 2002 & 1795 \\
& Proporção & 0.139 & 0.488 & 0.196 & 0.175 \\
\hline DL91 - (b) & Sucessos & 1262 & 5013 & 2013 & 1913 \\
& Proporção & 0.123 & 0.491 & 0.197 & 0.187 \\
\hline DL54 - $(\boldsymbol{c})$ & Sucessos & 1334 & 4818 & 1972 & 2077 \\
& Proporção & 0.130 & 0.472 & 0.193 & 0.203 \\
\hline
\end{tabular}

Tabela 4.6 Proporção do tipo de litologia para as simulações selecionadas DE.

\begin{tabular}{llcccc}
\hline SIMULAÇÃO & & \multicolumn{4}{c}{ CATEGORIAS } \\
\cline { 3 - 6 } & & Crevasse & Folhelho & Areia & Barra \\
\hline \multirow{2}{*}{ DE55 $-(\boldsymbol{a})$} & Sucessos & 1027 & 5004 & 1891 & 2279 \\
& Proporção & 0.100 & 0.490 & 0.185 & 0.223 \\
\hline \multirow{2}{*}{ DE73 - (b) } & Sucessos & 875 & 5083 & 1973 & 2270 \\
\cline { 3 - 6 } & Proporção & 0.085 & 0.498 & 0.193 & 0.222 \\
\hline \multirow{2}{*}{ DE92 - $(\boldsymbol{c})$} & Sucessos & 593 & 5069 & 2073 & 2466 \\
& Proporção & 0.058 & 0.496 & 0.203 & 0.241 \\
\hline
\end{tabular}

Tabela 4.7 Proporção do tipo de litologia para as simulações selecionadas DM.

\begin{tabular}{llcccc}
\hline \multirow{2}{*}{ SIMULAÇÃo } & & \multicolumn{4}{c}{ CATEGORIAS } \\
\cline { 3 - 6 } & & Crevasse & Folhelho & Areia & Barra \\
\hline \multirow{2}{*}{ DM46 - $(\boldsymbol{a})$} & Proporção & 0.093 & 0.491 & 0.191 & 0.210 \\
\hline \multirow{2}{*}{$\mathbf{D}$} & Sucessos & 1180 & 5578 & 1699 & 1745 \\
\hline \multirow{2}{*}{$\mathbf{D}-(\boldsymbol{b})$} & Proporção & 0.115 & 0.546 & 0.166 & 0.171 \\
\cline { 3 - 6 }$-(\boldsymbol{c})$ & Sucessos & 1130 & 4953 & 2011 & 2107 \\
& Proporção & 0.110 & 0.485 & 0.197 & 0.206 \\
\hline
\end{tabular}


Tabela 4.8 Proporção do tipo de litologia para as simulações selecionadas DC.

\begin{tabular}{llcccc}
\hline SIMULAÇÃO & & \multicolumn{4}{c}{ CATEGORIAS } \\
\cline { 3 - 6 } & & Crevasse & Folhelho & Areia & Barra \\
\hline \multirow{2}{*}{$\mathbf{D C 4 0 ~}-(\boldsymbol{a})$} & Sucessos & 1122 & 8775 & 157 & 147 \\
& Proporção & 0.109 & 0.860 & 0.015 & 0.014 \\
\hline \multirow{2}{*}{$\mathbf{D C 9 2}-(\boldsymbol{b})$} & Sucessos & 981 & 8993 & 90 & 137 \\
\cline { 3 - 6 } & Proporção & 0.096 & 0.881 & 0.008 & 0.013 \\
\hline \multirow{2}{*}{$\mathbf{D C 1 1}-(\boldsymbol{c})$} & Sucessos & 903 & 9060 & 78 & 160 \\
& Proporção & 0.088 & 0.888 & 0.007 & 0.015 \\
\hline
\end{tabular}

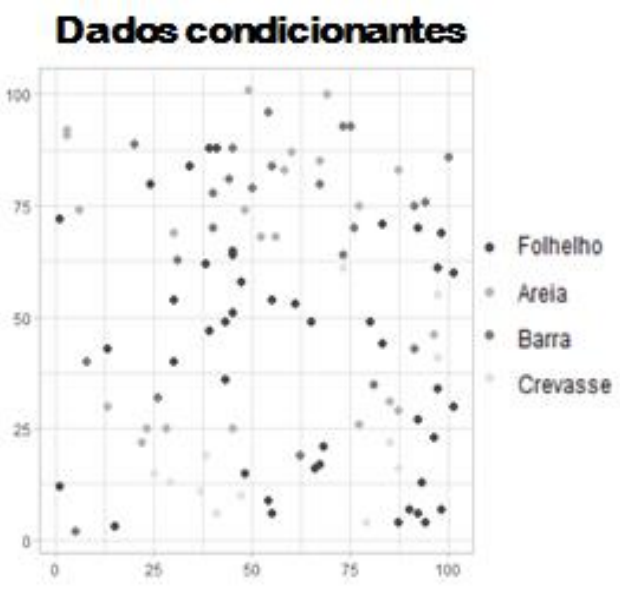

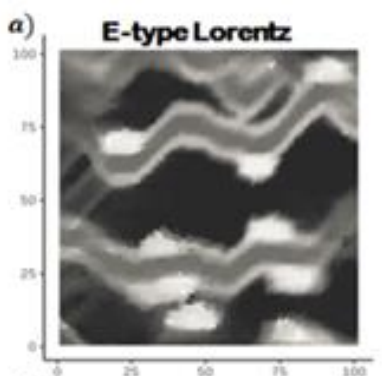
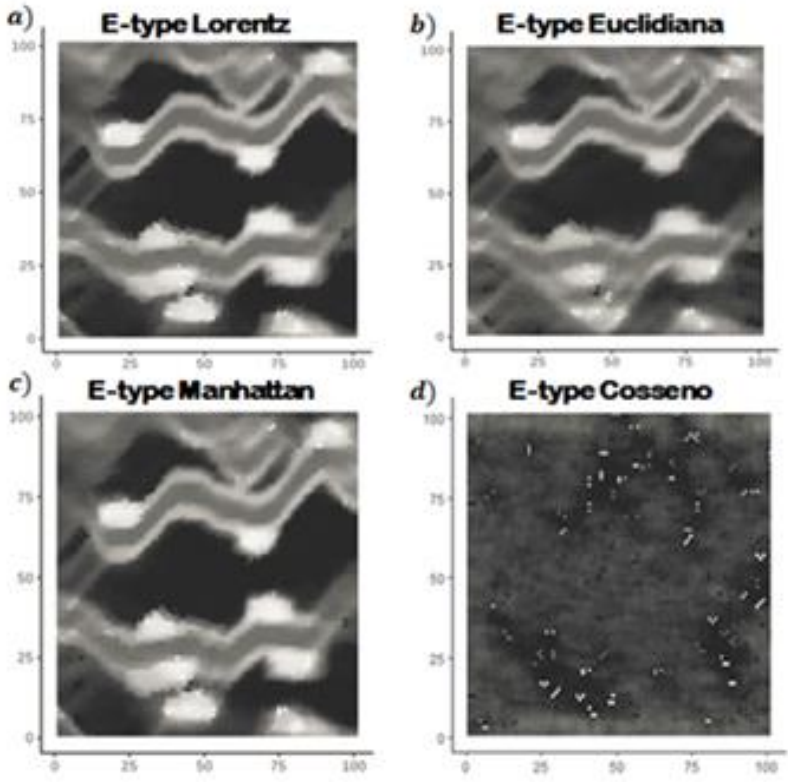

Figura 4.4 Os dados de condicionamento para o modelo de quatro categorias (à esquerda). O E-type das 100 simulações geradas com o algoritmo SIMDISPAT, para cada uma das 4 distâncias selecionadas (à direita).

\subsection{Modelo Contínuo Bidimensional}

Foram geradas cem realizações condicionais, das quais três foram escolhidas aleatoriamente para cada uma das distâncias, conforme apresentado na Figura 4.5. Nas simulações do modelo contínuo do muro de pedra, foram utilizados os parâmetros apresentados na Tabela 3.9. Mostra-se 
na Figura 4.5 que as distâncias $\boldsymbol{D} \boldsymbol{L}, \boldsymbol{D} \boldsymbol{E}$ e $\boldsymbol{D} \boldsymbol{M}$ apresentam são consistentes ao capturar a textura do muro de pedra, pode-se observar que algumas descontinuidades sutis foram criadas. Finalmente, os resultados das simulações para a $\boldsymbol{D C}$ evidenciam que nenhuma das texturas da imagem de treinamento é capturada.

Algumas estatísticas descritivas das $\boldsymbol{D L}, \boldsymbol{D E}$ e $\boldsymbol{D} \boldsymbol{M}$ (mínimo, máximo, quartís e média) são apresentadas nas Tabelas 4.9, 4.10 e 4.11, pelos resultados pode-se inferir que as diferenças no valor das médias e quartis são mínimas em relação aos resultados apresentados na Tabela. 3.3. Observandose a Tabela 4.12 percebe-se que as estatisticas descritivas analisadas não foram reproduzidas quando comparadas àquelas da Tabela 3.3.

Apresenta-se na Figura 4.6 os resultados do E-type calculados a partir das 100 realizações das simulações para cada distância. Observando-se as Figuras $4.6 a, 4.6 b$ e $4.6 c$ é evidente que existem pequenas variações entre as realizações, porém são semelhantes, podendo-se afirmar que as características do muro de pedra foram reproduzidas adequadamente. Além disso, pode-se observar que as realizações se ajustam ao mapa de localização dos 400 dados de condicionamento. Finalmente, observa-se que o E-type apresentado na Figura $4.6 d$ não reproduz nenhuma das características da $\boldsymbol{T I}$. 


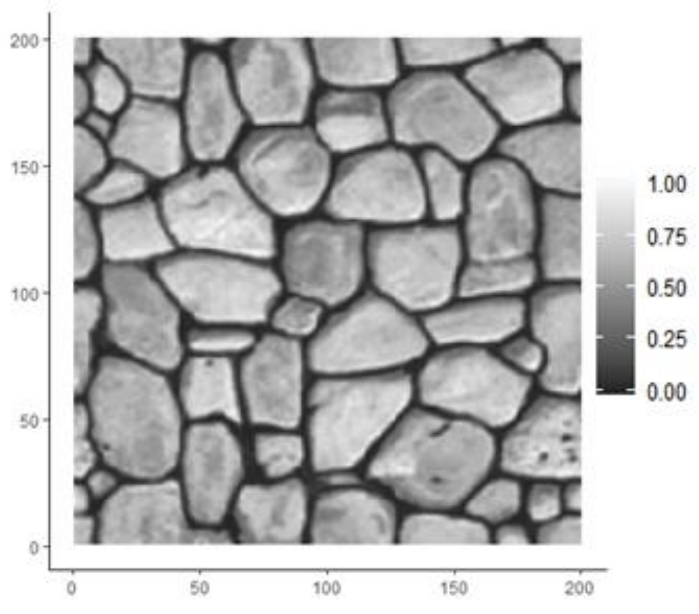

(a)
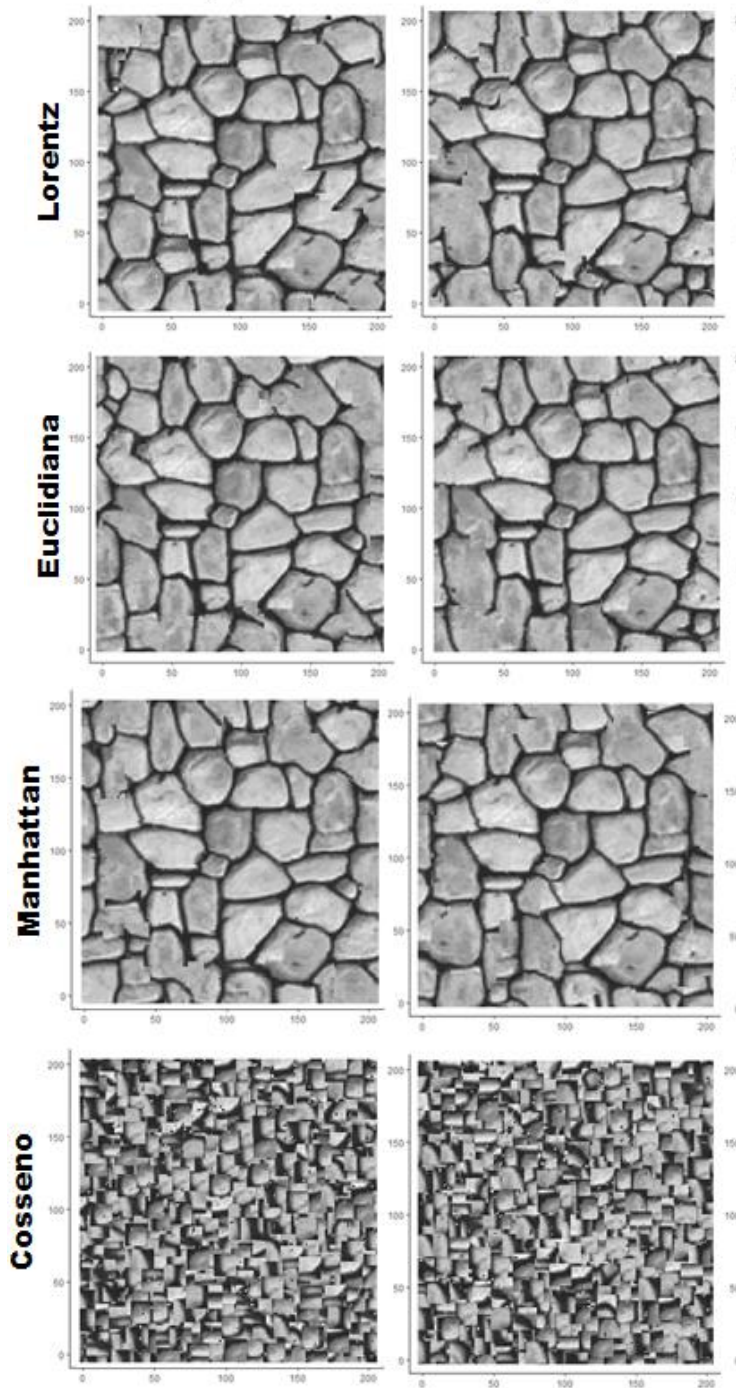

(b)
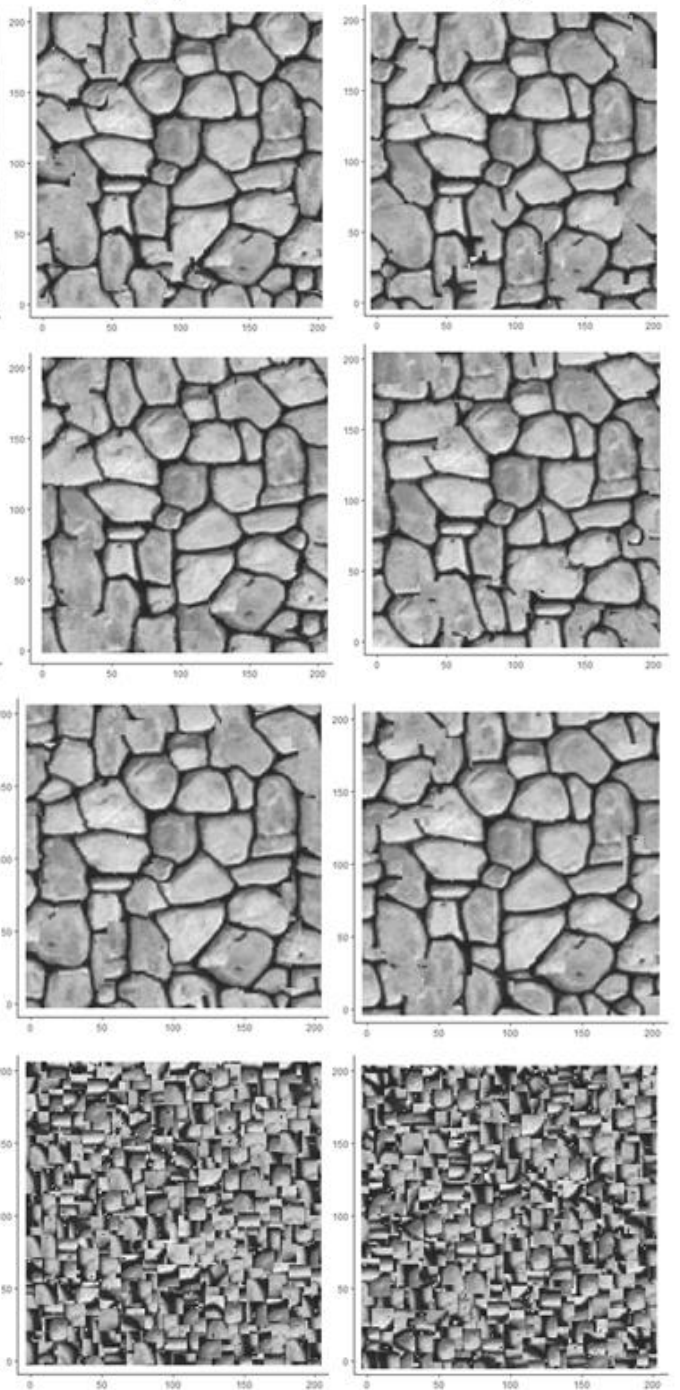

Template $=\mathbf{2 1} \times \mathbf{2 1}$

Innerpatch $=\mathbf{1 5} \times \mathbf{1 5}$

nmult $=3$

$(c)$
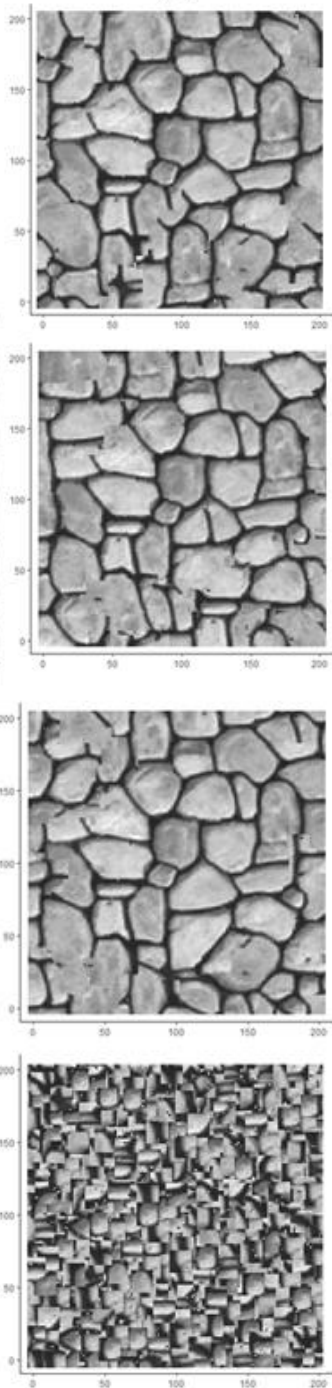

Figura 4.5 Resultados das simulações obtidas pelo algoritmo SIMDISPAT, para cada uma das quatro distâncias propostas, aplicado ao BD3. 
Tabela 4.9 Estatísticas descritivas para as realizações selecionadas da Figura 4.5, com respeito a $\mathbf{D L}$.

\begin{tabular}{lcccccc}
\hline SIMULAÇÃO & Mín & 1QQuartil & Mediana & Média & 3 Quartil & Máx \\
\hline DL1 $(\boldsymbol{a})$ & 0.000 & 0.356 & 0.588 & 0.514 & 0.690 & 1.000 \\
DL95(b) & 0.000 & 0.364 & 0.584 & 0.507 & 0.675 & 1.000 \\
DL51(c) & 0.000 & 0.349 & 0.580 & 0.504 & 0.678 & 1.000 \\
\hline
\end{tabular}

Tabela 4.10 Estatísticas descritivas para as realizações selecionadas da Figura 4.5, com respeito à $\mathbf{D E}$.

\begin{tabular}{lcccccc}
\hline SIMULAÇÃO & Mín & 1Quartil $^{\circ}$ & Mediana & Média & 3o Quartil & Máx \\
\hline DE60 $(\boldsymbol{a})$ & 0.000 & 0.360 & 0.580 & 0.506 & 0.670 & 1.000 \\
DE4(b) & 0.000 & 0.341 & 0.576 & 0.504 & 0.678 & 1.000 \\
DE15 $(\boldsymbol{c})$ & 0.000 & 0.376 & 0.577 & 0.509 & 0.671 & 1.000 \\
\hline
\end{tabular}

Tabela 4.11 Estatísticas descritivas para as realizações selecionadas da Figura 4.5, com respeito a DM.

\begin{tabular}{lcccccc}
\hline SIMULAÇÃO & Mín & 1Quartil & Mediana & Média & 3 Quartil & Máx \\
\hline DM2(a) & 0.000 & 0.364 & 0.584 & 0.510 & 0.674 & 1.000 \\
DM60(b) & 0.000 & 0.360 & 0.572 & 0.505 & 0.670 & 1.000 \\
DM96(c) & 0.000 & 0.352 & 0.576 & 0.504 & 0.671 & 1.000 \\
\hline
\end{tabular}

Tabela 4.12 Estatísticas descritivas para as realizações selecionadas da Figura 4.5, com respeito a DC.

\begin{tabular}{lcccccc}
\hline SIMULAÇÃO & Mín & 1QQuartil & Mediana & Média & 3Quartil & Máx \\
\hline DC23(a) & 0.000 & 0.105 & 0.407 & 0.392 & 0.639 & 1.000 \\
DC100(b) & 0.000 & 0.113 & 0.431 & 0.402 & 0.647 & 1.000 \\
DC41(c) & 0.000 & 0.086 & 0.407 & 0.484 & 0.639 & 1.000 \\
\hline
\end{tabular}



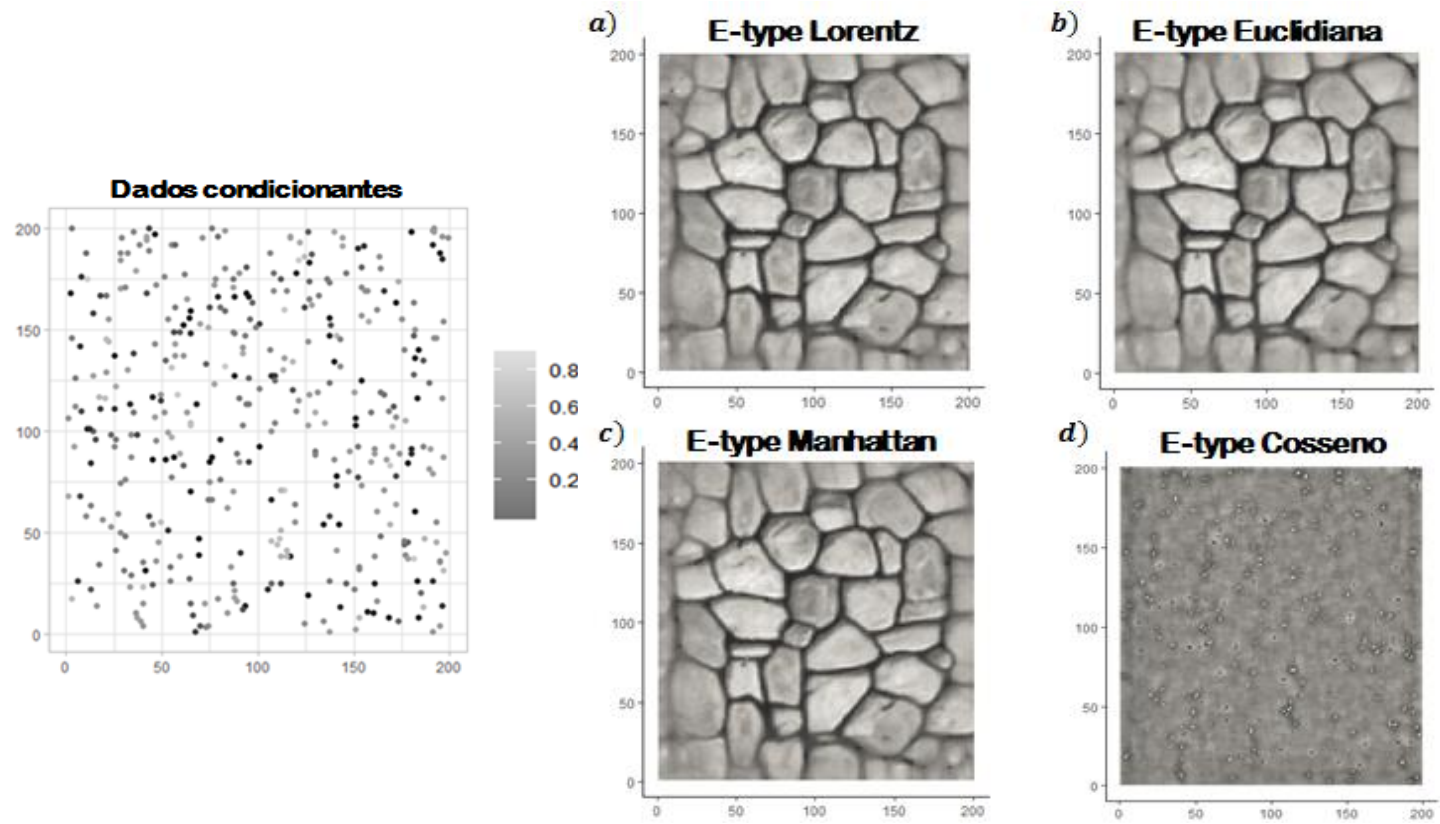

Figura 4.6 Os dados de condicionamento para o modelo contínuo bidimensional (à esquerda). É observada o E-type das cem simulações geradas com o algoritmo SIMDISPAT, para cada uma das 4 distâncias selecionadas (à direita).

\subsection{Modelo Binário Tridimensional}

Para verificar a eficiência do algoritmo SIMDISPAT, foi aplicado em um banco de dados tridimensional, o qual é um modelo binário de folhelho e areia. Para as simulações, foram utilizados os parâmetros apresentados na Tabela 3.9. Como descrito para as bases de dados apresentadas anteriormente para modelo 3D foram escolhidas ao acaso três realizações, para cada distância, com o objetivo de fazer as devidas comparações. Deve-se destacar que como a $\boldsymbol{D C}$ não reproduziu nenhum padrão das $\boldsymbol{T I}$ anteriores ela não foi aplicada aqui. Pode-se observar na Figura 4.7, que das três distâncias utilizadas a $\boldsymbol{D L}$ e a $\boldsymbol{D} \boldsymbol{M}$ reproduzem com eficiência os padrões encontrados na $\boldsymbol{T I}$. Além disso, pode-se dizer que o algoritmo SIMDISPAT reproduz adequadamente o modelo geológicos em 3D. Fazendo uma comparação das proporções obtidas nas simulações (Tabelas 4.13, 4.14 e 4.15) com as das imagens de treinamento (Tabela 3.4) fica evidente que a $\boldsymbol{D} \boldsymbol{L}$ e a $\boldsymbol{D} \boldsymbol{M}$ foram as que apresentaram os resultados mais próximos das proporções existentes na $\boldsymbol{T I}$. 

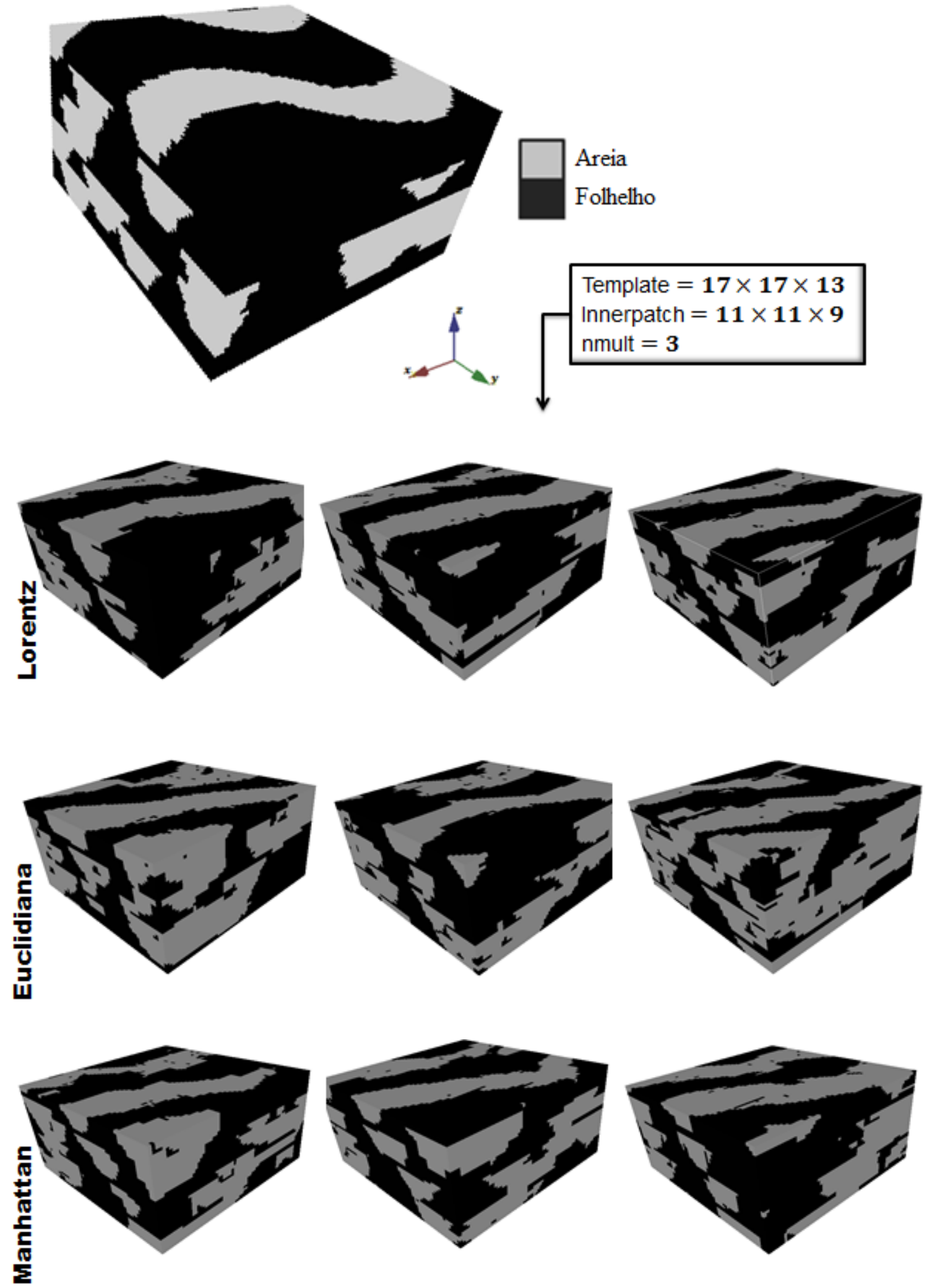

Figura 4.7 Resultados das simulações obtidas pelo algoritmo SIMDISPAT, para cada uma das três distâncias, aplicada ao BD4. 
Tabela 4.13 Proporção do tipo de litologia para as simulações selecionadas DL.

\begin{tabular}{ccccc}
\hline \multirow{2}{*}{ LITOLOGIA } & & \multicolumn{3}{c}{ SIMULAÇÃO } \\
\cline { 3 - 5 } & & DL36-(a) & DL22 - (b) & DL9 - (c) \\
\hline Areia & Sucessos & 76255 & 77265 & 77630 \\
& Proporção & 0.410 & 0.416 & 0.418 \\
\hline \multirow{2}{*}{ Folhelho } & Sucessos & 109424 & 108414 & 108049 \\
& Proporção & 0.589 & 0.583 & 0.581 \\
\hline
\end{tabular}

Tabela 4.14 Proporção do tipo de litologia para as simulações selecionadas DE

\begin{tabular}{ccccc}
\hline \multirow{2}{*}{ LITOLOGIA } & & \multicolumn{3}{c}{ SIMULAÇÃO } \\
\cline { 3 - 5 } & & DE32 $-(\boldsymbol{a})$ & DE81 $-(\boldsymbol{b})$ & DE92 $-(\boldsymbol{c})$ \\
\hline Areia & Sucessos & 82194 & 85479 & 87078 \\
& Proporção & 0.442 & 0460 & 0.468 \\
\hline \multirow{2}{*}{ Folhelho } & Sucessos & 103485 & 100200 & 98601 \\
& Proporção & 0.557 & 0.539 & 0.531 \\
\hline
\end{tabular}

Tabela 4.15 Proporção do tipo de litologia para as simulações selecionadas DM.

\begin{tabular}{ccccc}
\hline \multirow{2}{*}{ LITOLOGIA } & & \multicolumn{3}{c}{ SIMULAÇÃO } \\
\cline { 3 - 5 } & & DM11 $-(\boldsymbol{a})$ & DM50 - (b) & DM73 - (c) \\
\hline Areia & Sucessos & 73757 & 83973 & 68936 \\
& Proporção & 0.397 & 0.452 & 0.371 \\
\hline \multirow{2}{*}{ Folhelho } & Sucessos & 111922 & 101706 & 116743 \\
& Proporção & 0.602 & 0.547 & 0.628 \\
\hline
\end{tabular}


Para verificar a qualidade do condicionamento, o E-type das cem realizações foi calculado conforme pode ser observado na Figura 4.8. $\mathrm{Na}$ Figura $4.8 c$, verifica-se que as características dos canais apresentam melhor nitidez, com relação às Figuras $4.8 a$ e $4.8 b$.
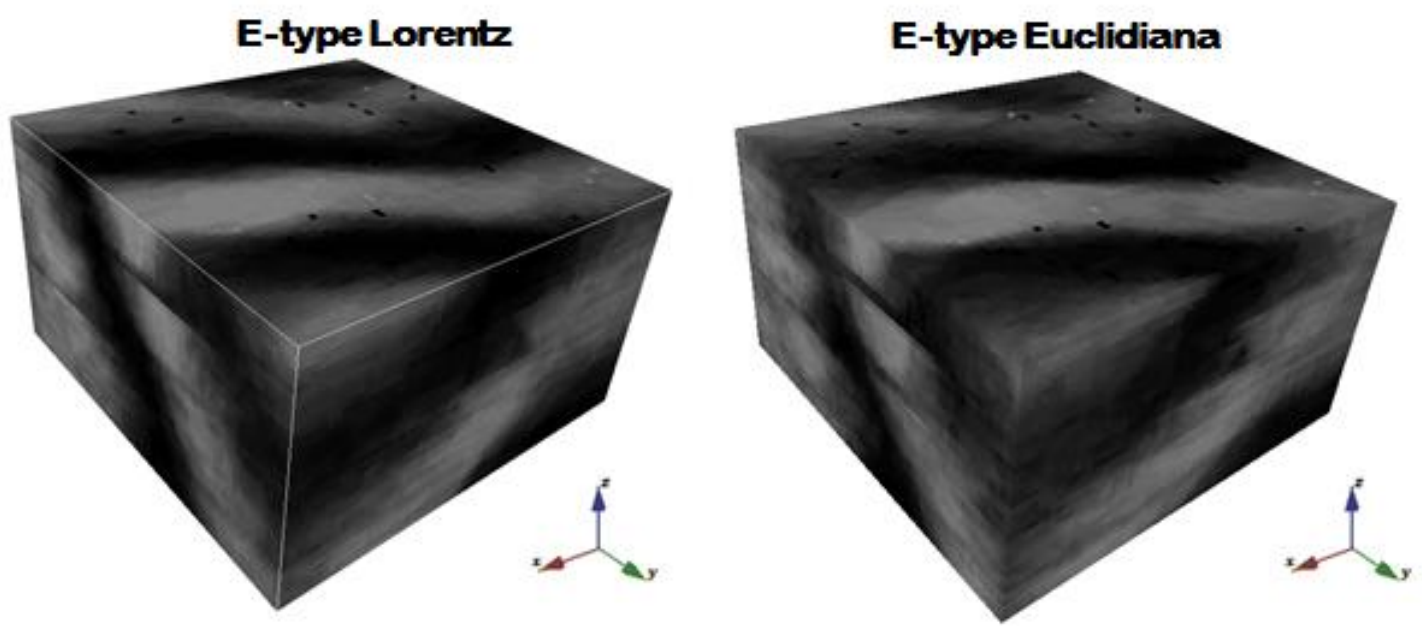

\section{E-type Manhattan}

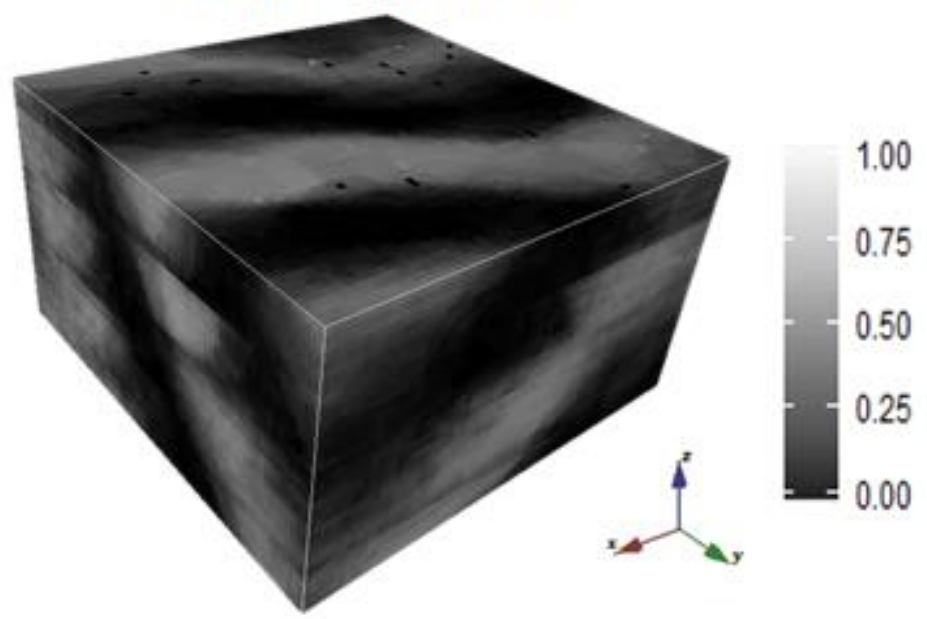

Figura 4.8 E-type das cem simulações geradas com o algoritmo SIMDISPAT, para cada uma das três distâncias selecionadas (BD4 ). 


\subsection{Análise de Conectividade}

Para verificar os resultados das simulações, é necessário verificar quantitativamente a reprodução das características complexas presentes nas realizações obtidas pelo algoritmo SIMDISPAT. Para cada uma das realizações, espera-se que a conectividade dos resultados seja elevada e, consequentemente, semelhante à imagem de treinamento.

A função CONNEC3D (PARDO-IGUZQUIZA \& DOWD, 2003) realiza a análise de conectividade para cada uma das realizações geradas. As Figuras 4.9, 4.10 e 4.11 ilustram o estudo de conectividade para cada uma das realizações obtidas no SIMDISPAT para, respectivamente o BD1, BD2 e BD4. Além disso, cada analise de conectividade foram calculadas em direção Azimute $=90^{\circ}$, dado que a conectividade de longo alcance dos canais estava na direção Leste-Oeste.

$\mathrm{Na}$ figura 4.9 observa-se que os resultados indicam que existem oscilações, além disso, é evidente que a conectividade das realizações obtidas com a $\boldsymbol{D} \boldsymbol{L}$ e com a $\boldsymbol{D E}$ são as que melhor se aproximam da conectividade da TI. Observa-se que a curva da $\boldsymbol{D} \boldsymbol{M}$ apresenta uma ligeira separação em relação àquelas da $\boldsymbol{D E}$ e da $\boldsymbol{D L}$, o qual a $\boldsymbol{D M}$ representa maior variabilidade. Isso pode ser devido ao fato de que a reprodução dos padrões é regular. Finalmente a $\boldsymbol{D C}$ não consegue reproduzir a conectividade dos canais das simulações, observe-se que os seus resultados de conectividade estão bem distantes dos da $\boldsymbol{T I}$. 


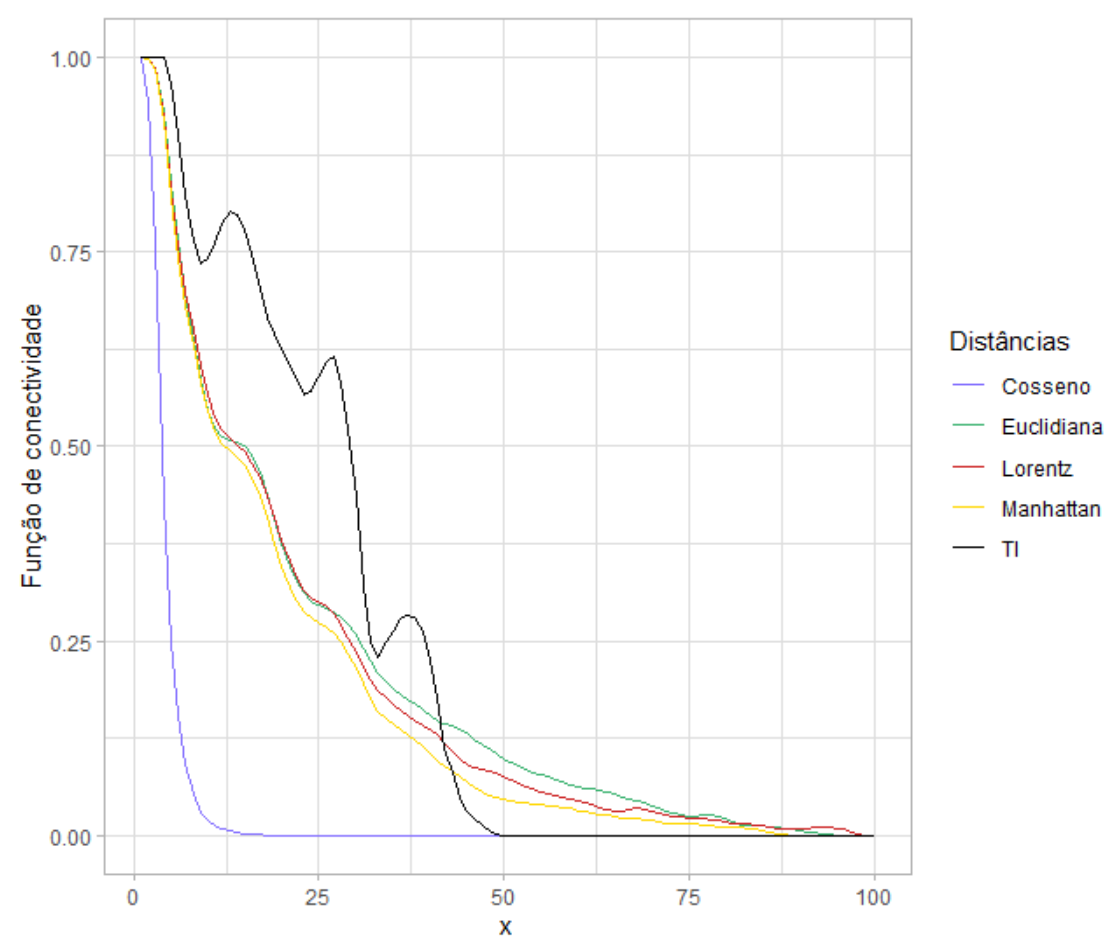

Figura 4.9 Função de conectividade para as realizações geradas por SIMDISPAT. As curvas coloridas tracejadas representam o resultado obtido para cada uma das cem simulações calculadas com cada uma das distâncias e a curva sólida preta é a conectividade obtida na $\boldsymbol{T I}$.

$\mathrm{Na}$ Figura 4.10 se mostra os resultados do analises de conectividade para o BD2 (TI com quatro categorias), indicam que nessa direção a $D L$ e a $\boldsymbol{D} \boldsymbol{M}$ apresentam boa semelhança entre as realizaçoes e a $\boldsymbol{T I}$, dado que a maioria das realizações capturou uma grande parte dos padrões. Por outro lado, a $\boldsymbol{D E}$ produz uma conectividade um pouco mais afastada daquela da imagem de treinamento, criando variabilidade e apresentando diferentes comportamentos. Finalmente, mostra-se na Figura 4.10 que a conectividade da $\boldsymbol{D C}$, está bastante afastada da $\boldsymbol{T I}$, o que significa que não conseguiu reproduzir nenhum dos padrões obtidos na $\boldsymbol{T I}$. 


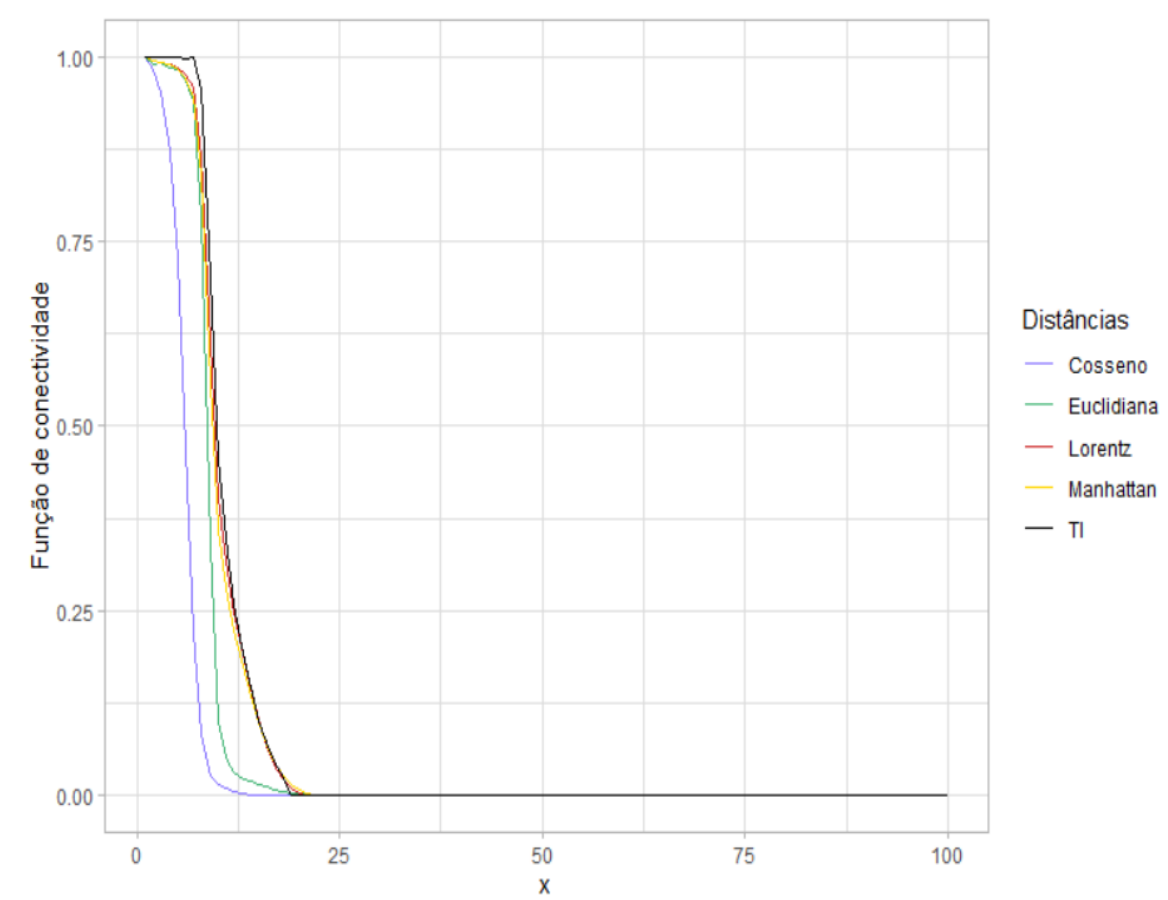

Figura 4.10 A função de conectividade calculada para as realizações geradas da figura 4.3. As curvas coloridas representam as realizações de cada uma das distâncias e a TI é mostrado de cor preto.

Para a Figura 4.11 observa-se que a $\boldsymbol{D} \boldsymbol{L}$ e a $\boldsymbol{D} \boldsymbol{M}$ tem maior similaridade com a TI e, por conseguinte, observa-se a melhor reprodução dos padrões. Em relação à $\boldsymbol{D E}$, observa-se que as realizações são mais distantes da $\boldsymbol{T I}$ aumentando a variabilidade e criando diferentes padrões.

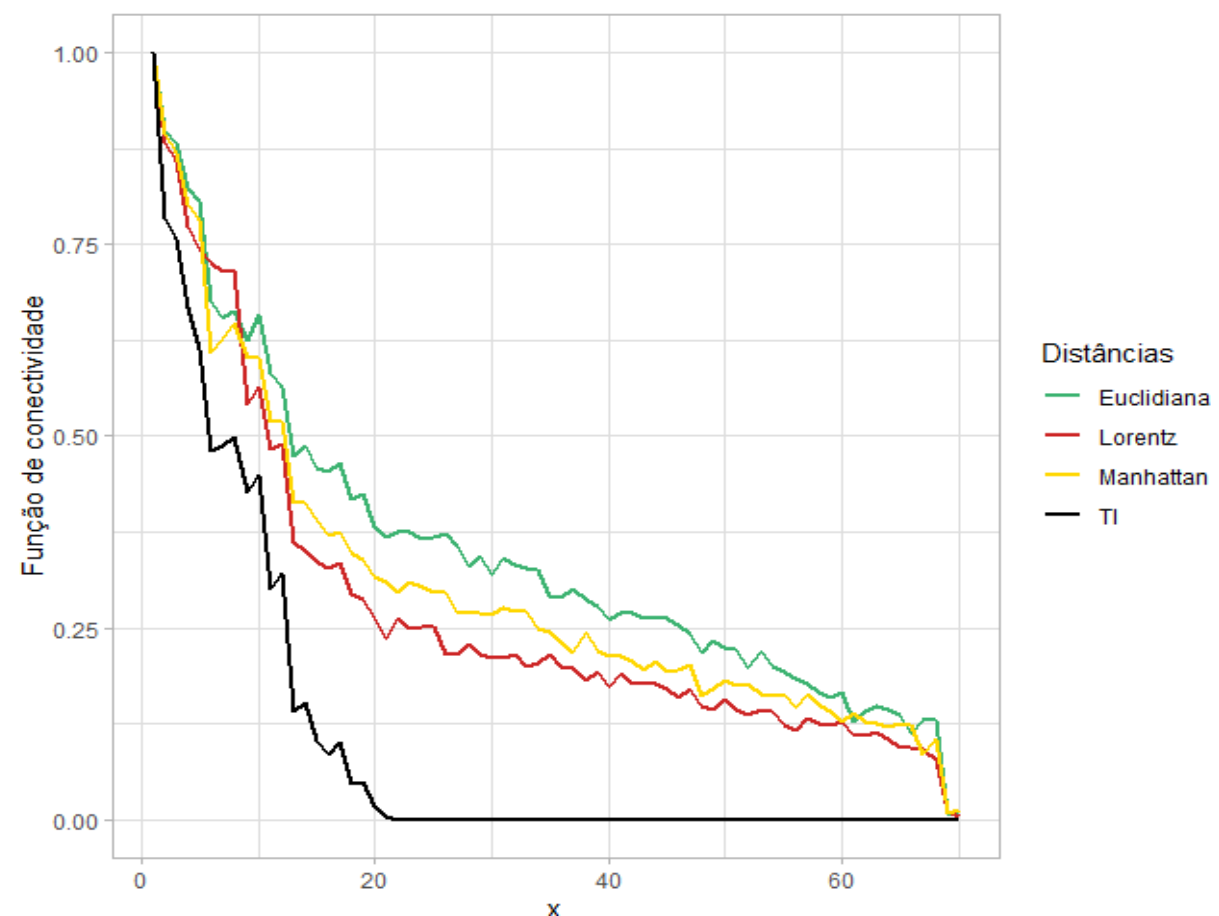

Figura 4.11 A função de conectividade calculada para as realizações geradas. 


\subsection{Análise por Escalonamento Multidimensional (MDS)}

Para comparar ainda mais os modelos obtidos a técnica de análise MDS foi realizada, pois se pode fazer a inspeção visual para verificar a variabilidade das simulações geradas. Ressalta-se que o MDS foi calculado com os valores obtidos nas simulações pelo o algoritmo de SIMDISPAT, onde a matriz de distância é calculada pixel a pixel entre as imagens estocásticas.

Observa-se na Figura $4.12 a$ que existe maior concentração de pontos em torno do ponto da $\boldsymbol{T I}$ do $\boldsymbol{D B} \mathbf{1}$ nos resultados utilizando a $\boldsymbol{D} \boldsymbol{M}$ e a $\boldsymbol{D} \boldsymbol{L}$. A nuvem de pontos obtidos para a $\boldsymbol{D E}$ está mais dispersa em relação ao ponto da TI. Por último, observa-se pelos resultados das simulações utilizando a $\boldsymbol{D C}$ que esta distância não reproduz a continuidade dos canais, uma vez que todos os pontos estão distantes da $\boldsymbol{T I}$. Na Figura $4.12 b$ observa-se o ponto médio de cada uma das nuvens de pontos. Pode-se observar que em média as distâncias que mais se aproximam da $\boldsymbol{T I}$ são a $\boldsymbol{D} \boldsymbol{M}$ e a $\boldsymbol{D L}$. 


\section{MDS}

a)

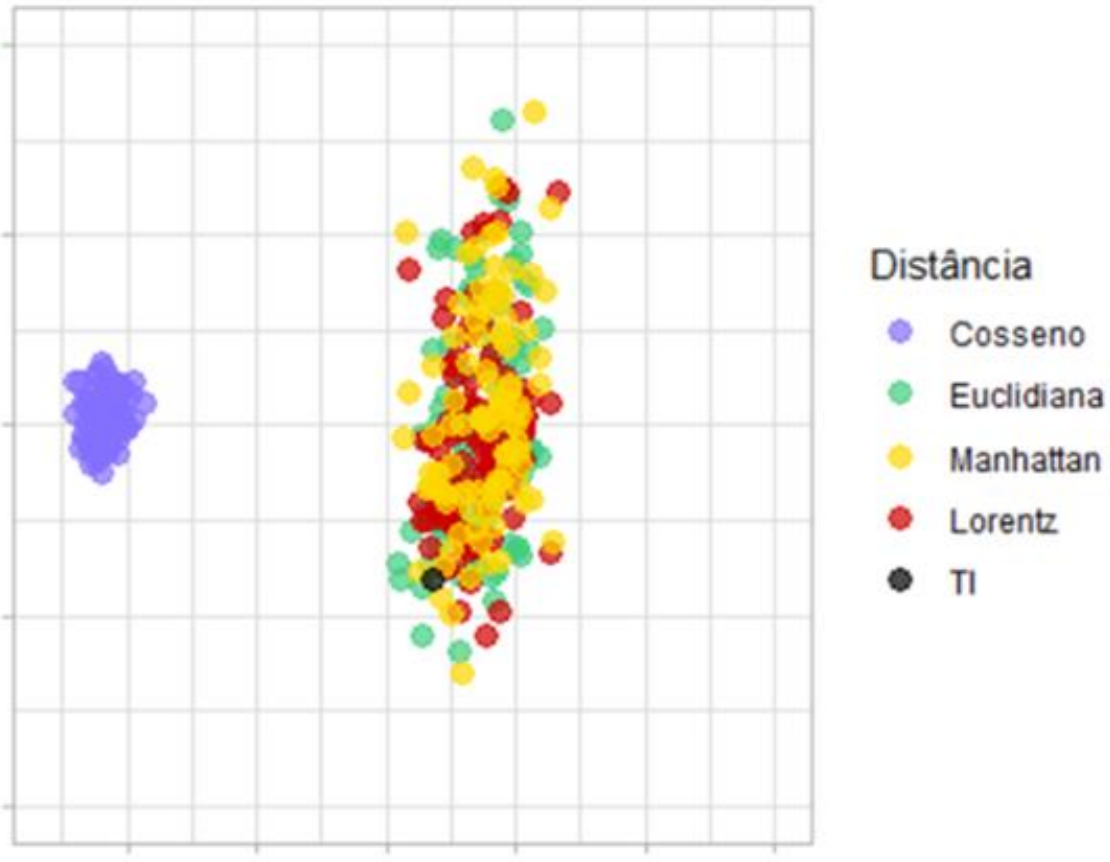

MDS da Média

b)

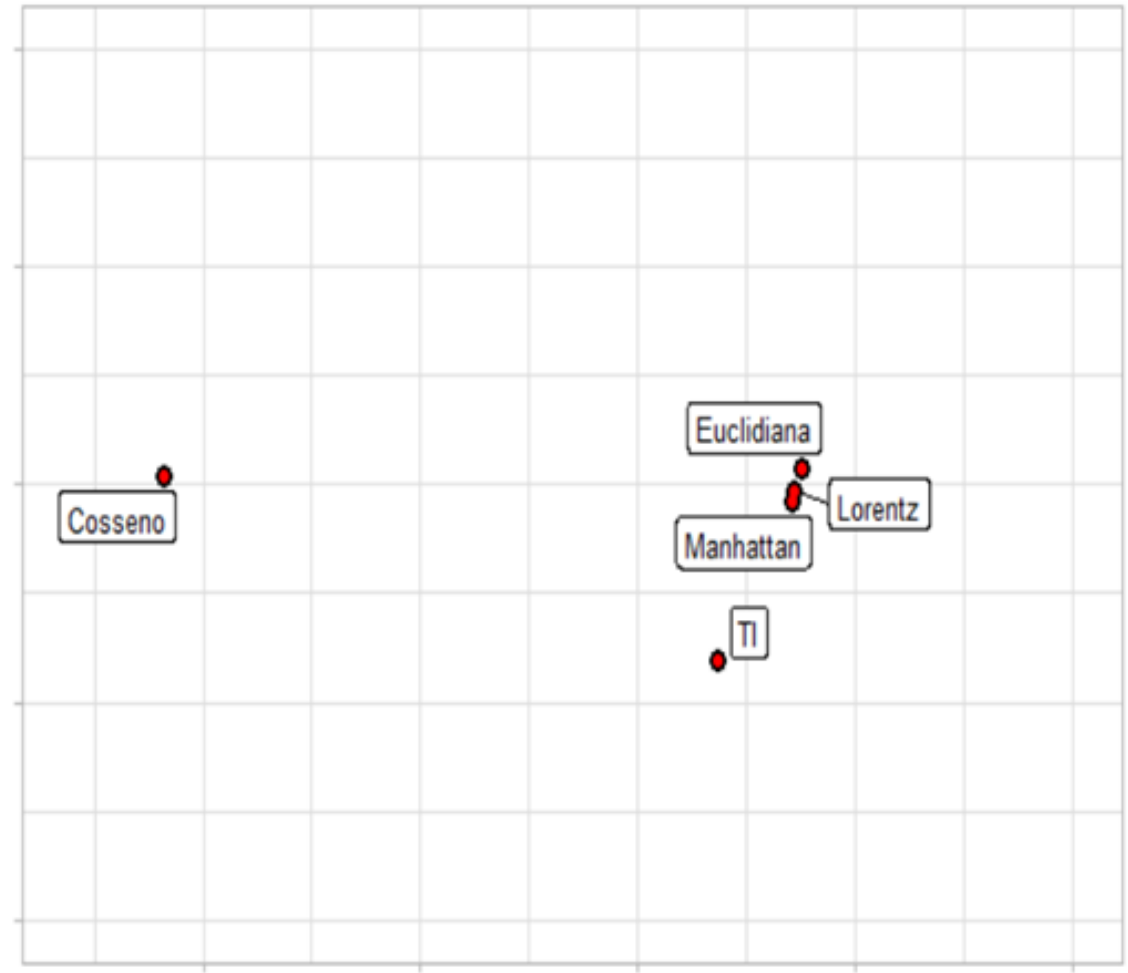

Figura 4.12 a) Representação do mapa de MDS, para demonstrar a variabilidade espacial de cada uma das simulações geradas pelo modelo binário $\mathbf{2 D}$. Como pode ser visto, a $\boldsymbol{D} \boldsymbol{C}$ é azul, a $\boldsymbol{D E}$ é de cor verde, a $\boldsymbol{D} \boldsymbol{L}$ tem cor vermelha e em amarelo a $\boldsymbol{D} \boldsymbol{M}$. Por último a $\boldsymbol{T I}$ é representada por um ponto de cor preta. $\boldsymbol{b})$ A média do MDS. 
Os resultados do MDS calculados para o $\boldsymbol{D B 2}$ (modelo de quatro categorias), são observados na Figura 4.13a. A $\boldsymbol{D} \boldsymbol{M}$ e a $\boldsymbol{D} \boldsymbol{L}$ apresentam melhor reprodução da continuidade, já que certa quantidade dos pontos se distribuem no entorno do ponto da $\boldsymbol{T I}$. Os resultados dessas duas distâncias aparentemente são mais adequados à reprodução dos padrões, de modo que, são consistentes com as realizações mostradas na Figura 4.3. Do mesmo modo que para o $\boldsymbol{D B 1}$, observa-se que as realizações obtidas com a $\boldsymbol{D E}$ os pontos estão mais dispersos no entorno do ponto da $\boldsymbol{T I}$, acredita-se que isso ocorreu, porque a reprodução da continuidade com a utilização dessa distância não é considerada adequada, uma vez que formou seus próprios padrões 0 que aumentou a variabilidade em relação à $\boldsymbol{T I}$. Finalmente, analisando-se os pontos do MDS obtidos para a $\boldsymbol{D C}$, vê-se que existem diferenças drásticas na reprodução dos canais, posto que todos os seus pontos se encontrem bem distantes da $\boldsymbol{T I}$. Na figura $4.13 \mathrm{~b}$ observa-se a media do MDS, onde pode ser verificado que a $\boldsymbol{D} \boldsymbol{L}$ e a $\boldsymbol{D} \boldsymbol{M}$ estão mais próxima a $\boldsymbol{T I}$. 


\section{MDS}

a)

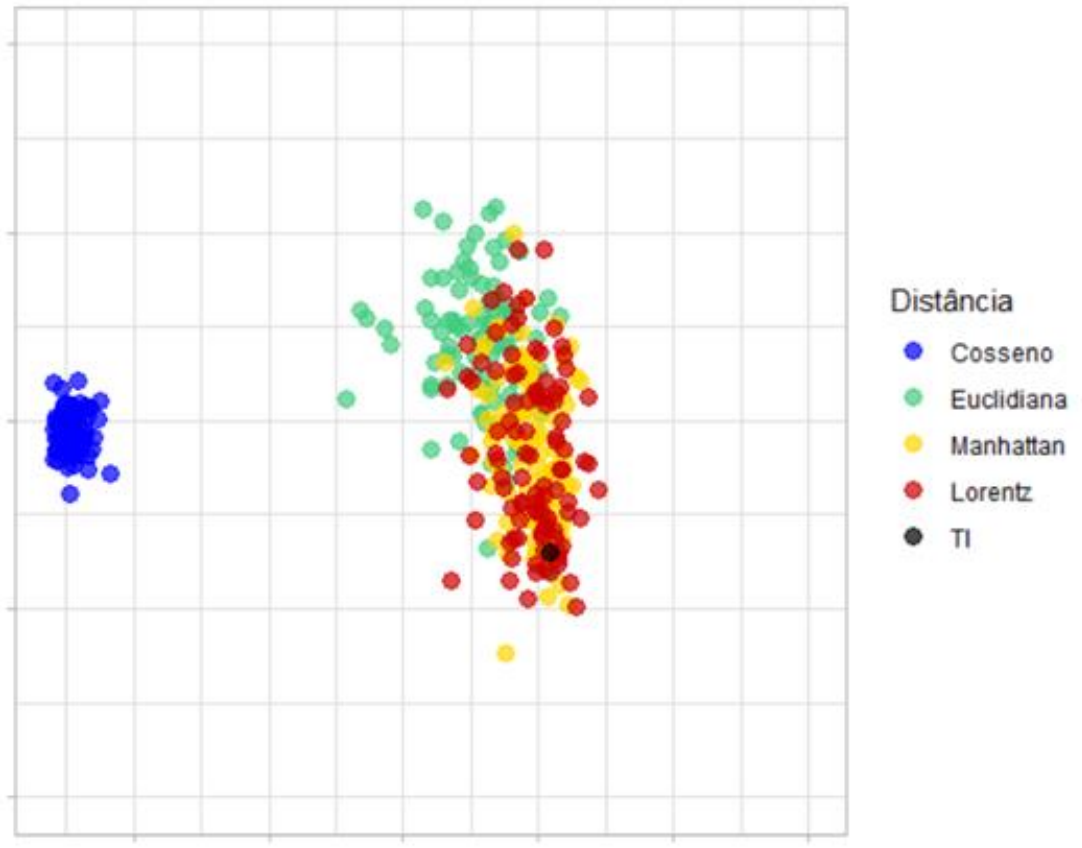

MDS da Média

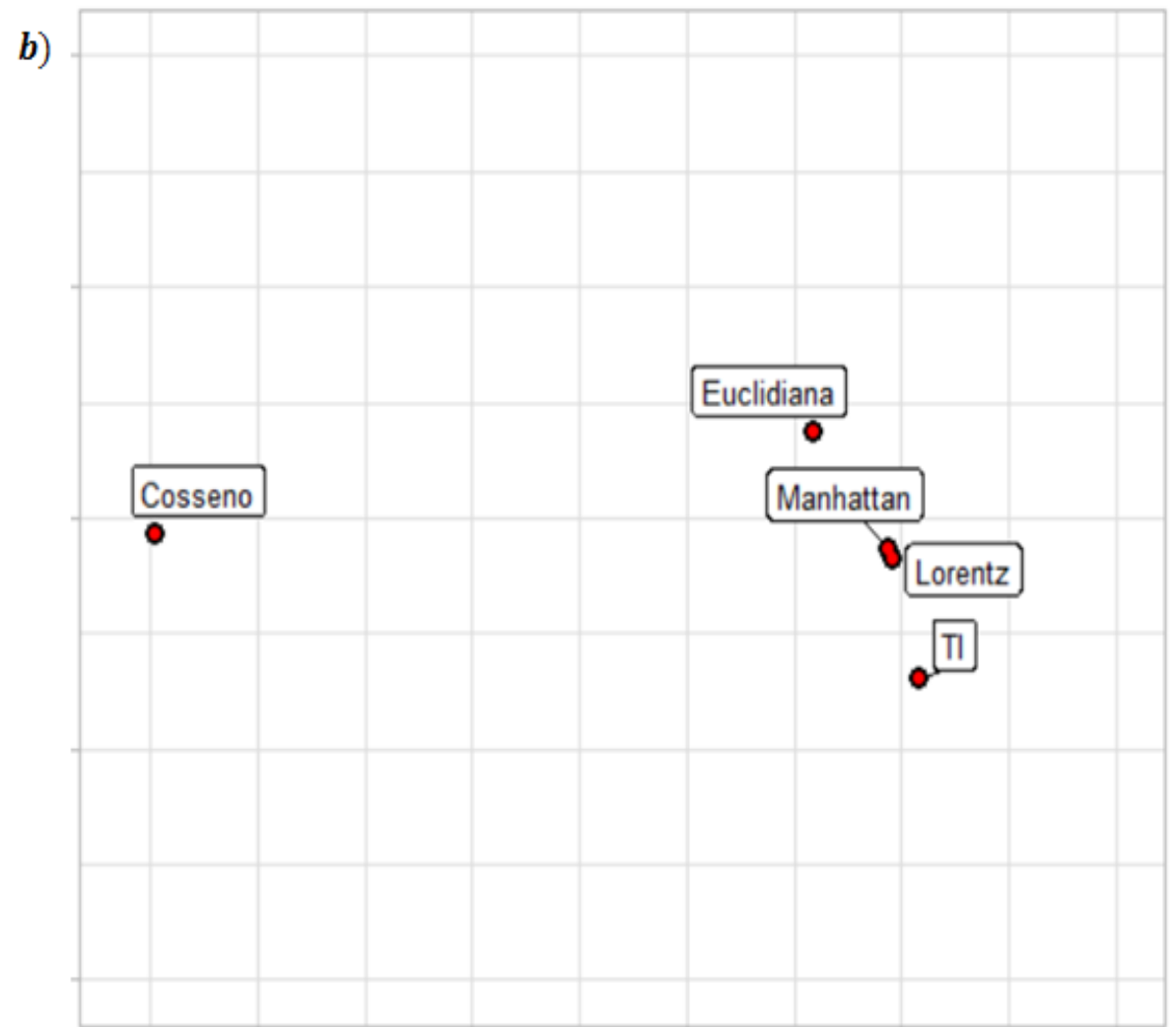

Figura 4.13 a) O gráfico MDS para demonstrar a variabilidade nas realizações geradas pelo $\boldsymbol{B D 2}$. $\boldsymbol{b}$ ) A média do MDS. 
Representa-se na Figura 4.14a o MDS das realizações do DB3 (muro de pedras), no qual se observa que uma determinada porcentagem de pontos da $\boldsymbol{D E}$ estão agrupados mais próximos da $\boldsymbol{T I}$, produzindo assim um menor espaço de incerteza e melhorando a reprodução dos padrões. Como pode ser visto a maioria dos pontos que representam à $\boldsymbol{D} \boldsymbol{L}$ e a $\boldsymbol{D} \boldsymbol{M}$ apresentam uma núvem de pontos dispersa em torno do ponto da $\boldsymbol{T I}$, mostrando que existe variabilidade elevada entre as realizações. Finalmente, os pontos que representam a nuvem de pontos da $\boldsymbol{D C}$, não capturam nenhuma das texturas da $\boldsymbol{T I}$, dado que todos os pontos estão afastados do ponto da TI. Observa-se na Figura $4.14 b$ que a $\boldsymbol{D} \boldsymbol{E}$ e a $\boldsymbol{D} \boldsymbol{L}$ estão em média mais próximos do ponto que representa a $\boldsymbol{T} \boldsymbol{I}$. 


\section{MDS}

a)

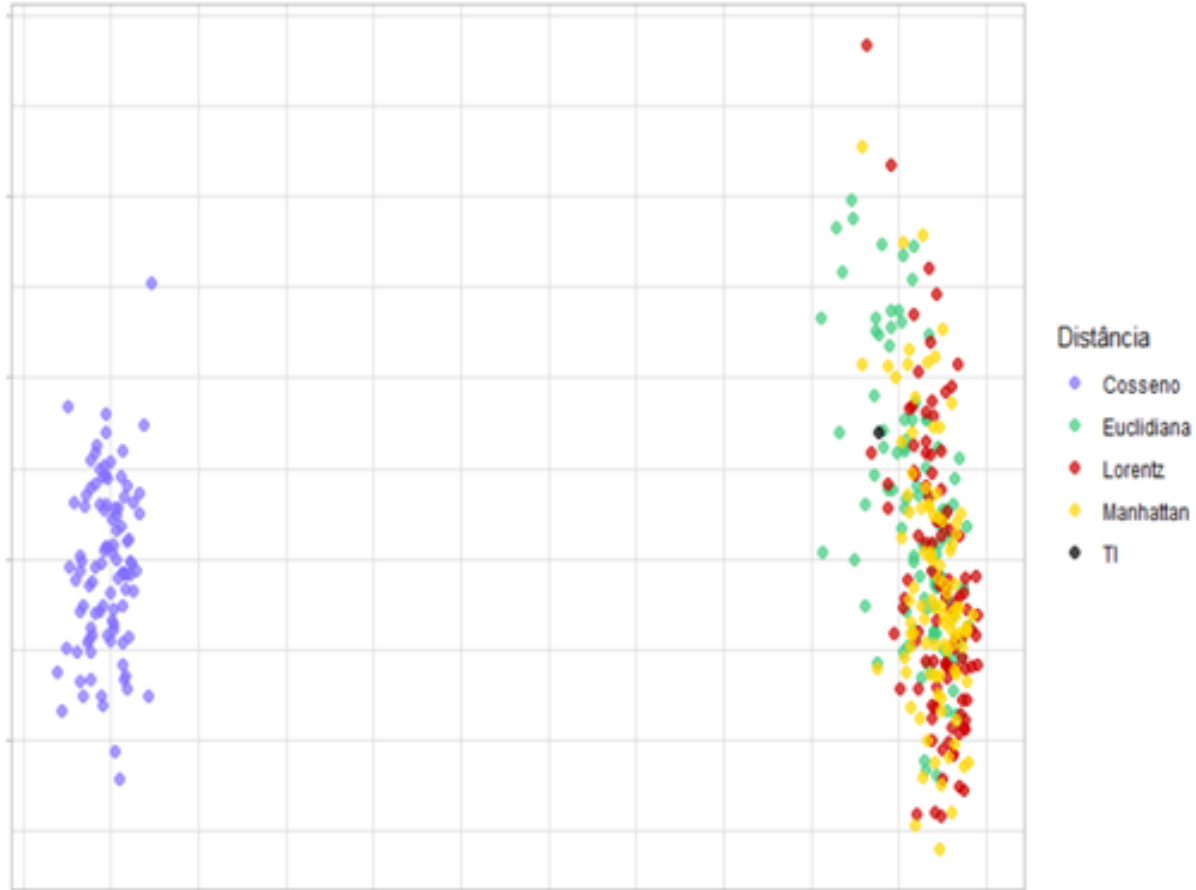

MDS da Média

b)

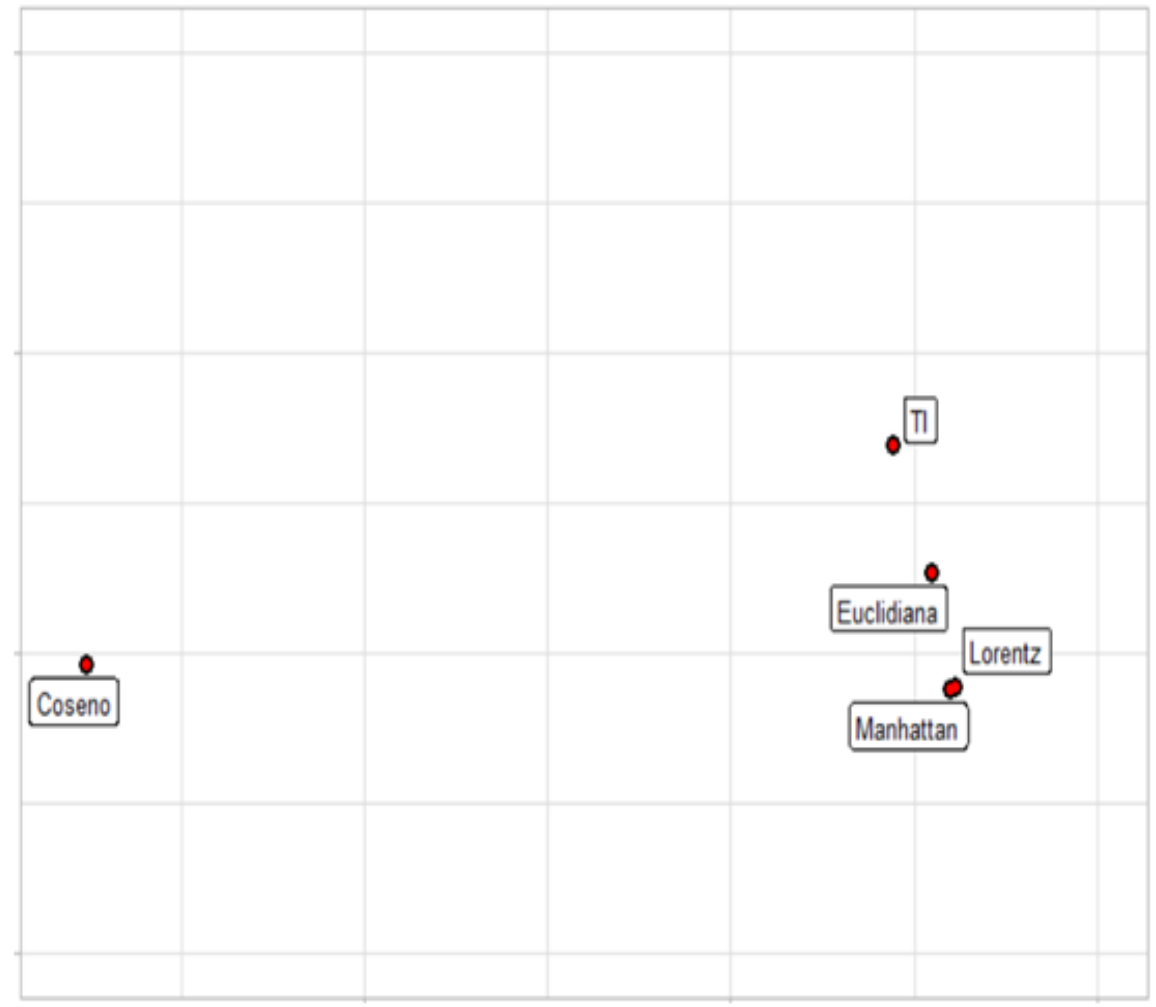

Figura 4.14 a) Representação do mapa MDS, para demonstrar a variabilidade espacial de cada uma das realizações geradas pelo modelo continuo. $\boldsymbol{b}$ ) A média do MDS. 
O gráfico de MDS, apresentado na Figura 4.15, foi elaborado a partir dos resultados obtidos das simulações do banco de dados $3 D$ (DB4). A Figura $4.15 a$ indica que as realizações da $\boldsymbol{D} \boldsymbol{L}$ distribuem-se preferencialmente no entorno do ponto da $\boldsymbol{T I}$. Os pontos amarelos e verdes correspondentes à $\boldsymbol{D M} \mathrm{e}$ à $\boldsymbol{D E}$ respectivamente, se encontram um pouco mais dispersos, que os pontos calculados a partir das distâncias apresentadas anteriormente, em função da $\boldsymbol{T I}$, representando um espaço de maior incerteza. O gráfico $4.15 b$ mostra-se que em media a distância que mais se aproximam da $\boldsymbol{T I}$ é a $\boldsymbol{D L}$. 


\section{MDS}

a)

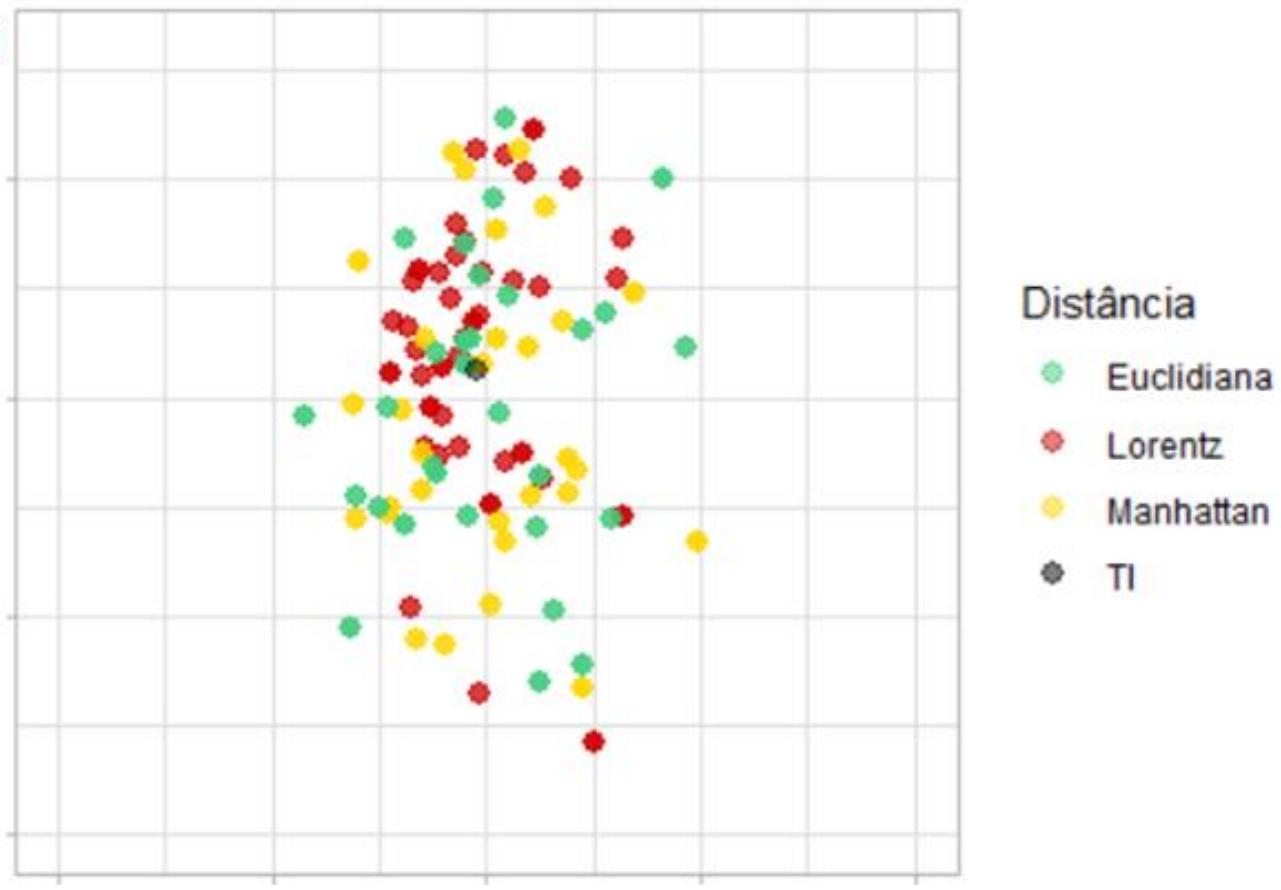

MDS da Média

b)

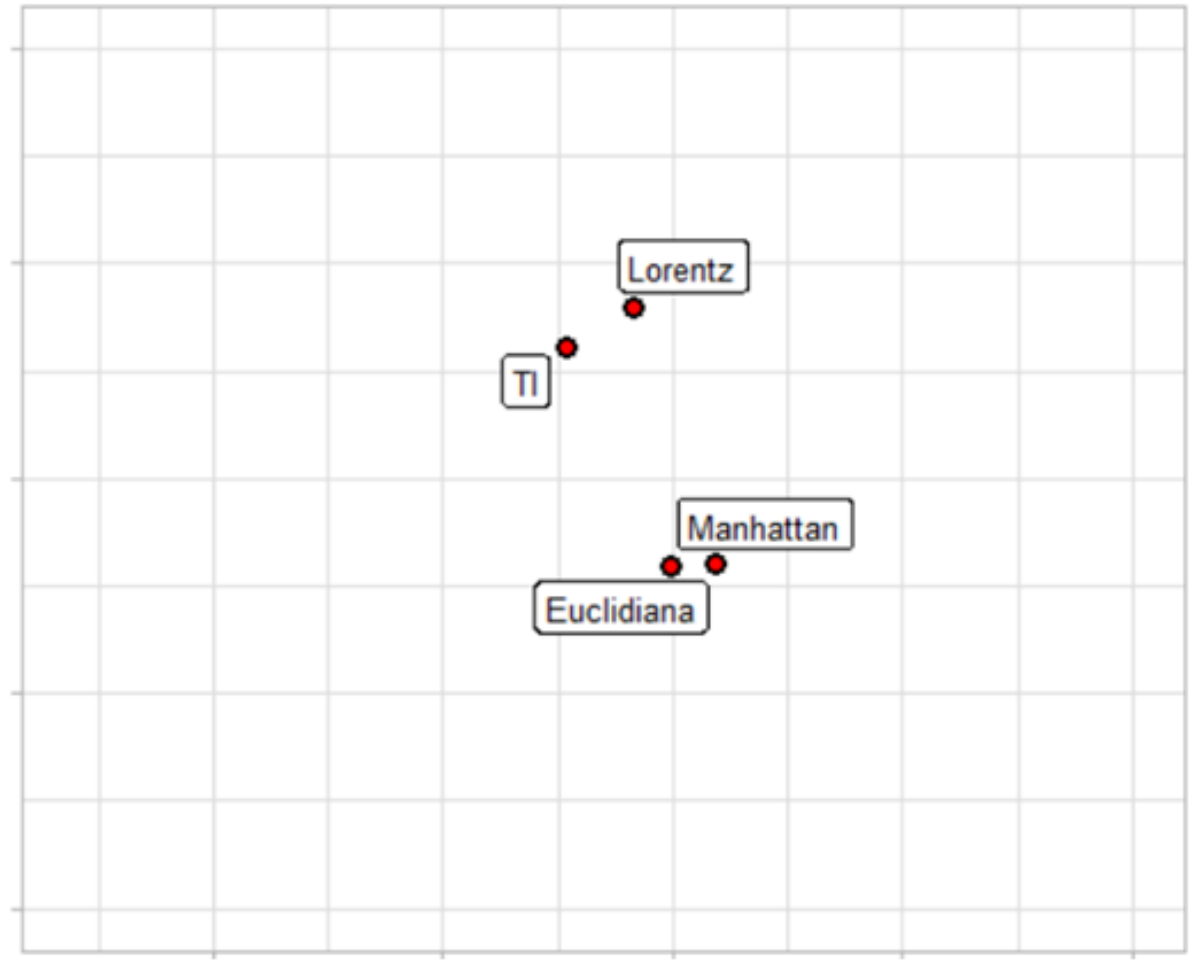

Figura 4.15 a) Um mapa MDS para demonstrar a variabilidade espacial de cada uma das realizações geradas pelo modelo binário $3 D$. b) A média do MDS. 


\section{CONCLUSÕES}

Após analisar a revisão bibliográfica no campo da simulação estocástica de múltiplos pontos, optou-se por criar, analisar e aplicar um novo algoritmo iterativo de MPS denominado SIMDISPAT, baseado no algoritmo SNESIM e aplicada no algoritmo SIMPAT. O desenvolvimento central do SIMDISPAT consistiu na utilização de amostragem aleatória simples, sobre a $\boldsymbol{T I}$, além da aplicação da tolerância da partição e da porcentagem do banco de dados. Apesar da seleção aleatória dos padrões e da diminuição percentual no banco de dados, os resultados não mostraram perda significativa na reprodução das características geológicas complexas nas diferentes $\boldsymbol{T I}$.

O desempenho do algoritmo, testado para os quatro bancos de dados sintéticos que foram selecionados como TI, mostrou-se adequado, pois o algoritmo condiciona bem os dados amostrados, além disso, o SIMDISPAT foi capaz de produzir com sucesso realizações condicionais de alta qualidade, tanto para as imagens categóricas, quanto para a contínua (muro de pedras).

Os resultados dos estudos de casos sintéticos evidenciaram que as variações dos parâmetros de entrada da seção 3.4 influenciaram positivamente na qualidade das simulações, obtendo uma boa continuidade espacial, onde os comportamentos foram capturados em maior e menor escala, tanto para o caso categórico quanto para o contínuo. A DC foi a distância que apresentou, sistematicamente, os resultados mais desfavoráveis na reprodução dos padrões.

A média (E-type) de cada um dos resultados das diferentes distâncias mostrou resultados adequados em reproduzir os padrões da TI para todas as distâncias, exceto a $\boldsymbol{D C}$.

Cabe destacar que o cálculo da entropia de Shannon é uma ferramenta muito útil, dado que o tamanho ótimo do template obtido, com certeza, melhora os resultados da MDS. 
A partir da análise de conectividade e da análise do MDS pôde-se verificar que as diferentes distâncias (Manhattan, Euclidiana e Lorentz) reproduzem alguns dos padrões das diferentes TI além de criar novos padrões. Pôde-se, ainda, concluir que os resultados das realizações da $\boldsymbol{D L}$, reproduzem satisfatoriamente todas as $\boldsymbol{T I}$ selecionadas, fato que pode ser explicado visto que o logaritmo natural é uma forma de reduzir o efeito dos erros. Embora com essa distância tenha havido aumento no tempo gasto de CPU a reprodução das continuidades melhorou. Por outro lado, a $\boldsymbol{D C}$ não apresentou nenhum resultado positivo, pois não conseguiu capturar nenhum padrão da TI. Pode ser devido ao fato de que o $\boldsymbol{T}$ 'ótimo' usado nas diferentes simulações era muito pequeno, já que com um $\boldsymbol{T}$ maior é possível capturar algumas características das imagens.

$\mathrm{Na}$ análise das médias dos pontos das nuvens de pontos do MDS que a distância de Lorentz e a distância de Manhattan apresentaram melhores resultados nos quatro estudos de caso realizados.

Finalmente o algoritmo proposto fornece uma boa qualidade na reprodução de padrões nas simulações de imagens geológicas, seja em $2 D$ ou $3 D$. 


\section{REFERENCIAS BIBLIOGRÁFICAS}

Allard, D. (1993). Simulating a Geological Lithofacies with Respect to Connectivity Information U sing the Truncated Gaussian Model. Geostatistical Simulations. Springer, 197-211.

Arpat, B. G. (2005). Sequential Simulation with Patterns. California, United States: Stanford University.

Arpat, G. B., \& Caers, J. (2007). Conditional Simulation with Patterns. Mathematical Geology, 39 (2), 27.

Bandyopadhyay, S., \& Saha, S. (1998). Unsupervised Classification. New York: Springer Science.

Bayer, P., Huggenberger, P., Renard, P., \& Comunian, A. (2011). Threedimensional high resolution fluvio-glacial aquifer analog: Part 1: Field study. J Hydrology, 405:1-9.

Boucher, A. (2009). Considering complex training images with search tree partitioning. Computers \& Geosciences, 35:1151-1158.

Boucher, A. (2011). Strategies for Modeling with Multiple-point Simulation Algorithms. Gussow Geoscience Conference, (pp. 1-9). Alberta.

Caers, J. (2011). Modeling Uncertainty in the Earth Sciences. John Wiley \& So.

Chatterjee, S., Roussos, D., \& Mustapha, H. (2012). Dimensional Reduction of Pattern-Based SimulationUsing Wavelet Analysis. Math Geosci, 44:343374.

Cuadras, M. (1996). The Multivariate Analysis Research Group. Spain: University of Barcelona.

Deutsch, C. V., \& Journel, A. G. (1992). GSLIB: Geoestatistical software library and user's guide. Oxford Unversity Press.

Deutsch, C. V., \& Wang, L. (1996). Hierarchical object-based stochastic modeling of fluvial reservoirs. Mathematical Geology, 28, 857-880.

Deutsch, C., \& Journel, A. (1998). Geostatistical Software Library and User's Guide. New York: Oxford University Press.

Goovaerts, P. (1997). Geostatistics for Natural Resources Evaluation. New York: Oxford University Press. 
Guardiano, F., \& Srivastava, M. (1993). Multivariate geostatistics: Beyond bivariate moments. Geostatistics Tróia, '92 , 1, 133-144.

Han, J., Kamber, M., \& pei, J. (2012). Data Mining Concepts and Techniques. USA: Elsevier.

Honarkhah, M. (2011). Stochastic Simulation of Patterns Using Distance-Based Pattern Modeling, PhD dissertation. Stanford University: Stanford, CA.

Honarkhah, M., \& Caers, J. (2010). Stochastich Simulation of Patterns Using Distance-Based Pattern Modeling. Mathematical Geosciences, 42, 487517.

Journel, A., \& Zhang, T. (2006). The Necessity of a Multiple-Point Prior Model. Mathematical Geology, 38 (5), 591-610.

Liu, Y., Gilbert, A., \& Journel, A. (2004). A Workflow For Multiple-Point Geostatistical Simulation. Springer, 1,245-254.

Liu, Y., Harding, A., Gilbert, R., \& Journel, A. (2004). A Workflow for Multiplepoint Geostatistical Simulation. Geostatistics Banff , 245-254.

Marie, M., \& Deza, E. (2009). Encyclopedia of Distances. London, New York: Springer .

Mariethoz, G., \& Caers, J. (2014). Multiple-point Geostatistics: Stochastic Modeling with Training Images. Chicester, United Kingdom: WileyBlackwell.

Mariethoz, G., Renard, P., \& Straubhaar, J. (2010). The direct sampling method to perform multiple-point geostatistical simulations. Water Resour Res 46.

Murty, M., \& Raghava, R. (2016). Support Vector Machines and Perceptrons. India: SpringerBriefs in Computer Science.

Mustapha, H., \& Dimitrakopoulos, R. (2010). High-order stochastic simulation of complex spatially distributed natural phenomena. Math Geosci, 42:457485.

Mustapha, H., Chatterjee, S., \& Dimitrakopoulos, R. (2014). Efficient Stochastic Simulation Through Decomposition of Cumulative Distribution Functions of Transformed Spatial Patterns. Mathematical Geosciences, 46: 95123.

Ortiz, J., \& Emery, X. (2004). Integrating Multiple-Point Statistics Into Sequential Simulation Algorithms. Geostatistics Banff, 969-978.

Panigrahi, N. (2014). Computing in Geographic Information Systems. London, New York: CRC Press.

Pardo-Igúzquiza, E., \& Dowd, P. (2003). CONNEC3D: a computer program for connectivity analysis of 3D random set models. Computers \& Geosciences, 775-785. 
Pyrcz, M., Boisvert, J., \& Deutsch, C. (2008). A library of training images for fluvial and deepwater reservoirs and associated code. Computers \& Geosciences, 34, 542-560.

Remy, N., Boucher, A., \& Wu, J. (2009). Applied Geostatistics with SGeMS: A User's Guide. Cambridge University Press: Cambridge.

Straubhaar, J., Renard, P., Mariethoz, G., Froidevaux, R., \& Besson, O. (2011). An improved parparallel multiple-point algorithm using a list approach. Mathematical Geosciences, 43, 305-328.

Straubhaar, J., Walgenwitz, A., \& Renard, P. (2013). Parallel multiple-point statistics algorithm based on list and tree structures. Math Geosci, 45:131-147.

Strebelle, S. (2000). Sequential simulation drawing structures from training images. Stanford Center for Reservoir Forecasting.

Strebelle, S. (2002). Conditional Simulation of Complex Geological Structures Using Multiple-Point Statistics . Mathematical Geology , 34, 21.

Strebelle, S., \& Cavelius, C. (2014). Solving Speed and Memory Issues in Multiple-PointStatistics Simulation Program SNESIM. Math Geosci, 46,171-186.

Strebelle, S., \& Remy, N. (2004). Post-processing of Multiple-point Geostatistical Models to Improve Reproduction of Training Patterns. Geostatistics Banff, 979-988.

Strebelle, S., \& Zhang, T. (2004). Non-Stationary Multiple-point Geostatistical Models. Geostatistics Banff, 235-244.

Sung-Hyuk, c. (2007). Comprehensive Survey on Distance/Similarity Measures between Probability Density Functions. International Journal of Mathematical Models and Methods in Applied Sciences, 1-8.

Tahmasebi, P. (2017). Structural adjustment for accurate conditioning in largescale subsurface systems. Adv Water Resour , 101.

Tahmasebi, P. (2018). Multiple Point Statistics: A Review. Handbook of Mathematical Geosciences, 613-643.

Tahmasebi, P., \& Sahimi, M. (2016a). Enhancing multiple-point geostatistical modeling: 2. Iterative simulation and multiple distance function. Water Resour Res, 52:2099-2122.

Tahmasebi, P., \& Sahimi, M. (2016b). Enhancing multiple-point geostatistical modeling: 1. Graph theory and pattern adjustment. Water Resour Res, 52:2074-2098.

Tahmasebi, P., Hezarkhani, A., \& Sahimi, M. (2012). Multiple-point Geostatistical Modeling Basedon the cross-correlation functions. Comput Geosci, 16, 779-797. 
Team, R. C. (2019). R: A language and environment for statistical computing. R Foundation for Statistical Computing, Vienna, Austria. URL https://www.R-project.org/.

Team, R. C. (2019). R: A language and environment for statistical computing. $R$ Foundation for Statistical Computing, Vienna, Austria. Retrieved from https://www.R-project.org/

Tran, T. (1994). Improving variogram reproduction on dense simulation grids. Computers \& Geosciences, 20, 1161-1168.

Wang, L., Yin, Y., \& Feng, W. (2018). A New Method to Select Training Images in Multi-Point Geostatistics. Scientific Research Publishing, 3, 112-129.

Zezula, p., Amato, G., Dohnal, v., \& Batko, M. (2006). Similarity Search The Metric Space Approach. United States of America: Springer.

Zhang, T., Switzer, P., \& Journel, A. (2006). Filter-Based Classification of Training Image. Mathematical Geology, 38 (1), 63-80. 


\section{APÊNDICE A - SIMDISPAT}

A principal fonte do algoritmo SIMDISPAT que é a entrada dos dados iniciais é chamada de instrução de parâmetros. Este arquivo permite a conexão entre funções do algoritmo.

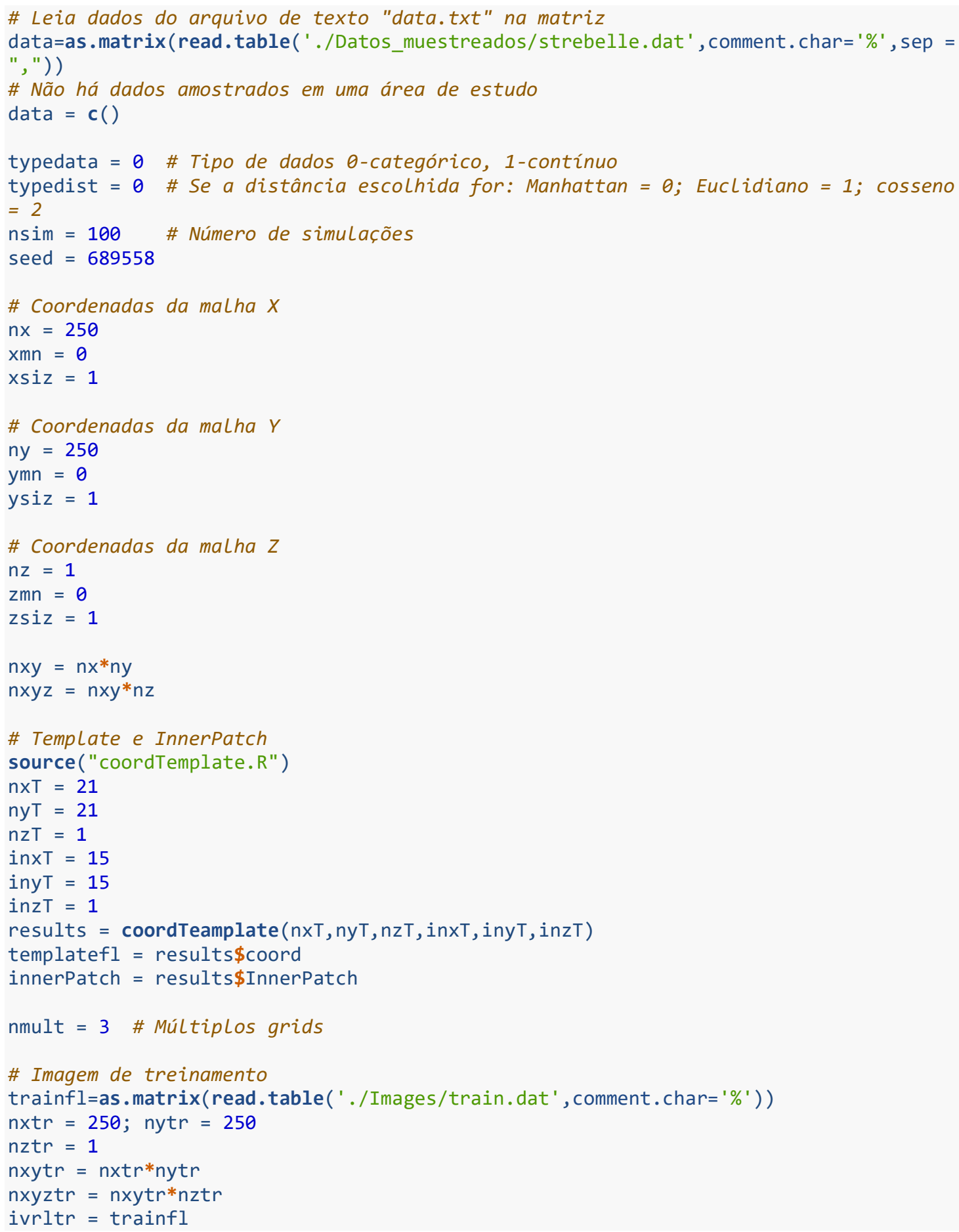




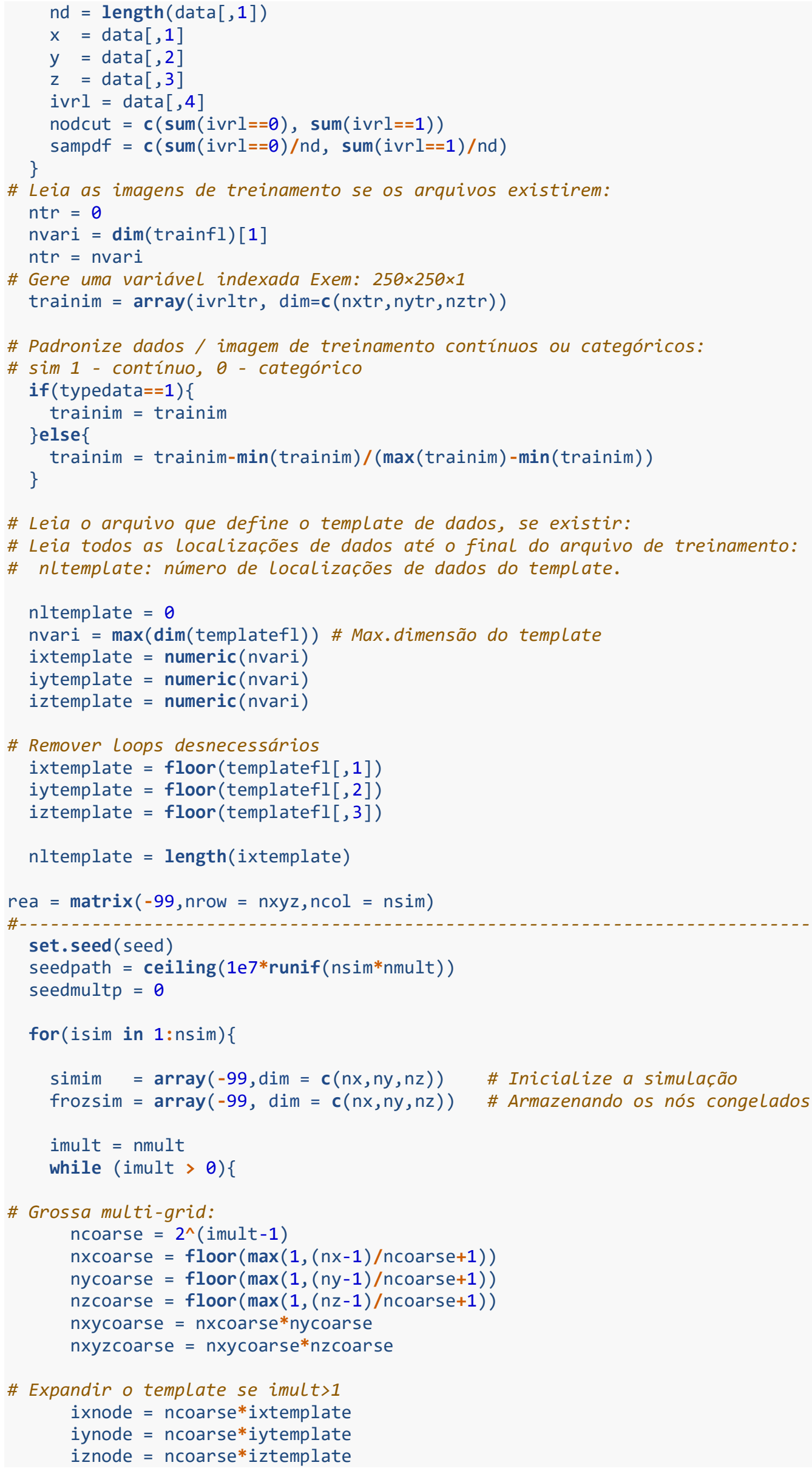


\# Construindo a base do padrão para cada correspondente multi-grid:

seedmultp $=$ seedmultp +1

results $=$ PatterBase(trainim, ixnode, iynode, iznode,

tp)

nltemplate, nxT, nyT, nzT, ncoarse, dbtol, patpercent, seedmul

patterns $=$ results $\$$ patterns

npdb = results\$npdb \# Número de padrões no banco de dados

\# Devolvendo os dados amostrados para cada correspondente multi-grid:

ncoarsest $=c$ (ncoarse, $n x c o a r s e$, nycoarse, $n z c o a r s e, n x y c o a r s e, n x y z c o a r s e)$

results $=$ AssignData $($ simim, frozsim, ncoarsest, $n d, x, y, z, i v r l$,

simim = results\$simim

xmn, ymn, zmn, xsiz, ysiz, zsiz)

frozsim = results\$frozsim

\# A função Ordepath gera um caminho aleatório que visita cada nó

\# apenas uma vez para cada tipo de malha, ou seja, malha grossa e fina.

idseed $=$ imult $+($ isim- 1$) *$ nmult

set.seed (seedpath [idseed])

orderpath $=$ sample $(\operatorname{seq}(1, n x y z c o a r s e)$, size $=$ nxyzcoarse, replace $=$ FALSE $)$

\# Executando a simulação para cada nó dentro do caminho aleatório: seedpattern $=$ seedpath[idseed]

simim = SimulationPath (simim, frozsim, patterns, npdb, ncoarsest, orderpath, ixnode, iynode, iznode, nltemplate, $\mathrm{nx}$, ny, $\mathrm{nz}$, innerPat

$\mathrm{ch}$, seedpattern, w1, w2, w3, dbtol)

imult $=$ imult -1

\} \}

rea $[$,isim $]=\operatorname{matrix}(\operatorname{simim}$, ncol $=1)$

$\operatorname{return}($ rea $)$

\}

\section{APÊNDICE B - coordtemplate}

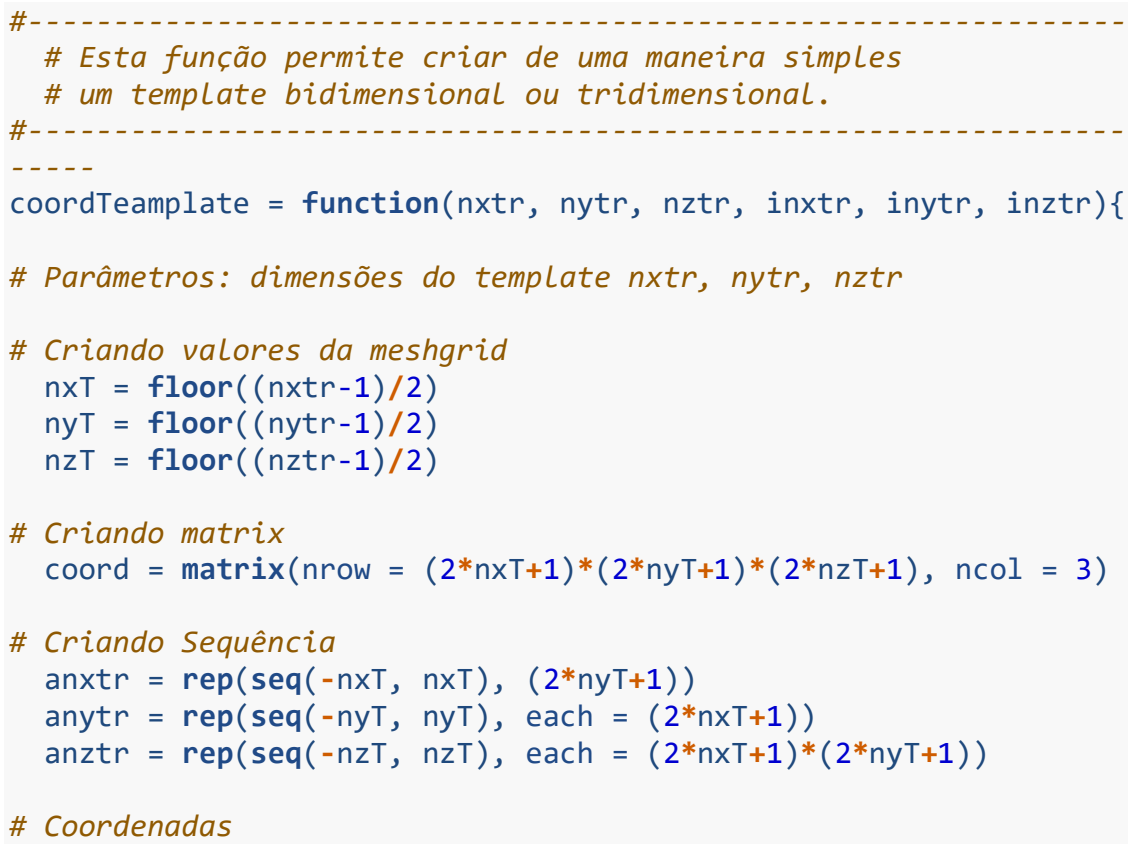




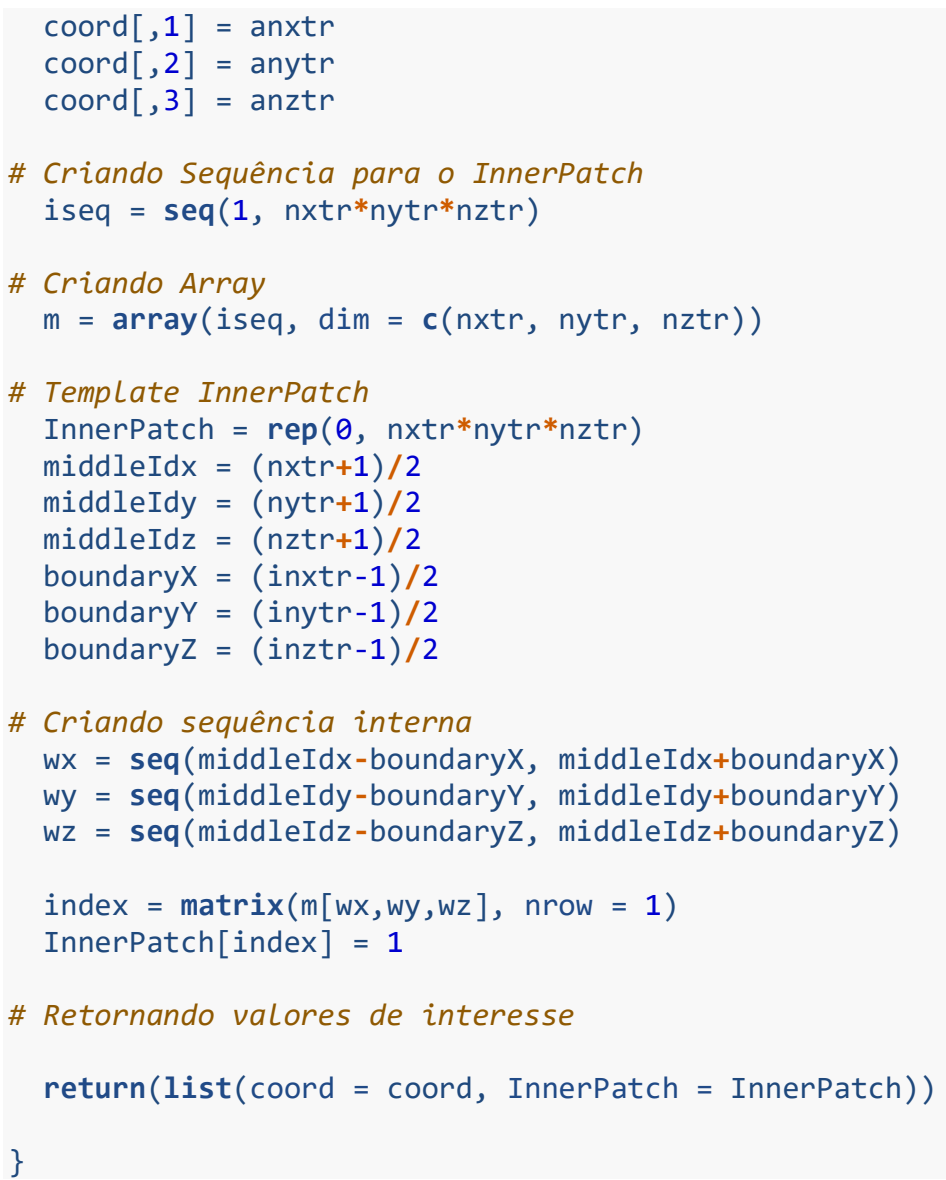

\section{APÊNDICE C - PatternBase}

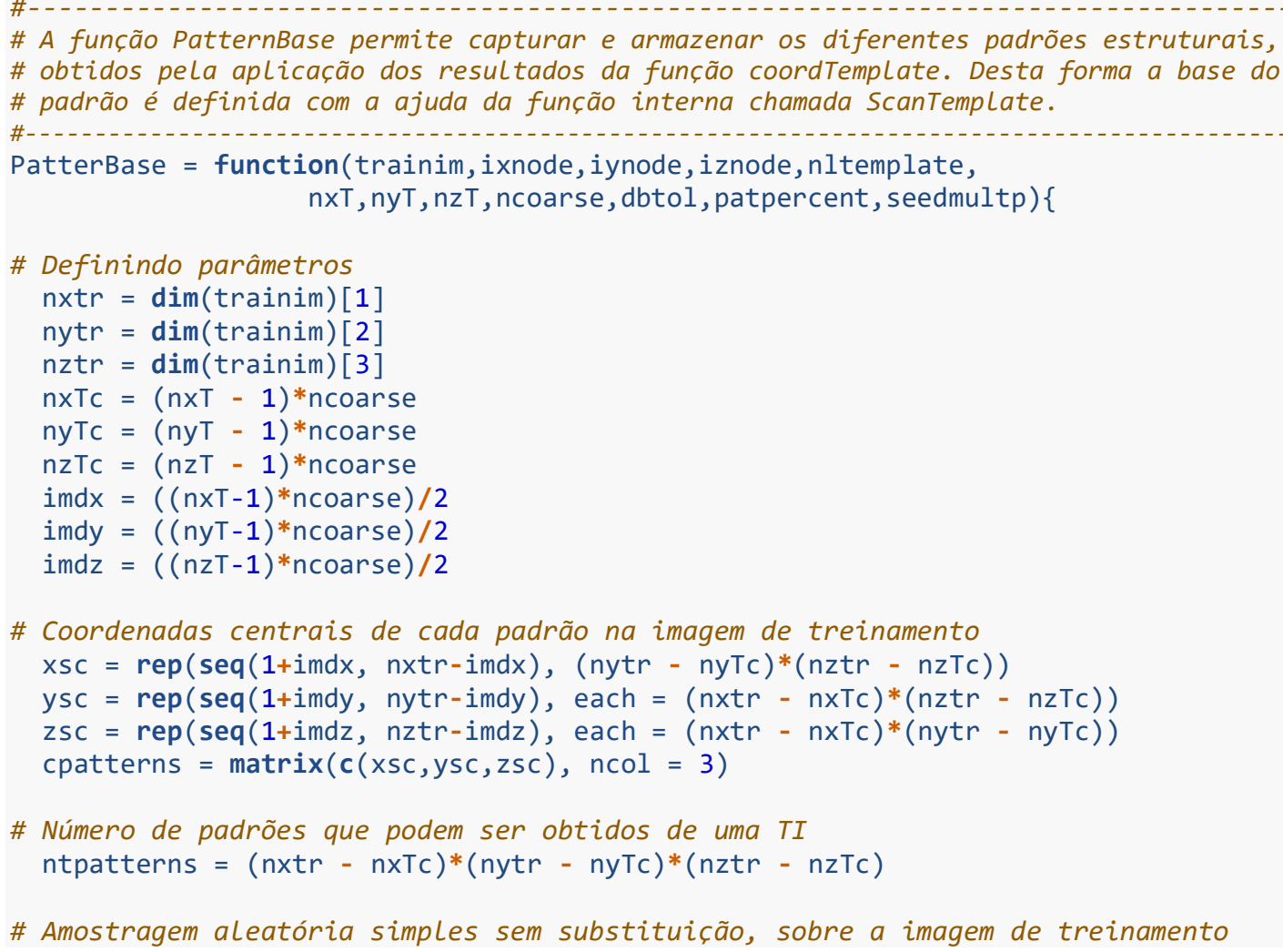




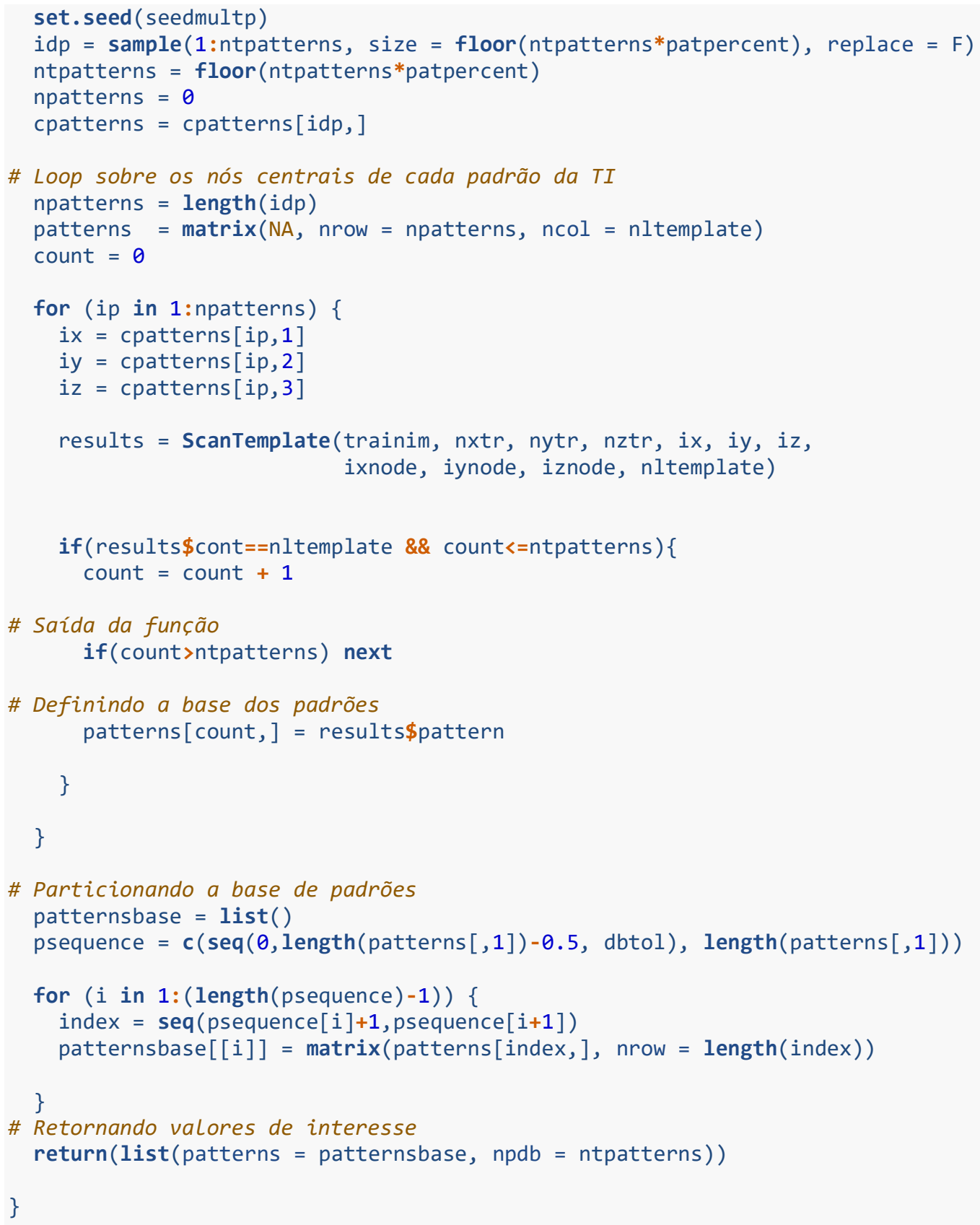

\section{APÊNDICE D - scantemplate}

\# Esta função verifica se as coordenadas do template estão completamente

\# dentro da imagem de treinamento.

ScanTemplate = function(trainim, nxtr, nytr, nztr, ix, iy, iz, ixnode, iynode, iznode, nltemplate) \{

\# Definição de coordenadas 


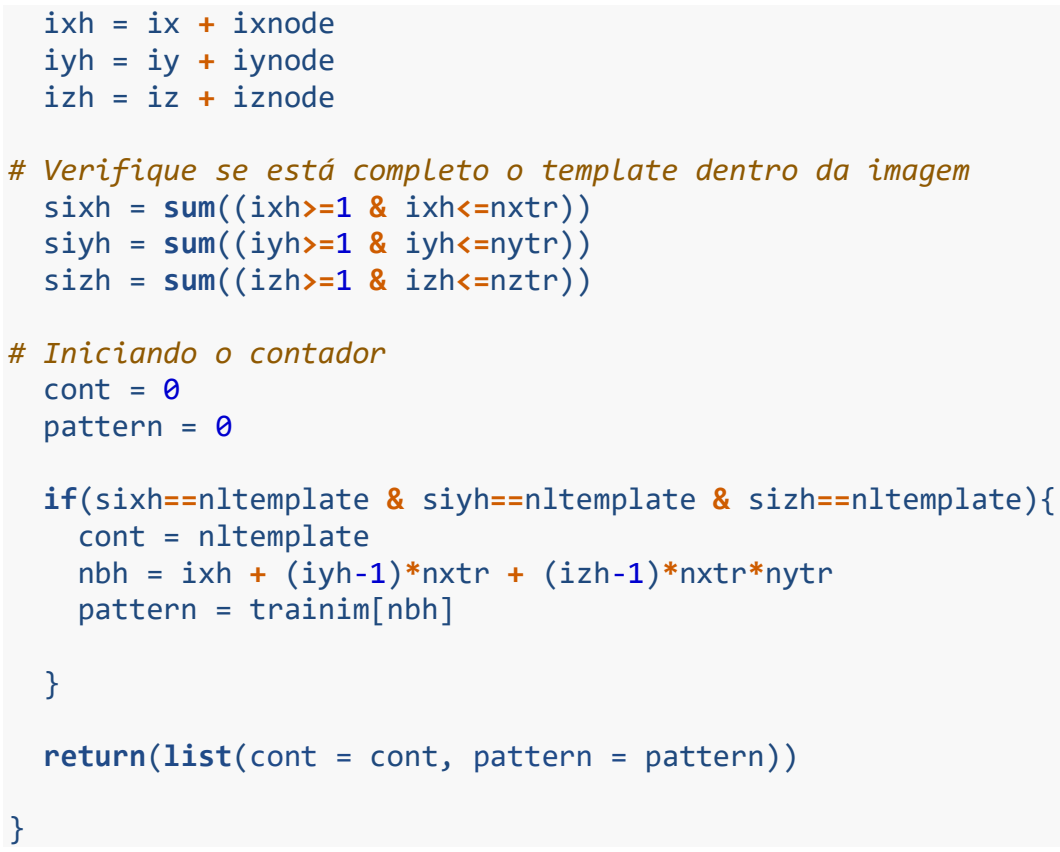

\section{APÊNDICE E - assigndata}

\# Esta sub-rotina atribui os dados de amostra originais aos nós

\# mais próximos da multi-grid que será simulada.

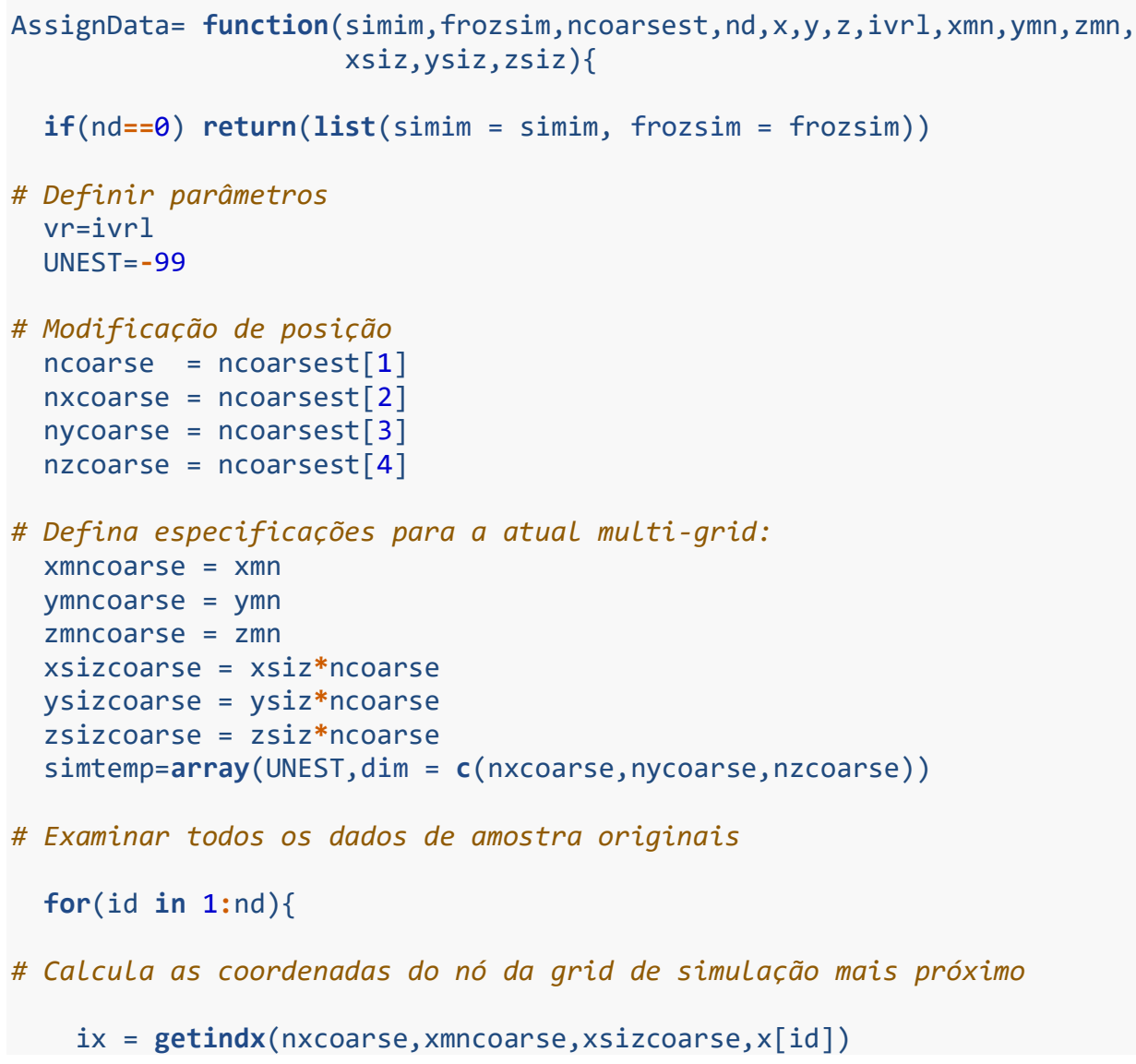




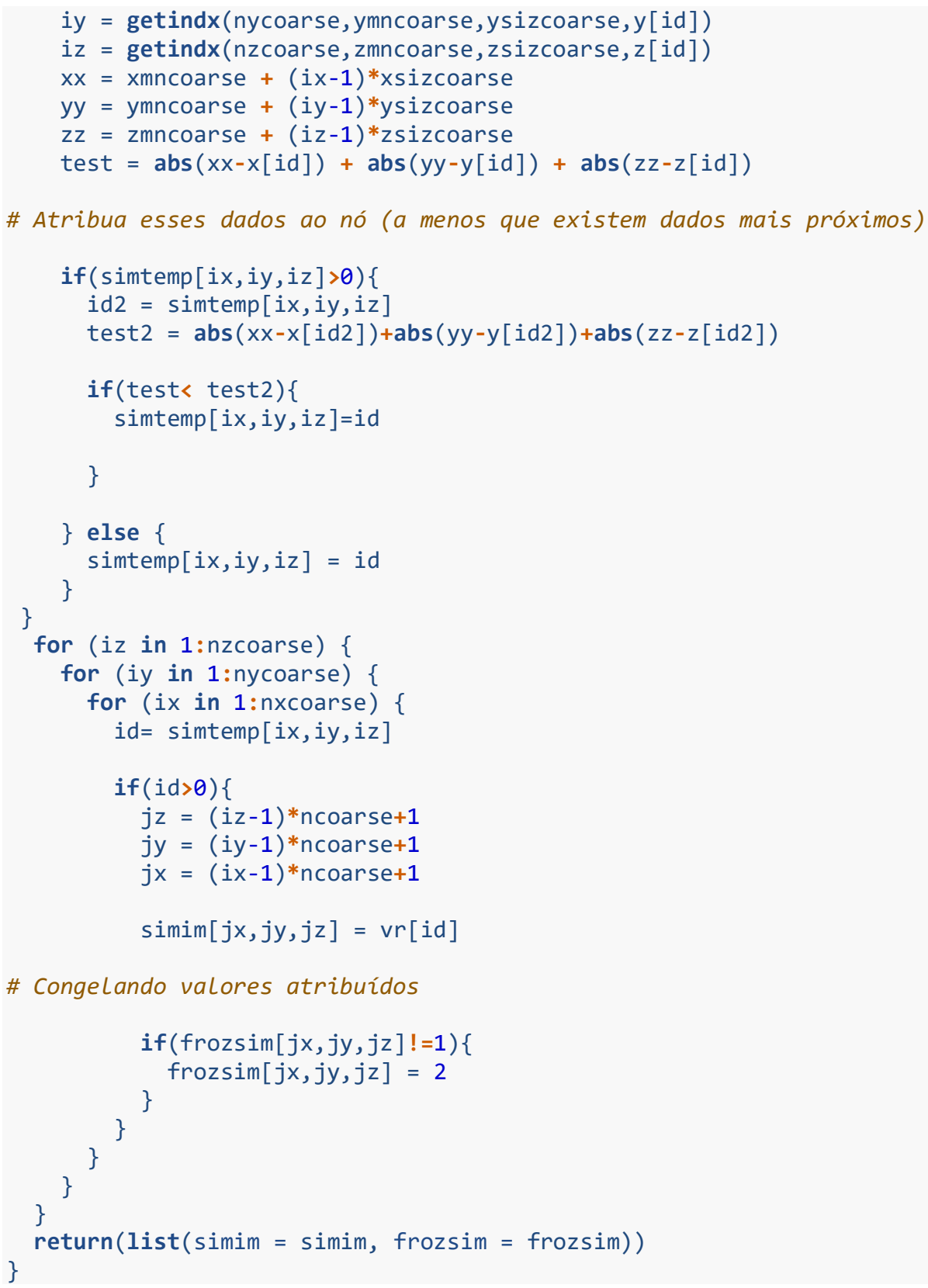

\section{APÊNDICE F - simulationpath}

\# É a função que percorre sequencialmente os nós da malha há serem

\# simulados de acordo com o caminho aleatório criado no Ordepath.

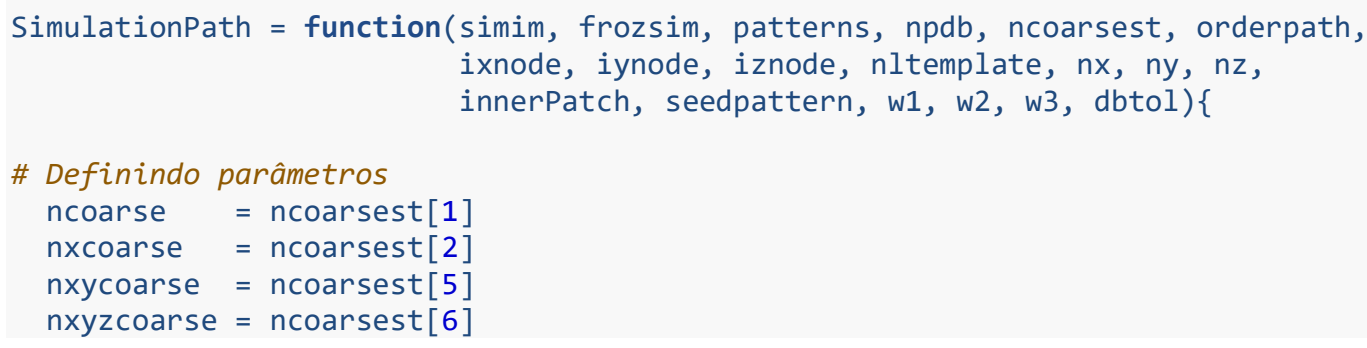




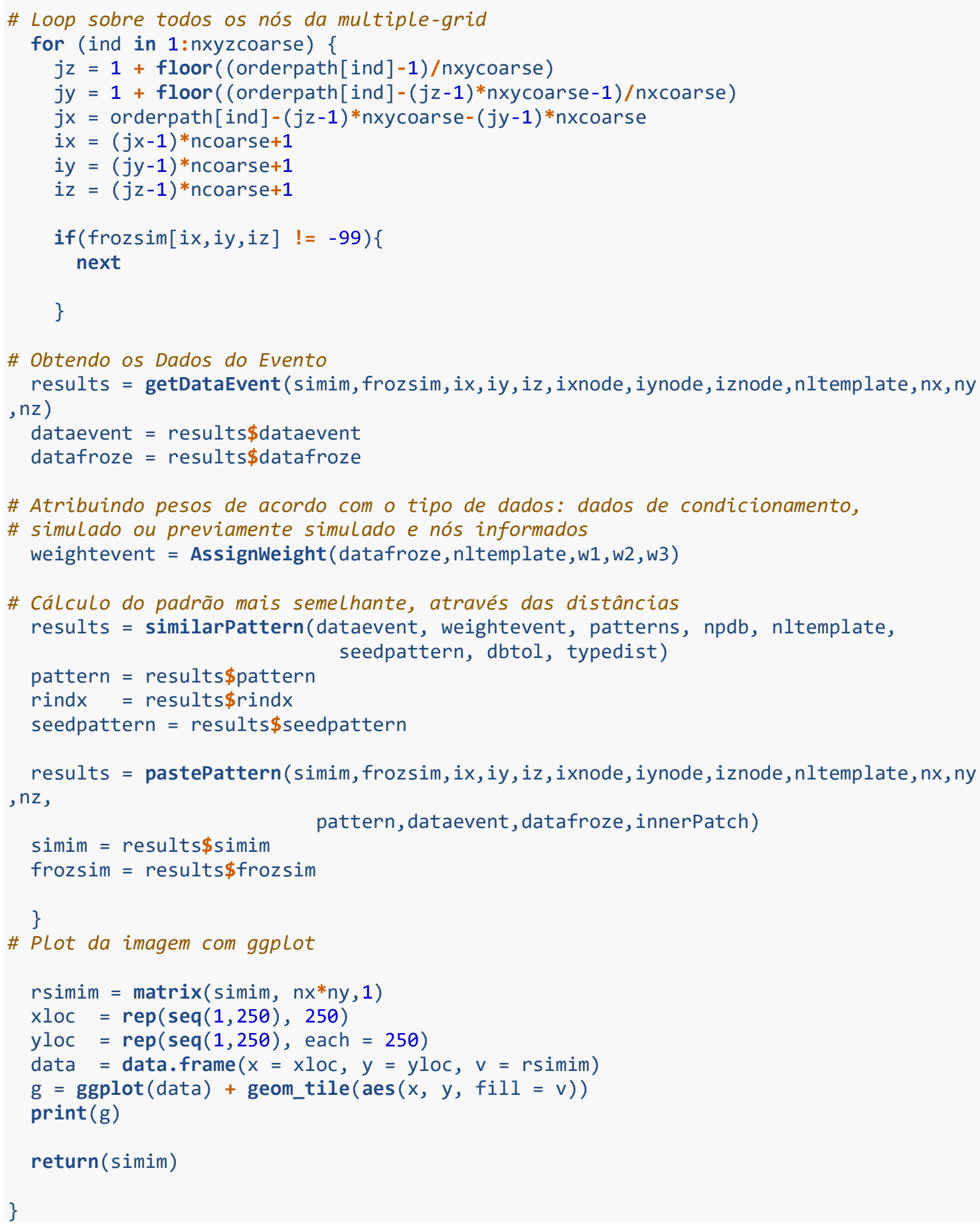

\section{APÊNDICE G - getdataevent}

\# É a função que mapeia os dados de condicionamento (dados brutos,

\# simulados anteriormente e outros)dentro do template, que esta centrado

\# no nó a ser simulados.

getDataEvent $=$ function $($ simim, frozsim, ix, iy, iz, ixnode, iynode, iznode, nltemplate, nx, ny, nz) \{

\# Definindo parâmetros 


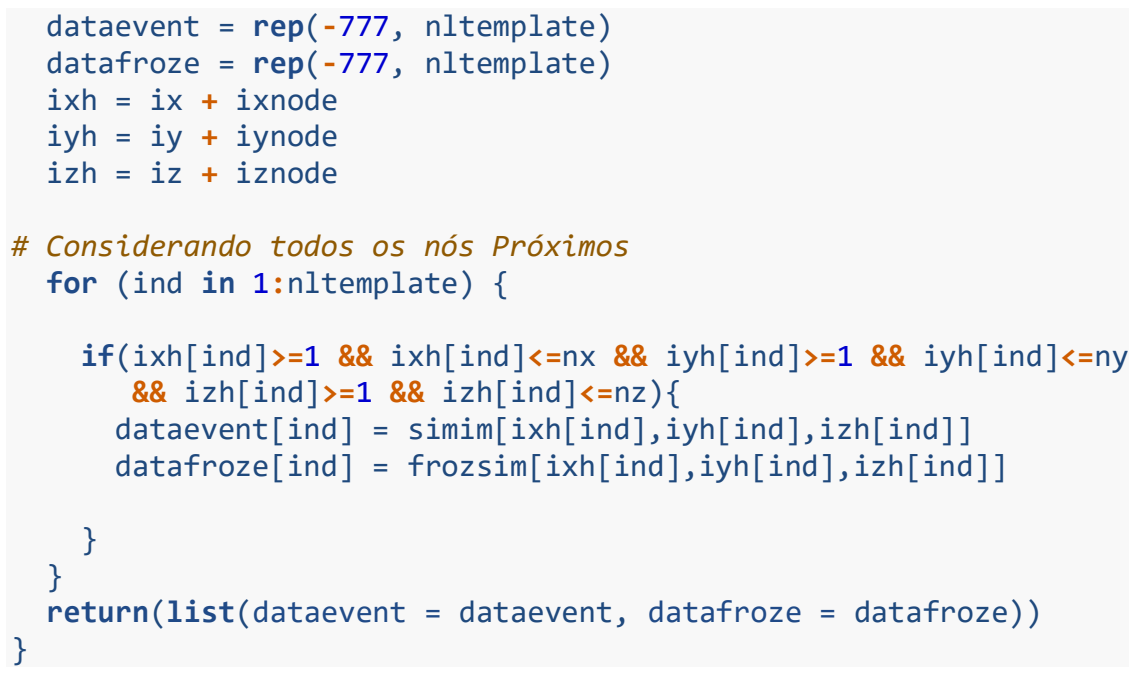

\section{APÊNDICE H - assignweight}

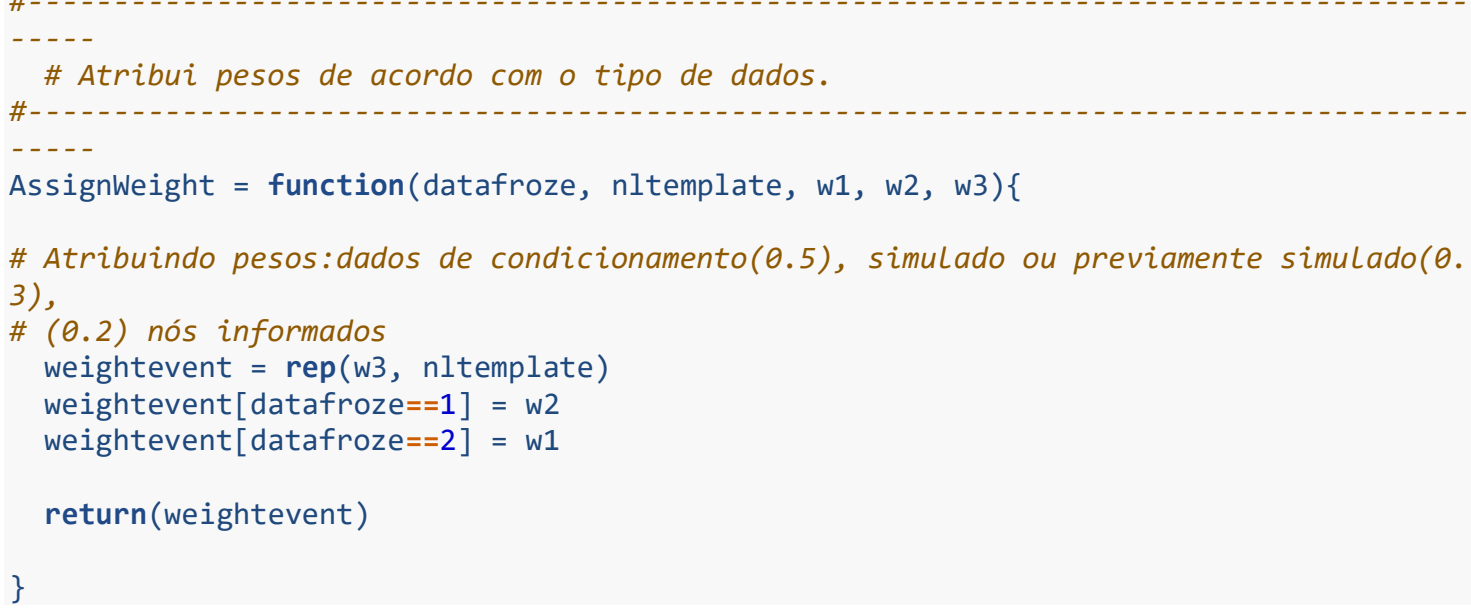

\section{APÊNDICE I - similarpattern}

\# Calculando o padrão mais semelhante utilizando uma das medidas de distancia.

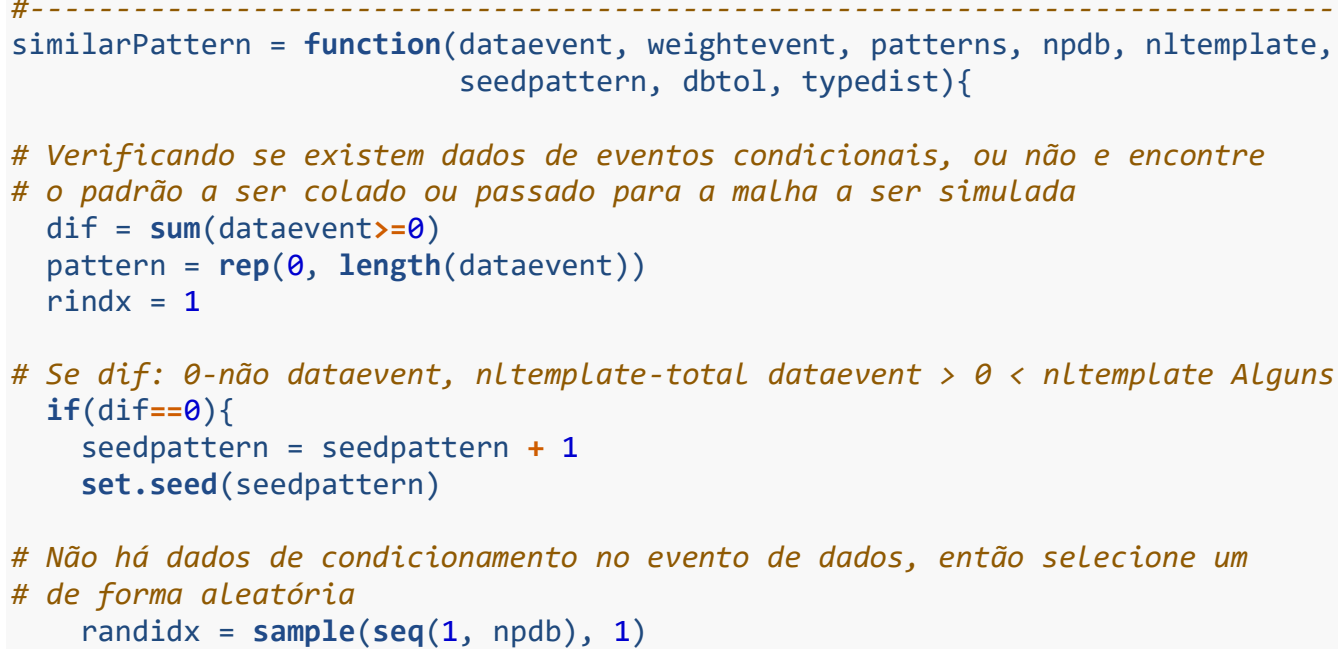




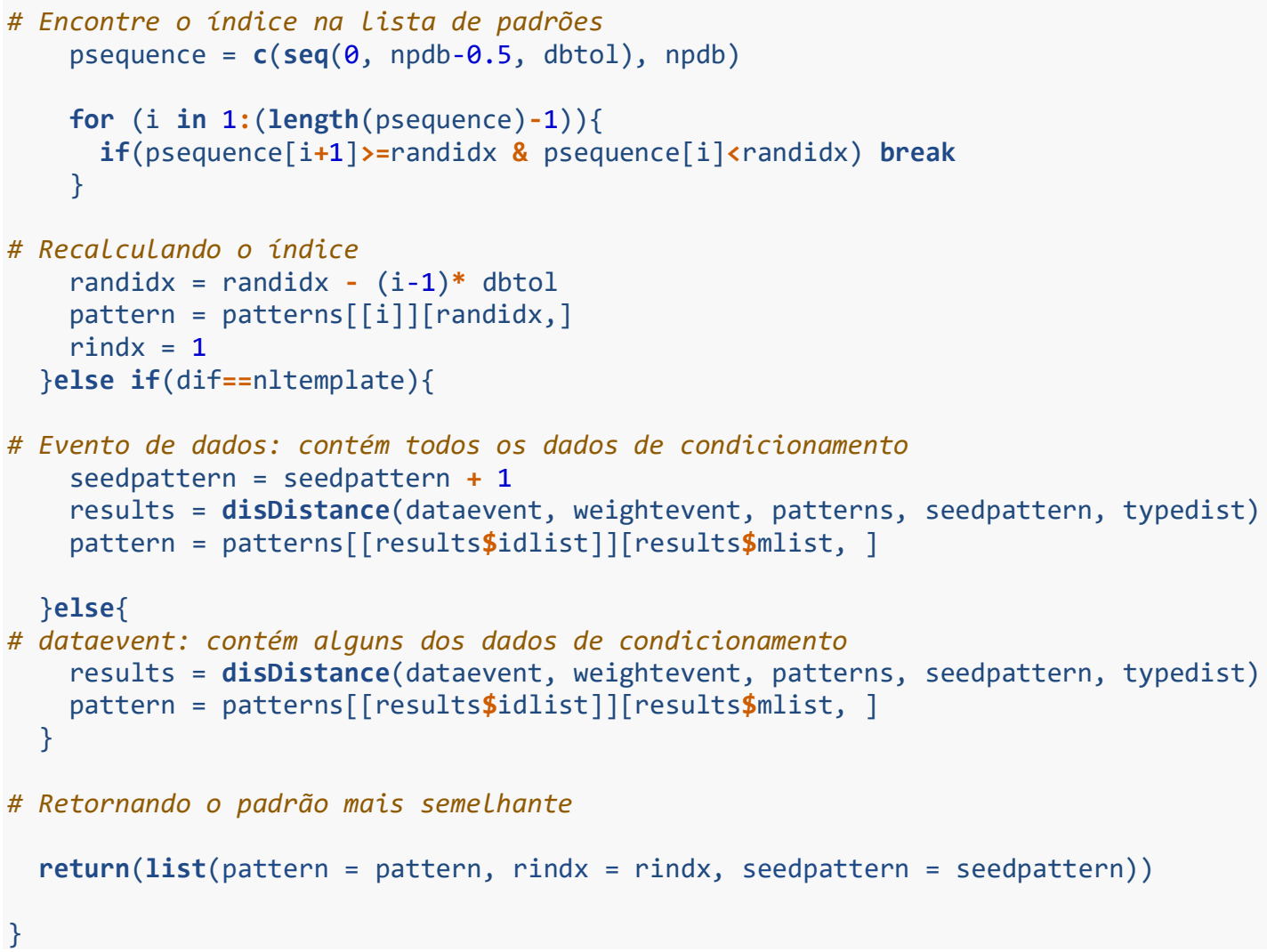

\section{APÊNDICE J - Disdistance}

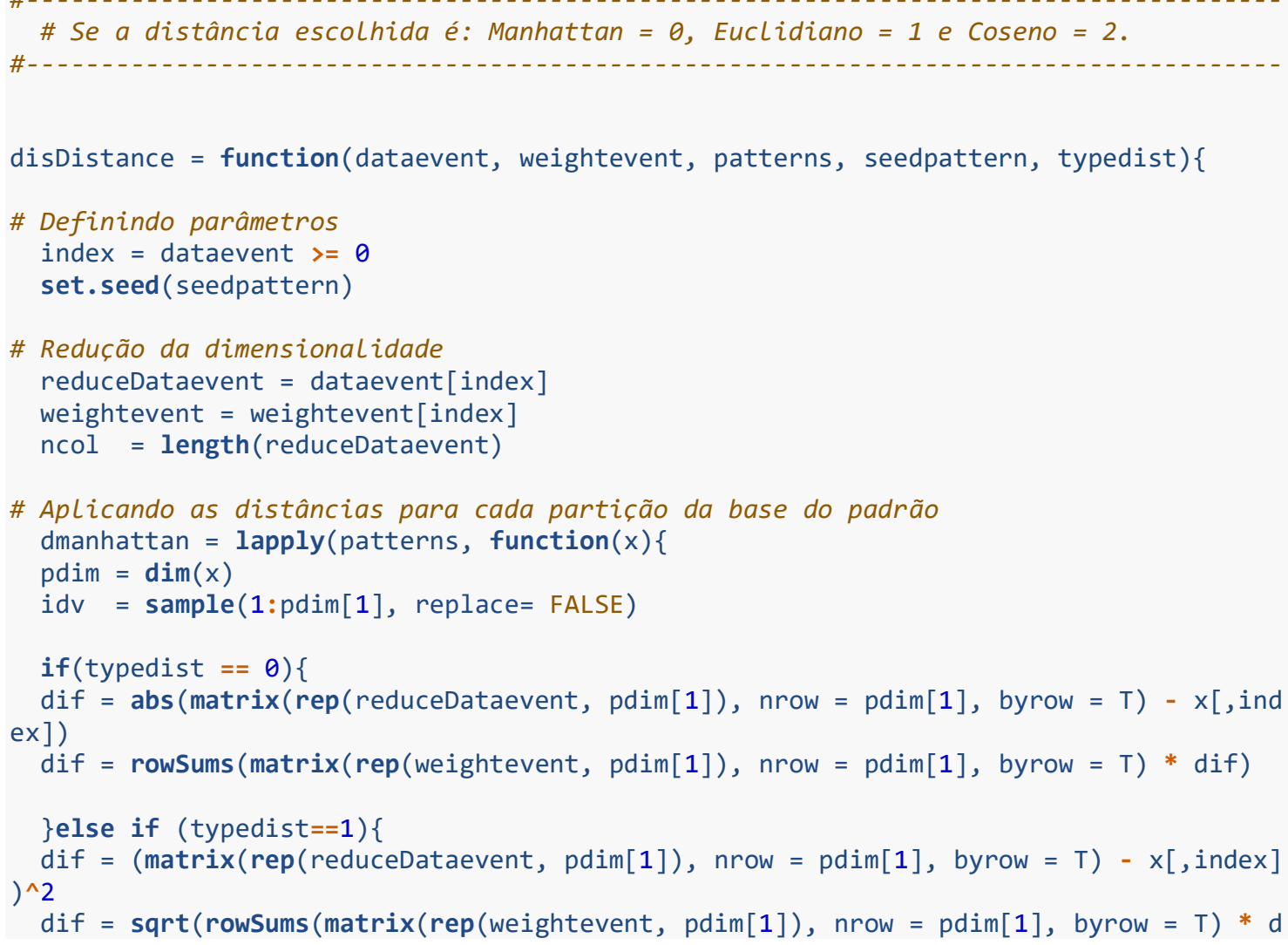


if))

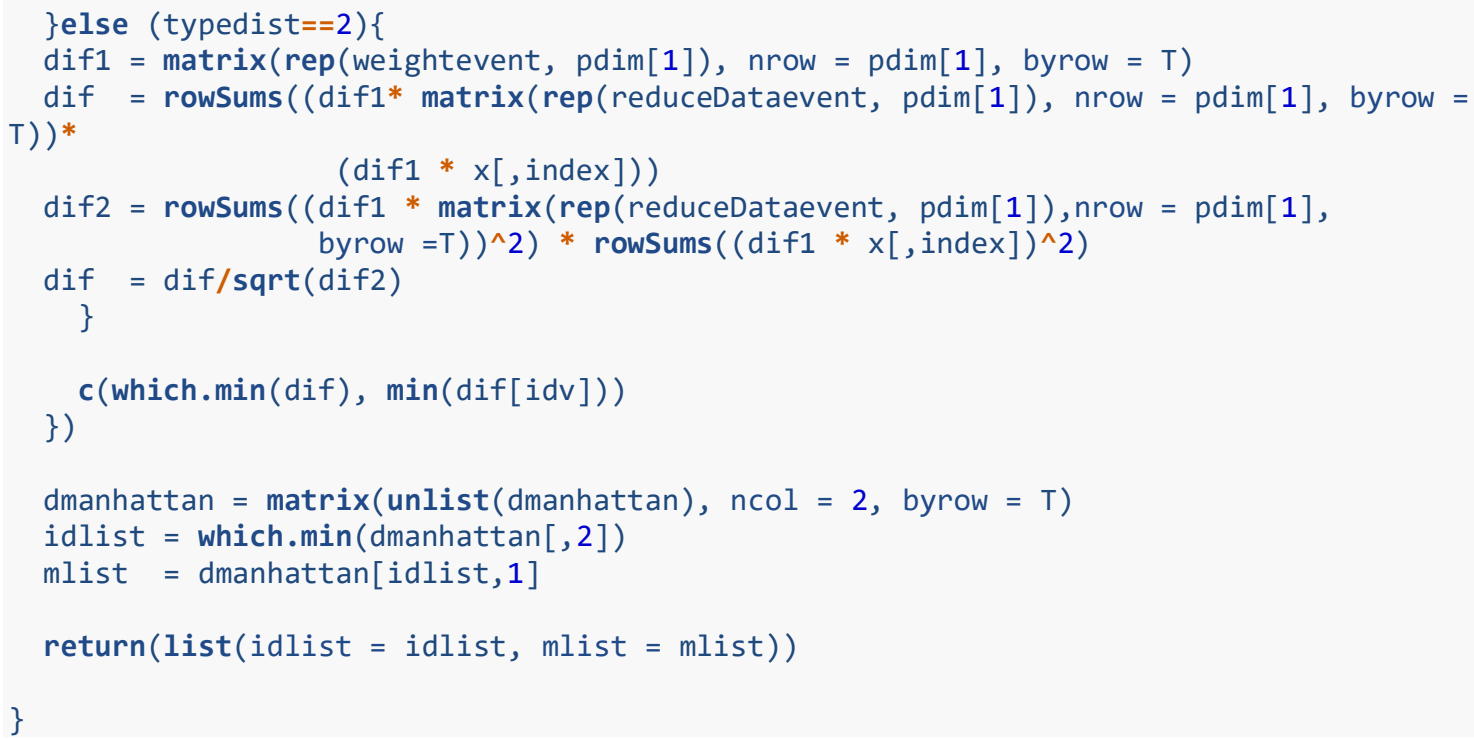

\section{APÊNDICE K - pastepattern}

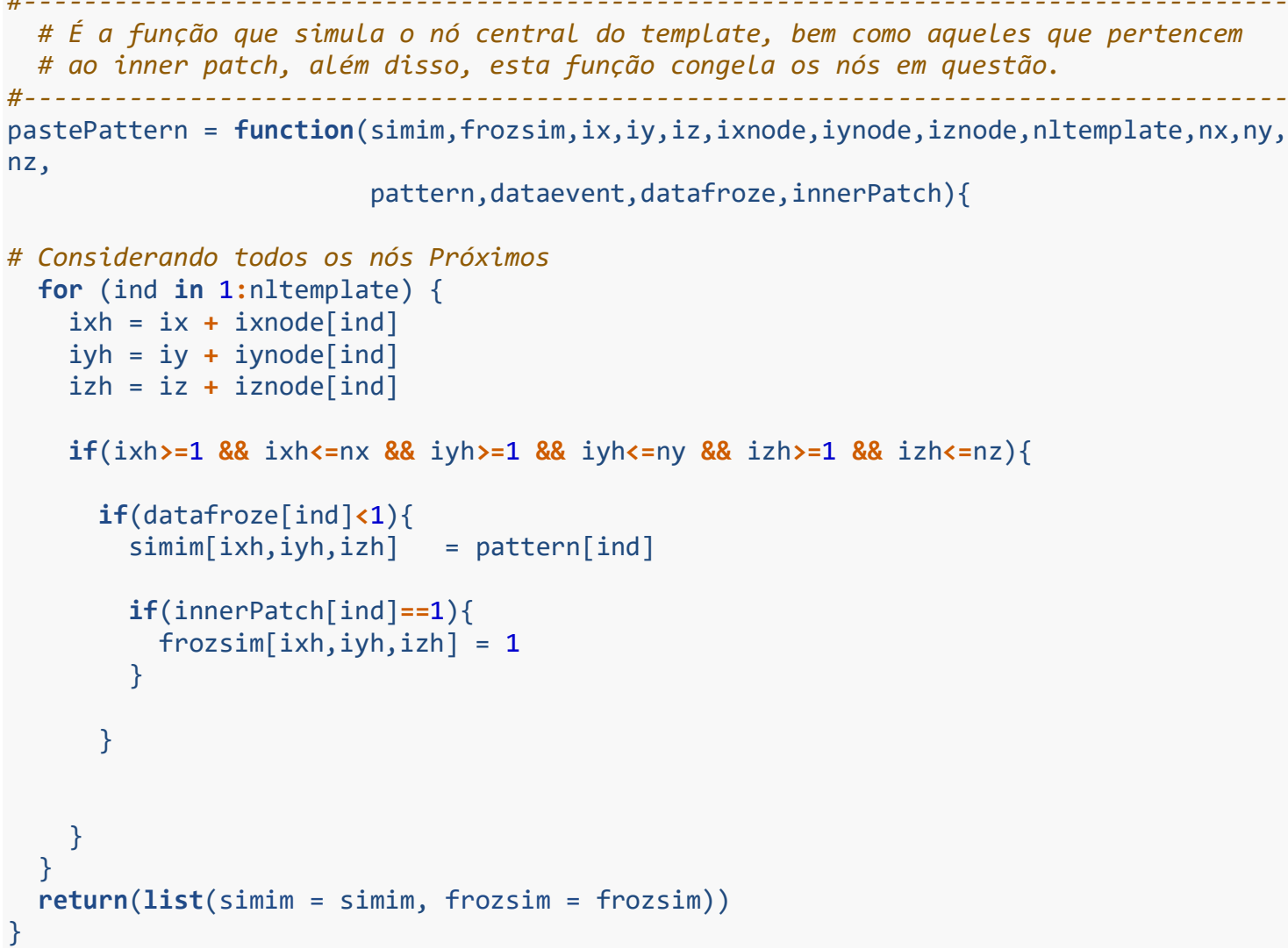

